\title{
Wetland flora and vegetation of the Western Australian wheatbelt
}

\author{
M.N. Lyons, N. Gibson, G.J. Keighery and S.D. Lyons \\ Department of Conservation and Land Management, Science Division, PO Box 51 \\ Wanneroo, Western Australia 6946, Australia
}

\begin{abstract}
Eight hundred and thirteen quadrats were surveyed to sample wetland vegetation throughout the wheatbelt and adjacent south coast of Western Australia, an area of ca. $220000 \mathrm{~km}^{2}$. Two hundred and fourteen wetlands were intensively sampled with between one and ten quadrats placed at each wetland to capture the major vegetation zonation. A further 100 quadrats were scattered through the study area to sample particular examples of wetland vegetation. Sampling spanned the full range of elevations at wetlands from inundated basins to the adjacent landforms such as lunettes.

A total of 1436 taxa of vascular plants from 109 families were recorded. Naturalized taxa were $11.8 \%$ of the flora. Four hundred and twenty-eight taxa $(29.7 \%)$ were categorized as restricted to wetland habitats. These included both aquatic ( 53 taxa) and amphibious plants ( 89 taxa). The wetlands of the study area represent the world centre of diversity for annual Juncaginaceae and the subfamily Salicomioideae (Chenopodiaceae).

Species richness of quadrats $\left(100 \mathrm{~m}^{2}\right)$ ranged from $1-72$ taxa, with a mean of 20.5 taxa per quadrat. Thirty-one per cent of taxa only occurred at a single quadrat (singletons) and $59.3 \%$ occurred at less than five quadrats. Eight previously unrecognized taxa were collected for the first time and the habitats and distributions of several undescribed taxa with few previous collections were clarified. Collections were made of 78 taxa listed on the Department of Conservation and Land Management's priority flora list. Eight of these were gazetted as Declared Rare Flora (Wildlife Conservation Act, 1950) with two (Frankeria conferta and F. paroula) regarded as extinct prior to the current survey,

Detailed cluster analysis was conducted on a reduced dataset ( 980 taxa by 760 quadrats) that excluded singleton species and some species-poor quadrats. A classification of 26 quadrat groups was defined. The primary division in the quadrat classification separated quadrats of inland largely naturally saline wetlands from quadrats at non-saline wetlands and at various wetland types with coastal and southwestern distributions. Lower levels in the classification were strongly related to local elevation and edaphic attributes of quadrats.

Thirty-four assemblages were defined from species cluster analysis and 29 were regarded as coherent species groups. The richness patterns of assemblages were modeled against 34 environmental attributes. Models explained between $26 \%$ and $74 \%$ of total deviance. Modeling was complicated by some assemblages having few members. Of the parameters used in the modeling, elevation at wetlands, substrate, electrical conductivity and pH were the dominant variables of models with the greatest decrease in deviance. Most assemblages were geographically well defined with annual precipitation and annual temperature range being important regional scalars.

Fifteen plant assemblages containing 472 native taxa are identified as being at particular risk from secondary salinisation. They include assemblages of both non-saline and naturally saline wetlands.
\end{abstract}

\section{INTRODUCTION}

The wetlands of the Southwest Botanical Province (SWBP) of Western Australia occur in an area recognised as one of 25 global biodiversity hotspots (Myers et al., 2000), largely because of the diversity of the flora. This diverse and highly endemic flora occurs in a landscape that is old, highly weathered and largely flat (Hopper et al., 1996; Beard et al., 2000).

The wheatbelt of Western Australia, within the SWBP, has seen some of the most rapid and extensive clearing of native vegetation on earth (Hobbs et al., 1993; Beresford et al., 2001). Mostly since the beginning of the $20^{\text {th }}$ century much of the 
native vegetation has been removed and replaced by annual crops and pasture (Hobbs et al., 1993). In the most cleared bioregion within the wheatbelt, the Avon Wheatbelt, over $90 \%$ of the native vegetation has been removed (Thackaway and Cresswell, 1995; Sheppard et al., 2002).

The dominant drainage systems of the wheatbelt are characterised by chains of salt lakes following the courses of Tertiary palaeo-rivers (Beard, 1999; 2000). These systems of salt lake chains extend well into the arid interior of Western Australia. Only along the western and southern margins of the ancient Yilgarn Craton has drainage been rejuvenated by marginal uplift during the Cretaceous (Beard, 1999). Drainage east of the Meckering line (Mulchay, 1967) and north of the Ravensthorpe Ramp (Cope, 1975) is effectively internal except in years of extreme rainfall.

Subdued drainage, coupled with the gradual deposition of salt from marine aerosols, has led to the slow accumulation of salt in wheatbelt soils and groundwater. Removal of native perennial vegetation altered the hydrological balance such that increased groundwater recharge and surface flow led to saline groundwater rise and the mobilization of salt stored in the soil profile (Teakle and Burvill, 1938). This secondary salinisation has had major impacts on wetlands and vegetation of valley floors (Wood, 1924; Teakle and Burvill, 1938; Froend et al., 1987; Halse et al., 1993; George et al., 1995). Predictions as to the area of wheatbelt shires that will eventually be impacted by shallow watertables range from between $22 \%$ and $34 \%$ (Short and McConnel, 2001; George and Coleman, 2002). Dryland salinity is also predicted to alter the behaviour of wheatbelt catchments, increasing flooding frequency and amplitude (George and Coleman, 2002).

In response to the threat to biodiversity posed by secondary salinisation the Government of Western Australia initiated a comprehensive biological survey of the wheatbelt under the Salinity Action Plan (Anonymous, 1996). The survey aimed to document the biodiversity patterns for a range of biota of the agricultural zone and select a number of Natural Diversity Recovery Catchments for focused actions to prevent/ameliorate biodiversity losses from secondary salinisation. The biodiversity patterns of wetland biota as a whole are reported by Halse et al. (2004). This paper provides more detailed information about the wetland flora and vegetation of the study area.

Very few detailed studies of wetland flora and vegetation have been undertaken within the study area. Halse et al. (1993) surveyed 106 wetlands located in an area broadly corresponding to the current study area (but including the Swan Coastal Plain) and described basic dominants and provided structural vegetation maps. A few studies have documented the flora and vegetation of wetlands on the extremities of the current survey area (Gibson et al., 2000; Keighery et al., 2002). Most studies have focused on individual wetlands, predominantly in terms of vegetation responses to changes in hydrology and salinity (Froend et al, 1987; Froend and McComb, 1991; Froend and van der Moezel, 1994). The general pattern of change of fresh or brackish plant communities to rising saline ground waters is an initial collapse of the overstorey species followed later by a death of understorey species as the water table rises to the surface. Salt tolerant species such as Halosarcia spp. then replace the original vegetation. The responses of the vegetation to hydrologic changes without salinisation can be as dramatic (Froend and van der Moezel, 1994).

Specific aims of the wetland vegetation survey were to:

- Document the flora of the wetlands of the study area.

- Provide a classification of wetland vegetation based on species composition and identify the primary environmental correlates of the biogeographic patterns.

- Evaluate whether wetland plant assemblages can be modeled in terms of edaphic, climatic and geomorphic variables.

- Provide a summary of the species assemblages most at risk from secondary salinisation.

\section{Study area}

The study area extends from the Murchison River in the north to Cape Arid in the south-east (Figure 1). Surveyed wetlands fell within an area of ca. 220 $000 \mathrm{~km}^{2}$, an area that included all of the Avon Wheatbelt and parts of the Mallee, Geraldton Sandplains, Esperance Sandplains, Jarrah Forest and Swan Coastal Plain bioregions as defined by Environment Australia (2000). With a few exceptions in the Coolgardie and Yalgoo bioregions, the eastern boundary of the study area follows the extent of agricultural clearing (Figure 1).

Within the study area, rainfall ranges from ca. 300 $\mathrm{mm}$ to $600 \mathrm{~mm}$. The only permanently filled wetlands occur in the high rainfall extreme southwest of the study area and even these may occasionally dry. All the remaining wetlands surveyed dry-out in most years, or only fill episodically. Active rivers only occur in the zones of rejuvenated drainage west of the Meckering line and along the south coast (Figure 2). Both the naturally saline south flowing streams and rivers along the south coast and most of originally fresh to brackish west flowing streams have seen varying levels of salinisation (Schofield et al., 1988). Freshwater streams persist within intact Jarrah Forest catchments. All streams cease to flow during summer, with larger streams and rivers reduced to 


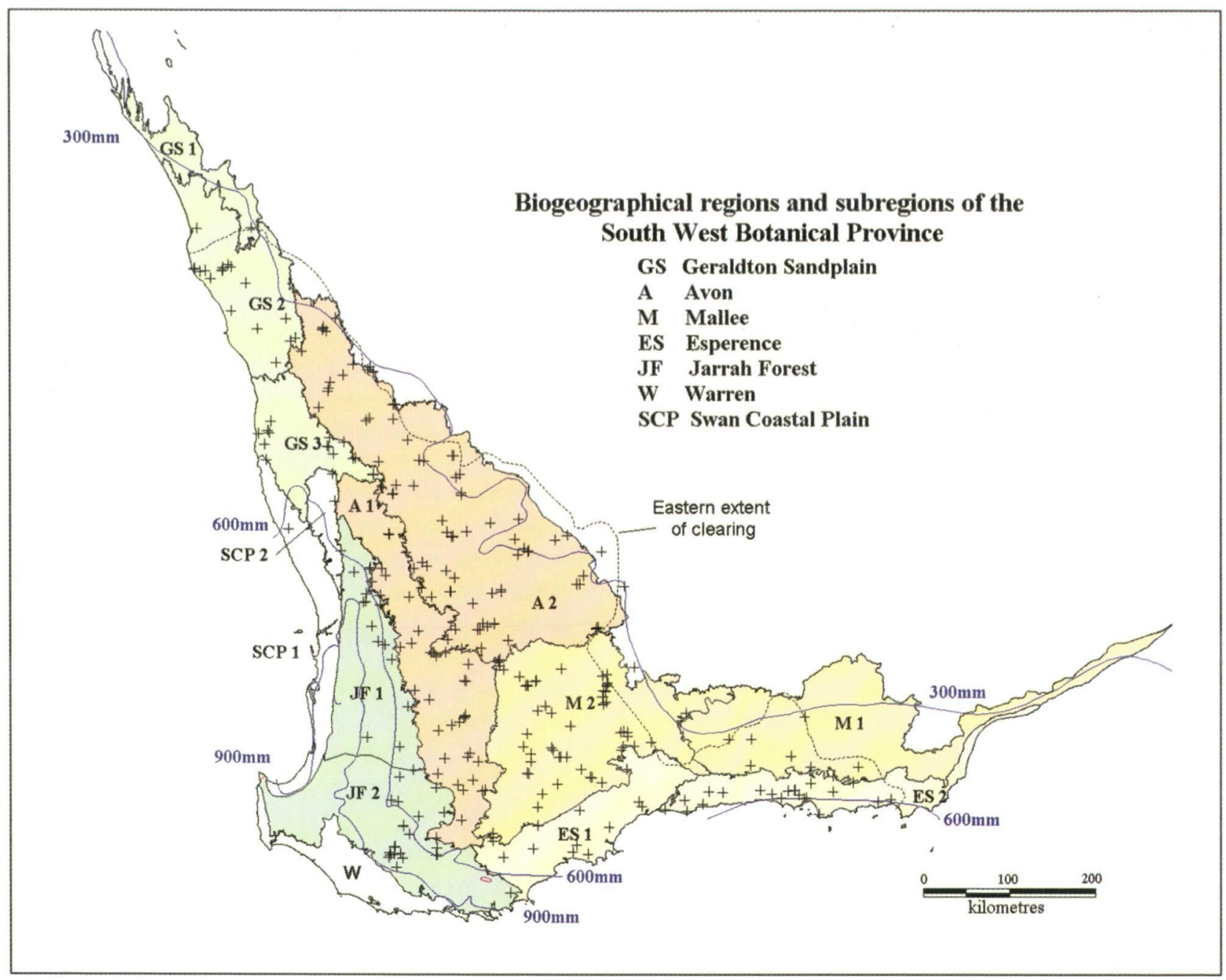

Figure 1 Map showing the distribution of quadrats within the South West Botanical Province (Beard, 1980). The bioregions and sub-regions shown (IBRA regions) are those defined by Environment Australia (2000). Seventeen quadrats occurred beyond the eastern boundary of the province, within the adjacent Yalgoo and Coolgardie bioregions. The study area broadly corresponds to the area between the $600 \mathrm{~mm}$-rainfall isohyet and the eastern extent of broadscale clearing.

a series of very isolated pools (Lane and McComb, 1988).

The inland paleo-rivers contain a diverse array of mostly naturally saline wetlands. They range from large playas with beds covering many tens of square kilometres to mosaics of small pans, many with interconnecting braided channels. These systems contain a distinctive array of aeolian and fluvial landforms that present a complex mosaic of soils, that coupled with varying elevation, drives complex vegetation patterns.

On the downwind edges of many playas and pans material transported from the lake floor forms berms and low flats of gypsum, clay and sand (Bettenay, 1962). Gypsum deposits occur within inland saline playas and coastal barred basins, where brines are concentrated and evaporites formed (Jones, 1994). At similar elevations on the windward side of lakes, low sandy flats overlay the saline clay sediments of the lakebed. These shallow beds of coarse material are also common in braided channels and are often leached and relatively nonsaline. Lunettes occur further from the lake on the downwind side and may be several metres (1-10 $\mathrm{m})$ in elevation. The composition of lunette soils reflects the prevailing hydrological regime in the wetland at the time of their formation (Bowler, 1983). Some are sandy, and show significant soil development, while others contain clays and gypsum. Sandy lunettes also occur on the margins of many higher rainfall basin wetlands (Beard, 1982). Evaporite deposits are typical features of arid and semi-arid lake dunes.

Chenopod communities dominate the fringes of these saline systems, giving way to Melaleuca and Acacia dominated shrublands and eucalypt woodlands at higher elevations. Prior to the current survey the vegetation and flora of these communities had not been systematically documented, except for taxonomic studies in the 

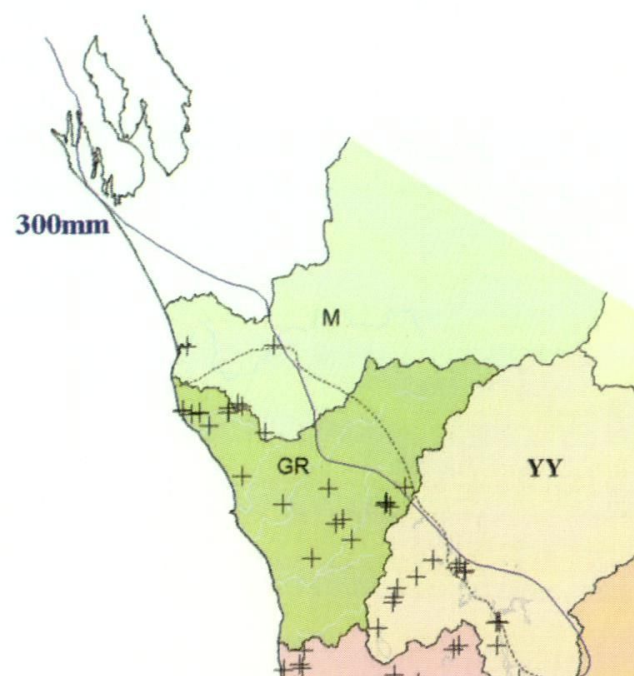

Meckering line

\section{Major catchment basins of southwestern Australia}

$\begin{array}{llll}\text { YY Yarra Yarra } & \text { MR } & \text { Murray River } \\ \text { N Ninghan } & \text { CR } & \text { Collie River } \\ \text { AV Avon River } & \text { BR } & \text { Balckwood River } \\ \text { YI Yilgarn } & \text { KR } & \text { Kent River } \\ \text { LO Lochart } & \text { WR } & \text { Warren River } \\ \text { M Murchison River } & \text { SR } & \text { Shannon River } \\ \text { GR Greenough River } & \text { FR } & \text { Frankland River } \\ \text { MHR Moore-Hill Rivers } & \text { AC } & \text { Albany Coast } \\ \text { SCP Swan coastal plain } & \text { EC } & \text { Esperence Coast } \\ & \text { SLB } & \text { Salt Lake Basin }\end{array}$

$600 \mathrm{n}$
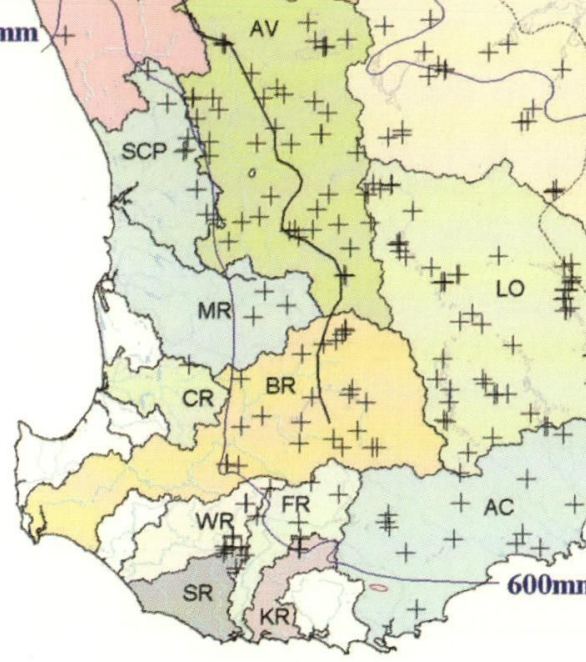

SLB

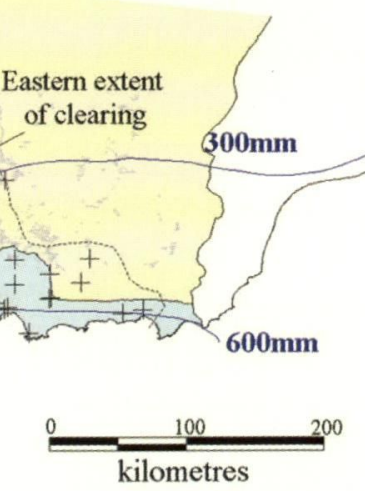

Figure 2 Map showing the distribution of quadrats within the study area, major catchment basins (Public Works Department, 1984), major salt lake systems and rivers, and the approximate position of the Meckering line (Mulchay, 1967).

Chenopodiaceae (e.g. Halosarcia, Wilson, 1980), elements of the Asteraceae, e.g. (Gnephosis (Chrysocoryne), Short, 1983) and Myrtaceae (e.g. Melaleuca, Craven et al., 2004). The aquatic dominated by trees, including Melaleuca strobophylla, Yate (Eucalyptus occidentalis), Casuarina obesa and Eucalyptus camaldulensis var. obtusa.

Shallow, seasonally filling freshwater wetlands 
verrucosa, Eragrostis australasica and Muehlenbeckia florulenta (see also Gibson ct al., 2000). Many such inland wetlands are turbid and dry to cracked clay. A large number of annuals recruit and grow in the wet and drying margins of freshwater wetlands of all types.

\section{METHODS}

\section{Field sampling and data collection}

Eight hundred and thirteen quadrats were established across the study area, to sample vegetation associated with wetland basins, margins and wetland-associated landforms such as lunettes and inter-wetland flats. Seven hundred and thirteen quadrats were located across 214 wetlands, the remaining 100 quadrats sampled wetland vegetation at individual wetlands. Wetlands were selected to sample the geographic range of wetland types within the agricultural zone and were stratified to capture variation in water quality (salinity, pH), salinity type (naturally saline, secondarily saline as a result of rising saline ground waters, or fresh), size (large basins, playas and small pans) and degree of water permanence. Between one and 10 quadrats were sampled at each of these wetlands covering the major structural vegetation zones, including the wetland basin and margins. Around large playas, quadrats were placed to sample the vegetation of the different substrates associated with evaporite and non-evaporite derived parent materials.

Quadrats were typically $10 \mathrm{~m} \times 10 \mathrm{~m}$, although rectangular $5 \mathrm{~m} \times 20 \mathrm{~m}$ quadrats were used in some narrow vegetation zones. Within quadrats all vascular plant species were recorded. Most quadrats were established between August and December 1998 to 2000 , except for 11 quadrats installed in the spring of 1997. All but 89 were sampled on two occasions. These 89 included 49 quadrats within the inundated basins of wetlands. Often aquatics, particularly Ruppia and Lepilaena, were only present later in the season during the second wetland visit and it was at this time that quadrats were established. The remaining 40 quadrats included quadrats that had been severely inundated and could not be rescored, the drying margins of wetlands that were not available on the first wetland visit when water levels were high and a few quadrats to which access could not be gained on the second visit.

A soil sample from $5-15 \mathrm{~cm}$ below the surface was collected at 30 regularly spaced points within each quadrat and bulked. For inundated quadrats, large intact samples were raised through the water column and internally sub-sampled at each point. Particle size and macronutrient analyses were performed on samples according to the methods in Appendix D of
Burbidge et al. (2000). Fifteen quadrats did not have complete soils data. They included samples for which insufficient soil was collected to complete analyses, samples for which all analyses could not be undertaken due to extremely high gypsum or sodium chloride content and quadrats from which samples were not collected because they were inaccessible on the resampling visit due to high water levels. Electrical conductivity was used as a measure of salinity although it was noted that sodium chloride was not always the dominant ion in naturally saline systems.

Latitude, longitude and elevation was recorded for each quadrat using GPS accurate to $\pm 10 \mathrm{~m}$. Climate attributes for each wetland were derived from ANUCLIM (Houlder et al., 1999), and each quadrat was assigned one of the following four elevation categories (ELCODE): 1, wetland basin / floor; 2, zone of typical inundation/wave action; 3 , elevated flat inundated in extreme events; 4 , terrestrial. These categories broadly corresponded to sub-littoral, littoral, littoral/supra-littoral and supra-littoral (terrestrial) zones. In all, four location attributes, 15 edaphic and 15 estimated climatic attributes were available for each quadrat (Table 1 , Appendices 1 and 2).

\section{Data analysis}

\section{Quadrat analysis}

Quadrats were classified according to similarities in species composition (presence/absence data) using the Czekanowski coefficient and a modification of the UPGMA clustering algorithm (Czekanowski, 1909; Sneath and Sokal, 1973). Taxa occurring at single quadrats (singletons) were removed from the dataset. Preliminary analysis also revealed a group of 53 species poor quadrats ( 1 to 3 species), clustered together and constraining the remainder of the data set. Typically, these quadrats were within saline wetland basins and contained the aquatics Ruppia spp. and/or Lepilaena spp. These quadrats were excluded from the remainder of the analysis. The final analysis data set was a matrix of 760 quadrats by 980 taxa.

The distribution of association measures was strongly skewed. Under-estimated values $(>0.99)$ were recalculated using the shortest path option in PATN (Belbin, 1995) and clustered using flexible UPGMA clustering with beta set to -0.1 (Sneath and Sokal, 1973). The methods of Dufrene and Legandre (1997) were used to identify the best indicator species for each quadrat group. Monte Carlo randomization was used to determine taxa with significant INDVAL values $(P<0.05)$

\section{Species analysis}

Species were classified using the Two-step method of Austin and Belbin (1982). The 980 taxa 
Table 1 Environmental attributes used in analyses. Climatic attributes are generated using ANUCLIM (Houlder et al., 1999).

$\begin{array}{ll}\text { Site } & \\ \text { LONG } & \text { Longitude } \\ \text { LAT } & \text { Latitude } \\ \text { ELEV } & \text { Elevation (m) } \\ \text { ELCODE } & 1 \text { - wetland basin /floor } \\ & 2 \text { - zone of typical inundation / wave action } \\ & 3 \text { - elevated flat inundated in extreme events. } \\ & 4 \text { - terrestrial }\end{array}$

\begin{tabular}{ll} 
Edaphic & \\
EC & Electrical conductivity $\left(\mathrm{mS} \mathrm{m}^{-1}\right)(1: 5)$ \\
$\mathrm{PH}$ & $\mathrm{pH}\left(\mathrm{in} \mathrm{H}_{2} \mathrm{O}\right)$ \\
$\mathrm{OC}$ & Organic Carbon $(\%)$ \\
$\mathrm{N}$ & Total Nitrogen $(\%)$ \\
$\mathrm{P}$ & Total Phosphorus $(\%)$ \\
PAV & Available Phosphorus $\left(\mathrm{HCO}_{3}\right)(\mathrm{ppm})$ \\
KAV & Available Potassium $\left(\mathrm{HCO}_{3}\right)(\mathrm{ppm})$ \\
MG & Exchangeable Magnesium $(\mathrm{me} \%)$ \\
CA & Exchangeable Calcium $(\mathrm{me} \%)$ \\
NA & Exchangeable Sodium $(\mathrm{me} \%)$ \\
K & Exchangeable Potassium $(\mathrm{me} \%)$ \\
GYP & Gypsum $(\%)$ \\
CLAY & Clay $(\%)$ \\
SILT & Silt $(\%)$ \\
SAND & Sand $(\%)$ \\
Climatic & \\
MTA & Annual mean temperature $\left({ }^{\circ} \mathrm{C}\right)$ \\
MTD & Mean temperature diurnal range $\left({ }^{\circ} \mathrm{C}\right)$ \\
ISO & Isothermality \\
TAR & Annual temperature range $\left({ }^{\circ} \mathrm{C}\right)$ \\
MXTWP & Warmest period maximum temperature $\left({ }^{\circ} \mathrm{C}\right)$ \\
MNTCP & Coldest period minimum temperature $\left({ }^{\circ} \mathrm{C}\right)$ \\
MTDQ & Driest quarter mean temperature $\left({ }^{\circ} \mathrm{C}\right)$ \\
MTWRMQ & Warmest quarter mean temperature $\left({ }^{\circ} \mathrm{C}\right)$ \\
MTCQ & Coldest quarter mean temperature $\left({ }^{\circ} \mathrm{C}\right)$ \\
PANN & Mean annual precipitation $(\mathrm{mm})$ \\
PWETP & Precipitation in the wettest period $(\mathrm{mm})$ \\
PSEAS & Precipitation seasonality $(\mathrm{mm})$ \\
PWETQ & Precipitation in the wettest quarter $(\mathrm{mm})$ \\
PWRMQ & Precipitation in the warmest quarter $(\mathrm{mm})$ \\
PCLDQ & Precipitation in the coldest quarter $(\mathrm{mm})$ \\
\hline & \\
\hline
\end{tabular}

were classified into 34 assemblages based on their patterns of co-occurrence at 760 quadrats. In an attempt to minimize artifacts and delineate ecologically coherent assemblages the classification was cut at different levels within larger classification partitions. To elucidate spatial patterning of the assemblages, the proportion of each assemblage at quadrats was plotted across the study area.

\section{Assemblage modeling}

In order to examine the patterns of species richness of each assemblage within the study area in relation to measured edaphic (including elevation around wetlands) and climatic attributes, modeling was undertaken following the approach of McKenzie et al. (2004) and Gibson et al. (2004):

(1) Each assemblage was characterized in terms of the broad distributions and known habitat preferences of its component species both within wetlands and wider terrestrial environments. Within each assemblage the number of taxa categorized as wetland specific or as also occurring in non-wetland associated terrestrial habitats was determined.

(2) The relationship between species richness and assemblage composition was examined by plotting species richness against a one-dimensional ordination of each assemblage. Ordinations were performed using the SSH module in PATN (cut level 0.2 or below minimum association measure). The resulting plots were inspected, and where they showed a unimodal relationship, species richness was regarded as a useful surrogate for assemblage composition.

(3) Generalized linear modeling (GLIM package, NAG, 1986) was used to model the relationship between the species richness of each assemblage and soil and climatic attributes of each quadrat (Table 1, Appendices 1 and 2). Since species richness values represented count data, a Poisson distribution was assumed and the logarithmic link function was used. Forward stepwise regression equations were fitted with quadrat species-richness as the dependent variable. Fifteen quadrats possessed incomplete soil attribute data and were removed from the modeling procedure. Inspection of the richness data showed that none of these quadrats represented maximum occurrences for any assemblage. Assemblage richness models included up to five terms and associated polynomials, resulting in equations with a maximum of nine terms (not including the intercept). Assemblages with low numbers of taxa, few quadrat occurrences or low maximum quadrat species-richness, were modeled without polynomial terms. Model simplification proceeded by removing nonsignificant terms based on the Wald statistic ( $P$ $>0.05$ ). Quadrats to which models were sensitive were removed and changes to the significance of model terms assessed. Models were considered to be robust when quadrat removal did not lead to changes in significance or large changes in coefficient values of model terms. Stable models could not be achieved for some assemblages despite removal of multiple influential quadrats.

(4) For each assemblage, models were evaluated in terms of whether they were consistent with the known distributions and habitat preferences of the component taxa.

\section{RESULTS}

A total of 1436 taxa, within 109 families, were 


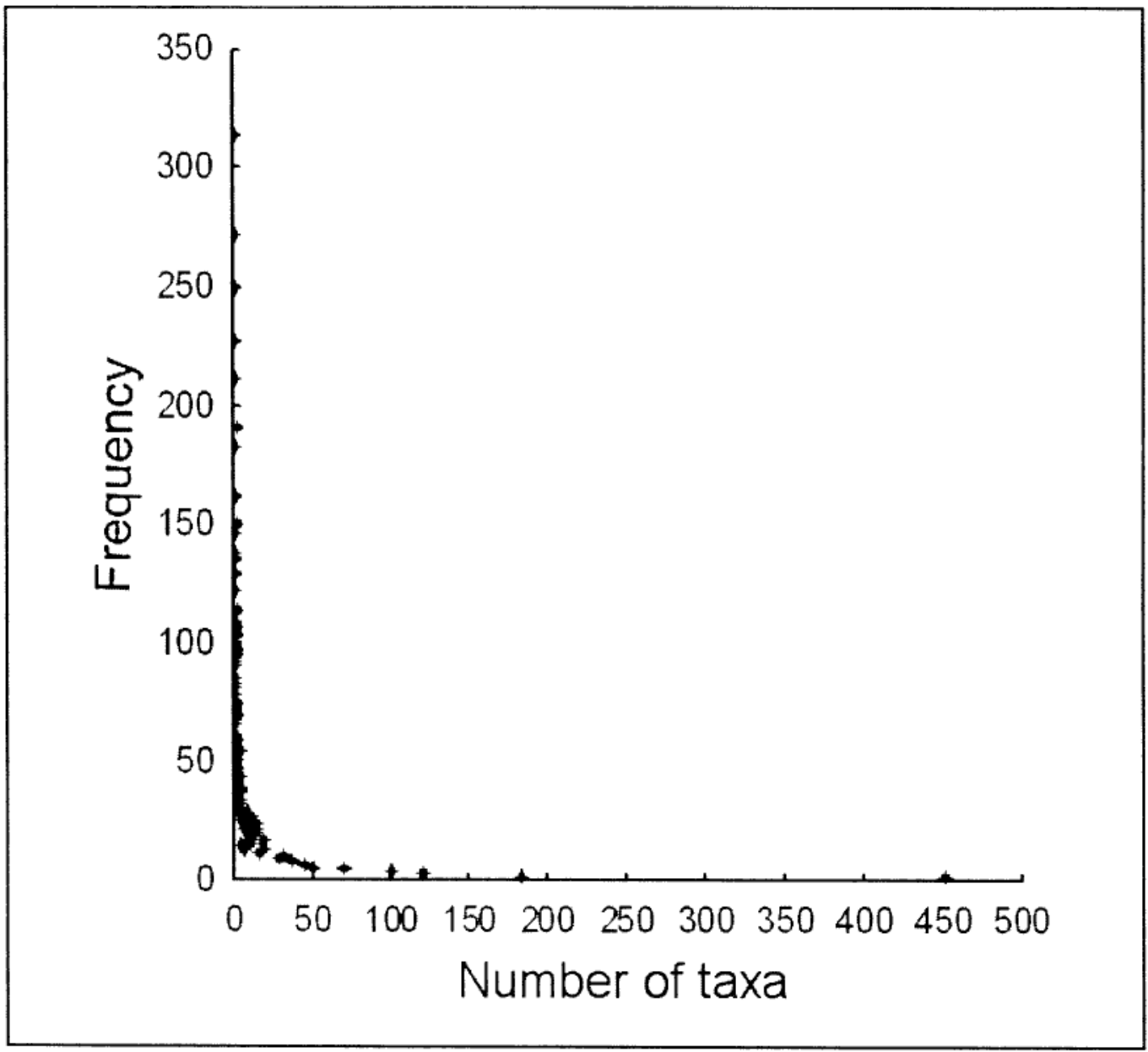

Figure 3 Frequency distribution of 1436 taxa at 813 quadrats. Approximately $30 \%$ of taxa occurred at a single quadrat (singletons) and ca. $60 \%$ of taxa occurred at less than five quadrats.

recorded from the 813 quadrats sampled (Appendix 3). Weeds (naturalized aliens) represented $11.8 \%$ of the flora.

The ten largest families were: Asteraceae (166 taxa, $11.5 \%$ of the flora), Myrtaceae $(130,9.0 \%$ ), Poaceae (101, 7.0\%), Chenopodiaceae (85, 5.9\%), Papilionacaeae $(79,5.5 \%)$, Cyperaceae $(69,4.8 \%)$, Orchidaceae $(51,3.5 \%)$, Mimosaceae $(49,3.4 \%)$, Proteaceae (34, 2.4\%) and Apiaceae (33, 2.3\%). These ten families contributed $55 \%$ of the total flora, with the remaining 99 families containing $45 \%$.

The flora comprised a total of 476 genera. The major genera included Acacia (49 taxa), Melaleuca (47), Eucalyptus (38), Stylidium (24), Halosarcia (22), Frankenia (21), Drosera (21), Triglochin (21) and Schoenus (21). The remaining 467 genera had $<20$ taxa.

The 169 weed taxa were distributed in 36 families and 111 genera. The dominant families were Poaceae (45 taxa), Asteraceae (22), Papilionacaeae (19), Caryophyllaceae (11) and Brassicaceae (8). Trifolium (10 taxa) and Bromus (6) were the major genera. Most weeds were annual herbs (87 taxa) and annual grasses (36).

Seventy-eight taxa recorded during the survey are listed on the Department of Conservation and Land Management's Priority Flora list. Of these conservation taxa, eight are gazetted as Declared Rare Flora (Appendix 3). Among them, Frankenia conferta was considered to be extinct prior to the survey. Opportunistic collecting associated with the survey also led to the rediscovery of Frankenia parvula, which had also been presumed extinct. Eight taxa were recorded for the first time during the survey. They include: Halosarcia sp. Gunyidi (M.N. Lyons 2607), Halosarcia sp. Central Wheatbelt (M.N. Lyons \& S.D. Lyons 2760), Frankenia sp. southern gypsum (M.N. Lyons 2864), Triglochin lyonsii ms (M.N. Lyons 2855), Triglochin nanum subsp. salina ms (M.N. Lyons 2833), Tribonanthes minuta ms (M.N. Lyons 2730), Hypoxis salina ms (M.N. Lyons 2735) and Thysanotus sp. Cape Le Grande (M.N. Lyons 2863). The habitats and distributions of several other taxa poorly collected prior to the current survey have been clarified. They include Halosarcia sp. Lake Moore (M.N. Lyons 2603), Calandrinia sp. Hyden (R.J.Cranfield 11298), Calandrinia sp. Needilup (K.R. Newbey 4892), Wurmbea aff. dioica "Salt Lake" (S.D.Hopper 4164), Stenopetalum salicola and Stylidium aff. obtusatum 


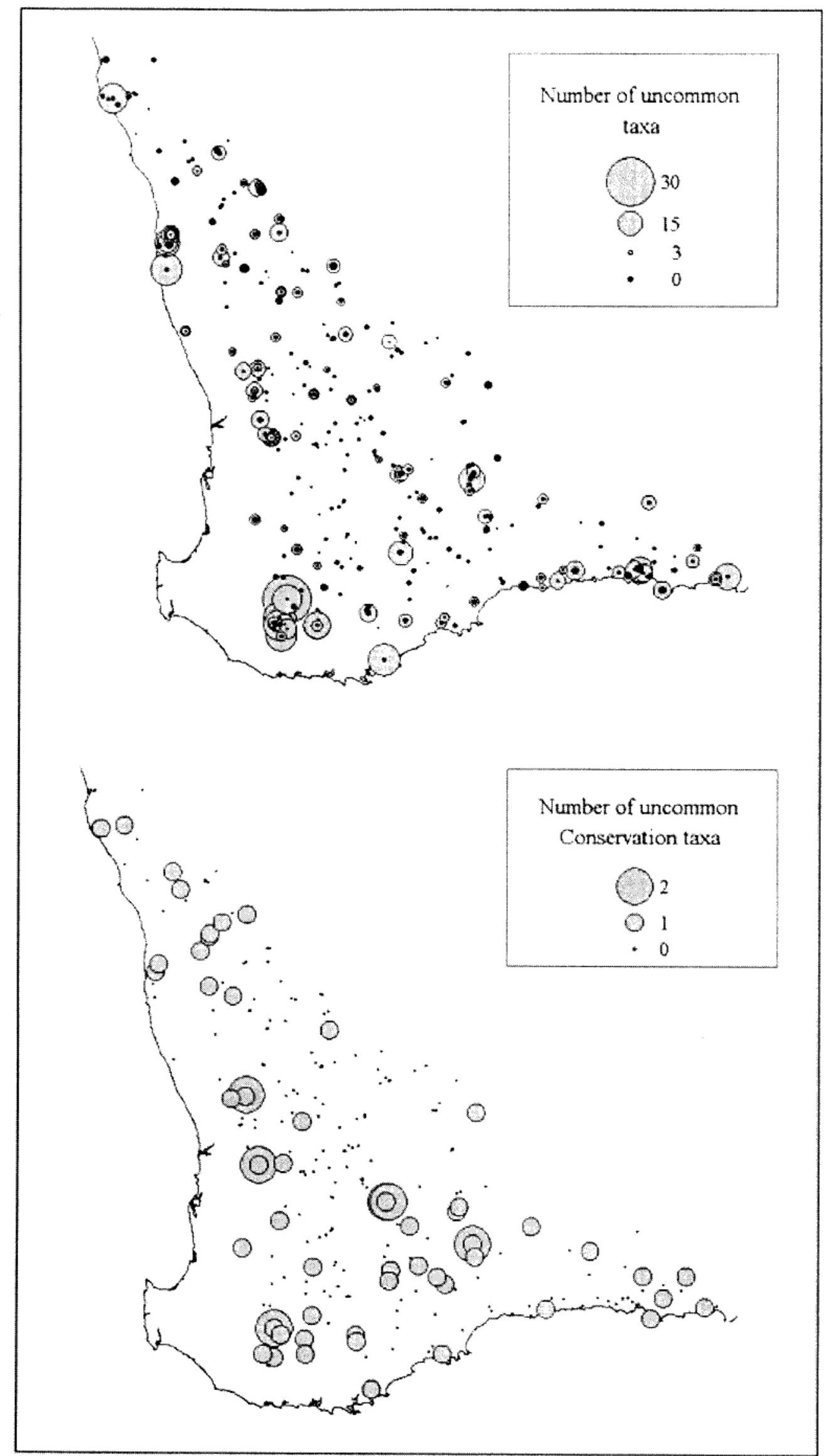

Figure 4 The geographic distribution of uncommon taxa (recorded at less than five quadrats). The upper map shows all such taxa and the lower map those listed as conservation taxa (Atkins, 2003). Conservation taxa are more evenly spread while all uncommon taxa are concentrated at the periphery of the study area. 
(M.N. Lyons 2819). The taxonomic status of several collections remain to be finalised (e.g. Genus aff. Scleria (M.N. Lyons 2926)).

\section{Species richness and frequency}

Most species occurred at very few of the 813 quadrats (Figure 3). Almost a third (31.3\%) of taxa were only recorded from a single quadrat (singletons), $59.3 \%$ at less than five quadrats and three quarters of the taxa occurred at less than ten quadrats. The most frequently recorded taxa were the weeds Hypochaeris glabra (314 quadrats), Vulpia spp. (272) and Parapholis incuroa (249). Widespread native taxa included Crassula exserta (191 quadrats), Triglochin mucronata (191) and Senecio glossanthus (183)

Five percent of quadrats contained ca. $46 \%$ of singletons and quadrats with high numbers of singletons did not occur randomly throughout the survey area. The highest numbers of singletons were concentrated in the south and west of the study area (Figure $4 a$ ). The north and eastern margin of the study area also had high numbers of low frequency taxa relative to the central core of the study area (Figure 4a). Some of the rarely recorded taxa were conservation taxa. Thirty-five of the 451 singleton taxa (7.8\%) and 54 of the 854 taxa occurring at less than five quadrats $(6.3 \%)$ were listed on CALM's Priority list. This compared to $5.4 \%$ of the total flora. Rarely recorded conservation taxa were more evenly distributed through the study area than all low frequency taxa but none the less showed a clustered distribution (Figure $4 \mathrm{~b}$ ).

Singletons might have been be predicted to occur predominately at the upland edges of wetlands, where terrestrial taxa might occasionally intrude into quadrats. This, however, was not the case. The proportion of singletons recorded between each wetland elevation was not significantly different when corrected for sampling effort (Chi-Square = 0.027 , df $=3, P>0.9$ ).

Species richness at quadrats varied dramatically from 1 to 72 taxa per quadrat with a mean of 20.5 taxa. Many quadrats in the basins of fresh and saline wetlands were extremely species poor. At a given wetland elevation, species richness varied with the electrical conductivity (EC) of the substrate (a surrogate for water column salinity at inundated quadrats). For wetland basins (ELCODE 1), species richness varied across the full range of values when EC was low but was consistently depressed at high EC (Figure 5a). A similar pattern occurred in wetland riparian zones (ELCODE 2, Figure 5b) and mid-elevation quadrats (ELCODE 3, Figure 5c). Elevated dunes and flats (ELCODE 4) showed a narrower range of $\mathrm{EC}$ and the species richness relationship was unclear (Figure $5 \mathrm{~d}$ ).

Of the 1436 taxa recorded, 1011 (70.3\%) had distributions that were not restricted to wetland habitats (Appendix 3). This group included ubiquitous taxa with broad habitat preferences such as many annuals, as well as species that

Table 2 Proportion of different life forms at four wetland elevations. Elevation 1, wetland basin /floor; 2, zone of typical inundation / wave action; 3 , elevated flat inundated in extreme events; 4 , terrestrial.

\begin{tabular}{|c|c|c|c|c|c|}
\hline Lifeform & $\begin{array}{c}\text { Elevation } 1 \\
(\%)\end{array}$ & $\begin{array}{c}\text { Elevation } 2 \\
(\%)\end{array}$ & $\begin{array}{c}\text { Elevation } 3 \\
(\%)\end{array}$ & $\begin{array}{c}\text { Elevation } 4 \\
(\%)\end{array}$ & $\begin{array}{c}\text { All elevations } \\
(\%)\end{array}$ \\
\hline cycad & - & 0.1 & 0.1 & - & 0.1 \\
\hline arboresent monocot & - & 0.1 & 0.2 & 0.1 & 0.1 \\
\hline annual grass & 4.7 & 4.2 & 4.3 & 4.2 & 3.4 \\
\hline annual herb & 39.6 & 33.9 & 33.3 & 30.7 & 29.3 \\
\hline annual sedge & 6.5 & 2.3 & 2.2 & 0.8 & 2.2 \\
\hline parasitic annual herb & 0.5 & 0.7 & 0.5 & 0.4 & 0.4 \\
\hline ferns & 1.6 & 0.4 & 0.6 & 0.5 & 0.6 \\
\hline fern allies & 0.8 & 0.3 & 0.1 & - & 0.4 \\
\hline geophyte & 8.0 & 8.7 & 7.6 & 7.9 & 8.1 \\
\hline parasitic perennial herb & - & 0.1 & 0.2 & 0.1 & 0.1 \\
\hline perennial climber & 0.3 & 0.2 & 0.6 & 0.4 & 0.4 \\
\hline perennial grass & 1.8 & 3.4 & 3.8 & 4.1 & 3.5 \\
\hline perennial herb & 9.1 & 9.7 & 10.6 & 8.0 & 10.4 \\
\hline perennial restiad & 2.6 & 2.0 & 1.2 & 1.2 & 1.9 \\
\hline perennial sedge & 7.0 & 4.1 & 2.4 & 1.9 & 3.4 \\
\hline shrub & 14.2 & 26.3 & 28.2 & 34.4 & 31.5 \\
\hline shrub / small tree & 0.8 & 0.6 & 0.7 & 1.0 & 0.6 \\
\hline mallee & 0.3 & 0.9 & 1.3 & 2.0 & 1.4 \\
\hline tree & 1.8 & 1.8 & 1.4 & 1.8 & 1.6 \\
\hline \multirow[t]{2}{*}{ climbing shrub } & 0.5 & 0.3 & 0.7 & 0.4 & 0.6 \\
\hline & 100 & 100 & 100 & 100 & 100 \\
\hline
\end{tabular}




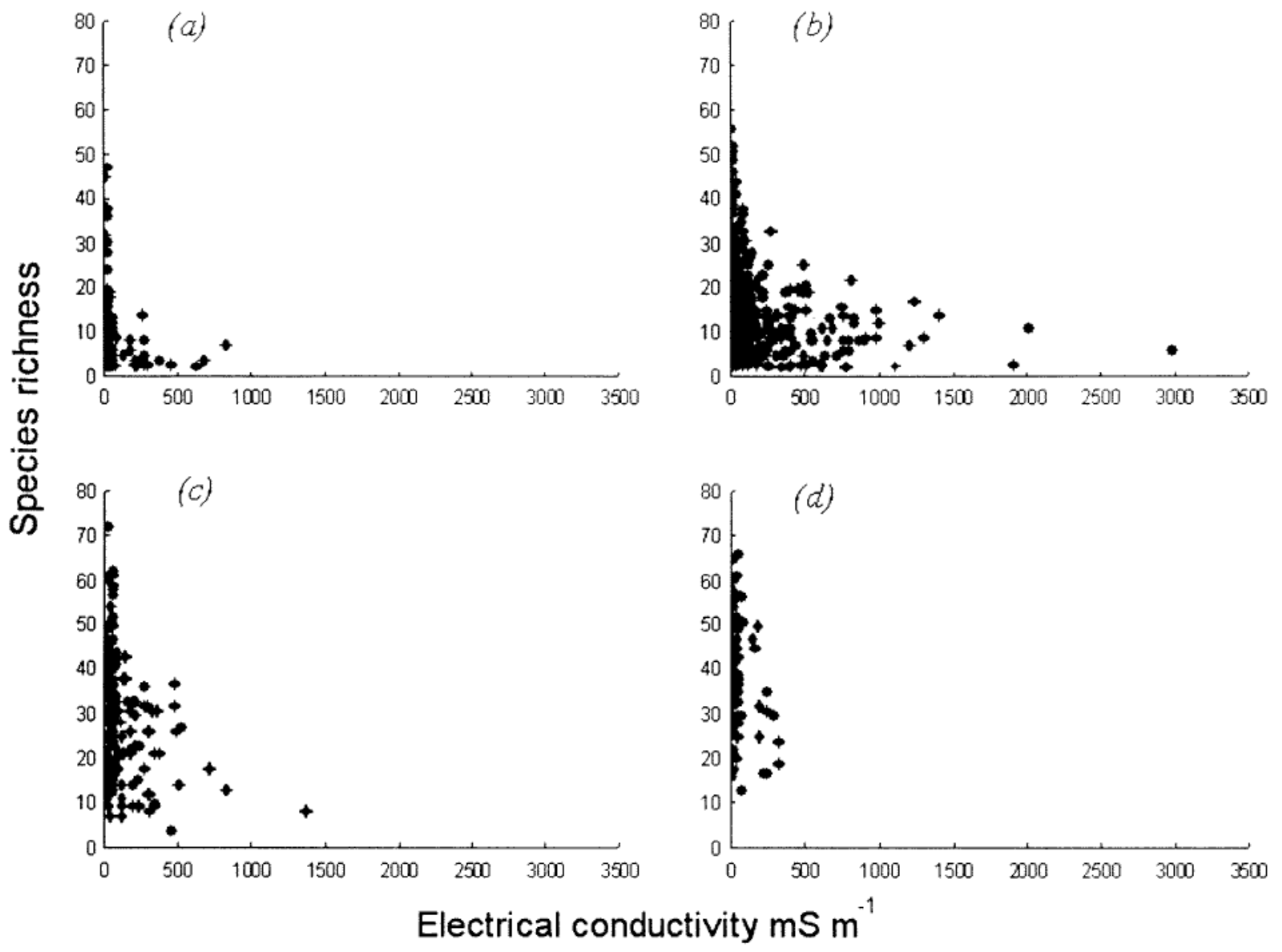

Figure 5 Quadrat species richness plotted against substrate electrical conductivity (EC) at each wetland elevation. (a) wetland basins - sub-littoral, (b) zone of inundation - littoral, (c) elevated flat - littoral/supra littoral and (d) terrestrial - supra littoral. Species richness is constrained at high substrate EC particularly at low elevations.

largely occur around wetlands but have distributions that extend to other mesic habitats such as valley floors (particularly in the higher rainfall south-west) and granite rock margins. The remaining 428 taxa $(29.7 \%)$ were restricted to wetlands (Appendix 3). These obligate wetland taxa included aquatic and amphibious plants (see below), in combination with species that only occur in riparian zones, or on wetland-related geomorphologic features such as lunettes and gypsum deposits.

Aquatic and amphibious plants represented a small proportion $(9.9 \%)$ of the total flora recorded (Appendix 3). Aquatics were defined as taxa that do not tolerate drying. They could be floating (e.g. Lemna sp.), submerged (Potamogeton, Ruppia, Lepilaena spp.) or emergent (Triglochin, Villarsia). Taxa that tolerated both drying and flooding were defined as amphibious. Most amphibious taxa were shrubs (e.g. Melaleuca spp., Muehlenbeckia spp., Halosarcia spp. and Tecticornia spp.), perennial sedges (e.g. Baumea spp.) or rushes (Juncus spp.). The large number of annual herbs that recruit in wet terrestrial habitats but do not persist when inundated were not included in the latter category.

Water quality data were not collected for all wetlands and riparian zones dominated by amphibious taxa were not always inundated. Waterbody and substrate EC were both available at 31 quadrats where aquatics and/or amphibious taxa were recorded. The two parameters were highly correlated $(\mathrm{r}=0.88, \mathrm{n}=31, P<0.00001)$. Few aquatics and amphibious taxa were present at high substrate EC (Figure 6). At low EC richness varied over the full range (1-11 taxa). The richest quadrats for aquatic and amphibious taxa were fresh wetlands in the high rainfall south-west of the study area. Species of Ruppia and Lepilaena dominated the aquatic flora of saline wetlands.

Across all quadrats, shrubs (31.5\%), annual herbs (29.3\%) and perennial herbs (10.4\%) were the most abundant life forms of the 1439 taxa recorded. Annual herbs (39.6\%) and perennial sedges (7.0\%) were more abundant at lower wetland elevations. Shrubs $(34.4 \%)$ were more abundant at high elevations (Table 2). 

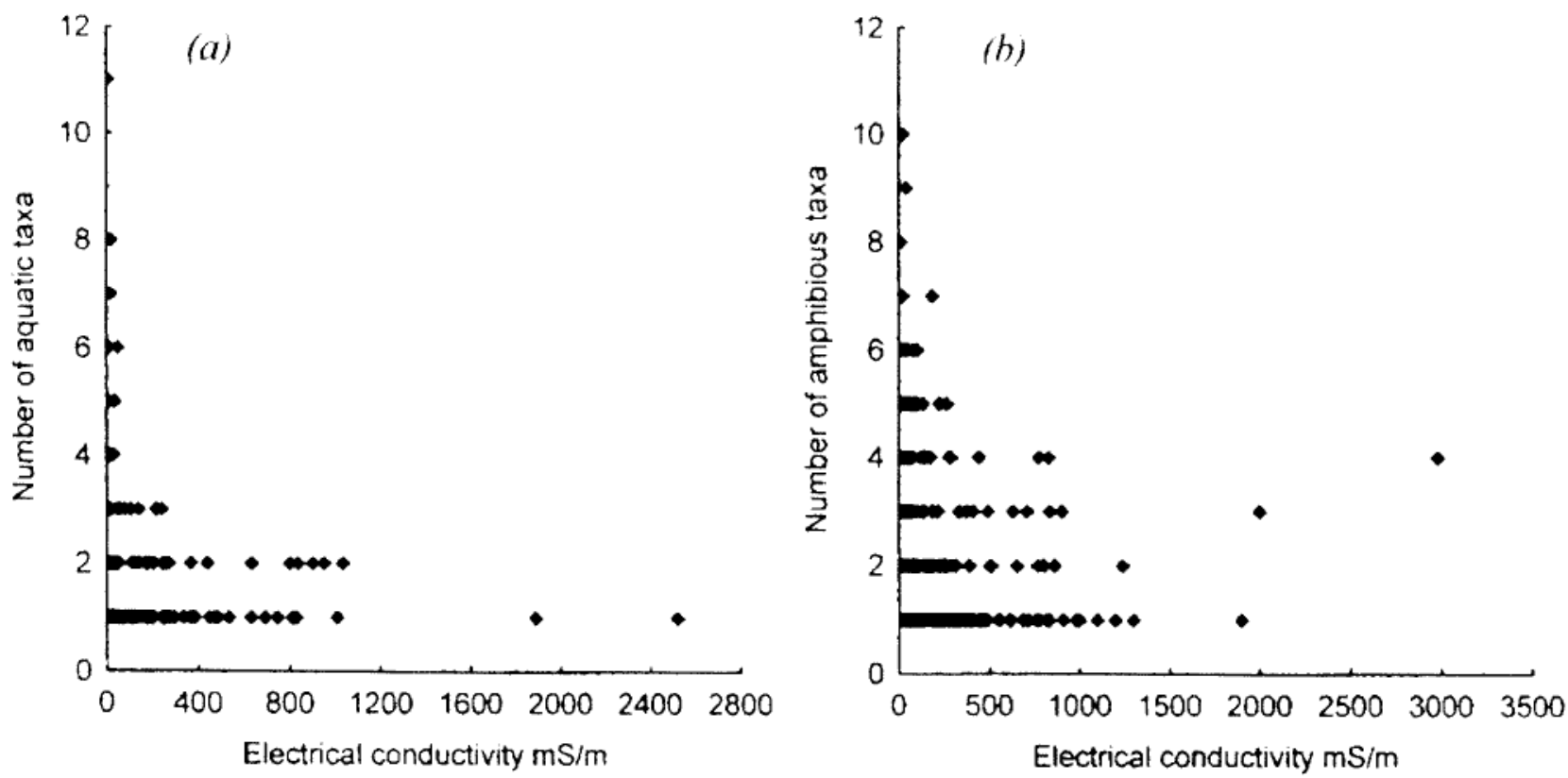

Figure 6 The number of (a) aquatic and (b) amphibious taxa per quadrat plotted against substrate electrical conductivity for 813 quadrats. Species richness is constrained as electrical conductivity increases for both lifeforms.

\section{Quadrat classification}

The quadrat classification for the reduced (760 quadrats $\times 980$ taxa) dataset was examined at three levels. The 12 and 26 group level classification is shown in Figure 7. At the 26-group level additional structure was discernable within some larger quadrat groups (groups 1, 8, 9, 20, 22 and 25). These were subdivided to produce a final 39-group classification. The 39-group classification is detailed below. Major headings correspond to the 12 group level classification (see Figure 7). Taxa with high INDVAL scores for each group were described as typical of each quadrat group.

Woodlands and shrublands of lunettes, inter-zetlands flats and rises - predominantly inland naturally saline systems

A large group of 195 quadrats occupying dunes, elevated flats and some riparian zones of mostly primaryily saline and some secondarily saline wetlands formed the first partition of the 12-group classification. They largely occurred in the Avon Wheatbelt and Mallee Bioregions (Table 3, Figure 7). Quadrats included eucalypt woodlands, Melaleuca, Acacia, Hakea and Eremophila tall shrublands and chenopod shrublands dominated by Maireana, Sclerolaena and Halosarcia

Group 1.1 Central Melaleuca shrublands on wetland margins. Group 1.1 was a heterogeneous group of quadrats, occurring on the margins of primary salt lakes and saline playas. The group occurs in the central part of the study area, within the Avon Wheatbelt, southern Geraldton Sandplains 3 and Mallee 2 bioregions (Table 3). The group is distinguished from other Melaleuca shrublands by the lack of many upland herbs (cf. group 1.3) and the presence of species typical of lower elevations. Species with high INDVAL scores include Triglochin elongatum $\mathrm{ms}$, Rhagodia drummondii and Gunniopsis intermedia. Elements of lower elevations recorded at these quadrats included Cotula cotuloides, C. bipinnata, Parapholis incurva and Halosarcia pergranulata (Appendix 4). Some quadrats in the group had been inundated during flood events, while other showed signs of secondary salinisation.

Group 1.2 Woodlands and shrublands on southern lunettes and rises. Group 1.2 was comprised of quadrats centred within the Mallee 2 bioregion, occurring on elevated flats and dunes predominantly in naturally saline lake systems. Quadrats were relatively species rich (36.5 species/ quadrat). Typical species (high INDVAL scores) include Bossiaea halophila, Helichrysum leucopsideum, Ptilotus sp. Salt Lake (M. Graham G200.28) and Podotheca angustifolium. Canopy species included Melaleuca thyoides, Melaleuca brophyi, Eucalyptus spathulata and $E$. sargentii.

Group 1.3 Shrublands on northern and central lunettes and rises. Group 1.3 was comprised of a suite of shrublands that variably contained the structural dominants Acacia eremaea, Eremophila miniata, E. oldfieldii and Melaleuca atroviridis $\mathrm{ms}$. Quadrats occurred on elevated dunes, rises and elevated flats between wetlands. This group occurred almost exclusively at wetlands within inland paleo-drainage systems. The group mainly occurs within the Avon 2 bioregion and showed the 


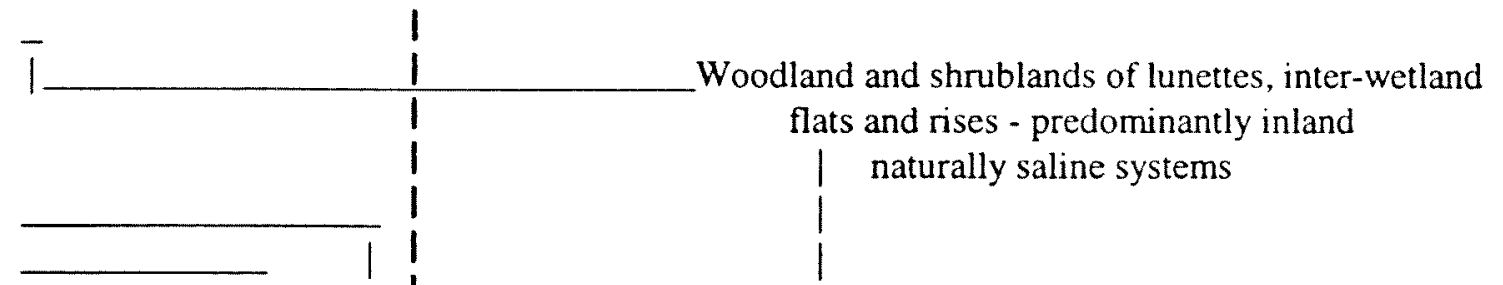

3 (11)

$4(3)$

$5(7)$

$6(4)$

$7(10)$

8 (93)

$9(121)$

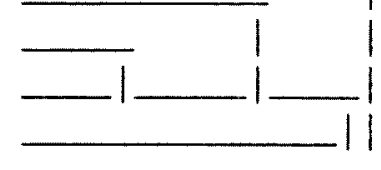

Species poor, chenopod dominated berms, beaches, and gypsum flats of natural and degraded

\author{
saline wetlands
}<smiles>I[IH]</smiles>

Species rich strands, berms, and low sandy rises of naturally saline and degraded

10 (17)

$11(2)$

$12(11)$

$13(11)$

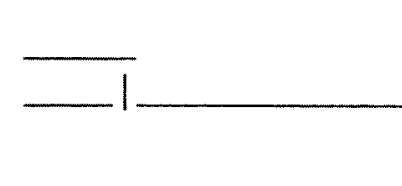

$14(43)$

15 (9)

$16(5)$

17 (38)

$18(3)$

19 (21)

20 (27)

21 (17) 
Table 3 Occurrence of quadrats at the 39 group level classification, within IBRA sub-regions (Environment Australia, 2000; see also Figure 1). A, Avon Wheatbelt; M, Mallee; GS, Geraldton Sandplains; SCP, Swan Coastal Plain; JF, Jarrah Forest; ES, Esperance Sandplain; C, Coolgardie; Y, Yalgoo.

\begin{tabular}{|c|c|c|c|c|c|c|c|c|c|c|c|c|c|c|c|c|}
\hline Group & A1 & $\mathrm{A} 2$ & M1 & M2 & GS1 & GS2 & GS3 & SCP1 & $\mathrm{SCP} 2$ & JF1 & JF2 & ES1 & ES2 & C & $\mathbf{Y}$ & $\mathrm{n}$ \\
\hline 1.1 & 1 & 15 & - & 5 & - & - & 5 & - & - & - & - & - & - & - & - & 26 \\
\hline 1.2 & - & 1 & 3 & 14 & - & - & - & - & - & - & - & 1 & - & 1 & - & 20 \\
\hline 1.3 & - & 33 & - & 4 & - & 1 & 1 & - & - & - & - & - & - & - & - & 39 \\
\hline 1.4 & 7 & 1 & - & 1 & - & - & 2 & - & - & 1 & - & - & - & - & - & 12 \\
\hline 1.5 & - & 2 & - & - & - & - & - & - & - & - & - & - & - & 2 & - & 4 \\
\hline 1.6 & - & 4 & - & 2 & - & - & 1 & - & - & - & - & - & - & - & - & 7 \\
\hline 1.7 & - & 27 & - & - & - & - & - & - & - & - & - & - & - & 2 & 1 & 30 \\
\hline 1.8 & 1 & 15 & - & 22 & - & - & - & - & - & - & - & $\ldots$ & - & 1 & - & 39 \\
\hline 1.9 & - & - & - & 3 & - & - & - & - & - & - & - & 3 & - & - & - & 6 \\
\hline 2 & - & 2 & - & 2 & - & 1 & 5 & - & 2 & - & - & - & - & - & - & 12 \\
\hline 3 & - & 9 & - & 1 & - & - & - & - & - & - & - & - & - & - & 1 & 11 \\
\hline 4 & - & - & 2 & - & - & - & - & - & - & - & - & - & 1 & - & - & 3 \\
\hline 5 & - & 6 & - & 1 & - & - & -.. & - & - & - & - & - & - & - & - & 7 \\
\hline 6 & - & - & 2 & 2 & - & - & - & - & - & - & - & - & - & - & - & 4 \\
\hline 7 & - & 6 & 2 & 2 & - & - & - & - & - & - & - & - & - & - & - & 10 \\
\hline 8.1 & 1 & 38 & - & 9 & - & 5 & 7 & - & - & - & - & - & - & - & - & 60 \\
\hline 8.2 & - & 22 & - & 8 & - & - & - & - & - & - & - & - & - & 2 & 1 & 33 \\
\hline 9.1 & 40 & 17 & - & 23 & - & - & 3 & - & 2 & 5 & - & 2 & 1 & - & - & 93 \\
\hline 9.2 & 1 & 1 & 3 & 14 & - & - & 5 & - & - & - & - & 2 & 2 & - & - & 28 \\
\hline 10 & 7 & 1 & - & 2 & - & 1 & - & - & 2 & - & - & 2 & 2 & - & - & 17 \\
\hline 11 & - & 1 & - & 1 & - & - & - & - & - & - & - & - & - & - & - & 2 \\
\hline 12 & 1 & 5 & 1 & 2 & - & 1 & - & - & - & - & 1 & - & - & - & - & 11 \\
\hline 13 & - & 4 & - & 2 & - & - & 2 & - & - & - & 1 & - & 2 & - & - & 11 \\
\hline 14 & 17 & 8 & - & - & - & 4 & 1 & - & - & 4 & 4 & 2 & 3 & - & - & 43 \\
\hline 15 & 5 & 4 & - & - & - & - & - & - & - & - & - & - & - & - & - & 9 \\
\hline 16 & - & - & - & - & 2 & - & - & - & - & - & - & - & - & - & 3 & 5 \\
\hline 17 & 1 & 9 & 1 & 12 & - & 7 & 6 & - & - & - & - & - & - & 2 & - & 38 \\
\hline 18 & - & - & - & 2 & - & 1 & - & - & - & - & - & - & - & - & - & 3 \\
\hline 19 & - & - & - & - & - & - & 1 & - & 1 & 11 & 8 & - & - & - & - & 21 \\
\hline 20.1 & - & 1 & - & - & - & - & - & 2 & - & 4 & 9 & - & - & - & - & 16 \\
\hline 20.2 & - & - & - & - & - & - & 2 & - & - & 5 & 3 & 1 & - & - & - & 11 \\
\hline 21 & 3 & - & - & - & - & - & - & - & - & 2 & 9 & - & 3 & - & - & 17 \\
\hline 22.1 & - & - & - & - & - & 4 & 1 & - & - & - & - & 2 & 1 & - & - & 8 \\
\hline 22.2 & - & 3 & - & - & - & 9 & 1 & - & - & - & - & - & 1 & - & - & 14 \\
\hline 23 & - & - & - & - & - & - & - & - & - & - & 1 & - & 2 & - & - & 3 \\
\hline 24 & - & - & - & - & - & 4 & - & - & - & - & - & - & - & - & - & 4 \\
\hline 25.1 & - & - & - & 1 & - & - & - & - & - & - & 4 & 4 & 12 & - & - & 21 \\
\hline 25.2 & - & - & - & 1 & - & - & - & - & - & - & 2 & 14 & 31 & - & - & 48 \\
\hline 26 & - & 3 & - & 6 & - & - & 3 & - & - & - & $\overline{1}$ & - & - & 1 & - & 14 \\
\hline
\end{tabular}

highest mean species richness of all groups $(48.5$ taxa/quadrat). Soil $\mathrm{pH}$ and $\mathrm{EC}$ are relatively low and gypsum was not detected in any quadrat. Based on INDVAL scores, herbs dominate the species typical of the quadrat group and include Nicotiana occidentalis, Velleia cycnopotamica and Ptilotus humilis Typical shrubs include Gunniopsis quadrifida, Scaevola spinescens and Rhagodia drummondii.

Group 1.4 Woodlands and shrublands on western lunettes and rises. A group of mostly woodland quadrats on dunes and rises with very similar soil properties to group 1.3 (Table 4). A suite of herbs including Stypandra glauca, Opercularia vaginata and Parietaria cardiostegia defines the group. It is centred in the Avon 1 and adjacent margins of the Jarrah Forest, Geraldton Sandplains and Mallee bioregions. Typical species (high INDVAL scores) are also structural dominants and include Eucalyptus loxophleba, Acacia acuminata and Allocasuarina huegeliana. The quadrat group is the higher rainfall analogue of groups 1.2 and 1.3.

Group 1.5 Jacksonia arida ms shrublands. This group was a small group of four quadrats in the extreme northeast of the study area on the margins of saline playas. The presence of Aristida contorta, Podotheca gnaphalioides, Goodenia berardiana and Jacksonia arida ms links the quadrats.

Group 1.6 Darwinia halophila shrublands. Group 1.6 was a small heterogeneous group of quadrats on dunes of inland saline pans and playas. Alyxia buxifolia and Darwinia halophila show high INDVAL scores for this group (Appendix 4). The group includes two quadrats with very high $E C$ and gypsum content. 
Table 4 Mean and SE values of environmental attributes for 39 quadrat groups (see Table 1 for explanation of parameters): Where means are based on a reduced number of quadrats, values are annotated as $N^{*}$ and $N^{* *}$ (see bottom of table for values of $\mathrm{N}$ ). Vertical lines denote division at the twelve group level.

\begin{tabular}{|c|c|c|c|c|c|c|c|c|c|c|c|c|c|c|}
\hline Group & 1.1 & 1.2 & 1.3 & 1.4 & 1.5 & 1.6 & 1.7 & 1.8 & 1.9 & 2 & 3 & 4 & 5 & 6 \\
\hline \multirow[t]{2}{*}{ SPPRICH } & 34.62 & 36.45 & 48.46 & 38.50 & 24.25 & 29.14 & 36.23 & 28.05 & 18.67 & 18.58 & 16.73 & 7.67 & 7.00 & 13.00 \\
\hline & 11.17 & 9.71 & 7.99 & 8.55 & 763 & 10.64 & 10.32 & 8.45 & 8.59 & 8.81 & 8.36 & 0.58 & 3.83 & 1.15 \\
\hline \multirow[t]{2}{*}{ EC } & 53.54 & 42.11 & 31.11 & 24.78 & 19.85 & 107.56 & 68.77 & 138.80 & 92.32 & 18.08 & 277.54 & 517.33 & 183.31 & 262.75 \\
\hline & 64.17 & 48.80 & 19.74 & 55.81 & 25.60 & 128.80 & 74.64 & 137.85 & 114.65 & 17.94 & 130.23 & 288.82 & 115.41 & 56.87 \\
\hline \multirow[t]{2}{*}{$\mathrm{pH}$} & 6.41 & 6.50 & 6.23 & 6.59 & 6.30 & 5.53 & 6.94 & 7.22 & 7.60 & 6.74 & 8.42 & 8.67 & 5.21 & 6.55 \\
\hline & 0.64 & 0.71 & 0.62 & 0.88 & 0.35 & 1.39 & 0.85 & 1.10 & 0.83 & 0.36 & 0.33 & 0.58 & 0.59 & 0.78 \\
\hline \multirow[t]{2}{*}{ OC } & 0.85 & 0.90 & 0.76 & 0.88 & 0.73 & 0.60 & 0.48 & 1.03 & 2.15 & 0.54 & 0.47 & 1.50 & 0.60 & 0.40 \\
\hline & 0.51 & 0.31 & 0.36 & 0.43 & 0.48 & 0.21 & 0.22 & 0.65 & 1.01 & 0.34 & 0.46 & 1.32 & 0.27 & 0.12 \\
\hline \multirow[t]{2}{*}{ N } & 0.04 & 0.04 & 0.04 & 0.06 & 0.03 & 0.04 & 0.03 & 0.07 & 0.12 & 0.03 & 0.05 & 0.13 & 0.02 & 0.02 \\
\hline & 0.02 & 0.02 & 0.02 & 0.02 & 0.02 & 0.02 & 0.01 & 0.05 & 0.05 & 0.01 & 0.05 & 0.14 & 0.01 & 0.01 \\
\hline $\mathrm{P}$ & 51.54 & 45.85 & 60.36 & 53.08 & 43.25 & 58.00 & 73.30 & 107.10 & 87.67 & 41.50 & 129.00 & 105.67 & 47.71 & 47.50 \\
\hline & 45.28 & 46.44 & 20.04 & 22.28 & 23.00 & 19.91 & 25.16 & 58.58 & 35.22 & 31.42 & 79.78 & 99.57 & 18.73 & 41.40 \\
\hline PAV & 1.69 & 1.53 & 2.23 & 2.13 & 1.10 & 1.51 & 3.27 & 6.93 & 4.53 & 2.85 & 20.82 & 4.33 & 1.91 & 1.35 \\
\hline & 1.41 & 1.27 & 1.08 & 1.67 & 1.04 & 1.42 & 1.35 & 6.43 & 3.40 & 4.30 & 14.82 & 2.31 & 1.60 & 1.39 \\
\hline $\mathrm{KAV}$ & 104.42 & 102.95 & 127.90 & 101.42 & 67.00 & 136.86 & 213.37 & 465.67 & 1105.00 & 93.33 & 309.18 & 490.00 & 164.29 & 124.00 \\
\hline & 104.51 & 100.67 & 73.22 & 80.27 & 47.66 & 59.49 & 138.46 & 427.88 & 1159.20 & 108.46 & 189.59 & 182.48 & 36.45 & 100.77 \\
\hline $\mathrm{Mg}$ & 1.32 & 1.10 & 1.03 & 0.90 & 0.66 & 0.93 & 1.28 & 2.82 & 6.01 & 0.67 & 2.12 & 5.10 & 0.98 & 0.72 \\
\hline & 1.29 & 0.71 & 0.46 & 0.72 & 0.36 & 1.41 & 0.73 & 1.88 & 3.20 & 0.46 & 1.60 & 1.33 & 0.47 & 0.46 \\
\hline $\mathrm{Ca}$ & 1.33 & 2.92 & 1.63 & 3.22 & 1.99 & 3.73 & 1.08 & 5.26 & 8.21 & 1.47 & 8.82 & 3.24 & 2.42 & 10.83 \\
\hline & 1.45 & 3.52 & 1.15 & 2.14 & 1.60 & 3.94 & 1.24 & 5.59 & 4.25 & 1.17 & 4.57 & 1.84 & 3.38 & 5.69 \\
\hline $\mathrm{Na}$ & 0.81 & 0.62 & 0.54 & 0.31 & 0.29 & 0.60 & 0.83 & 1.88 & 3.88 & 0.54 & 1.5 & 7.3 & 0.83 & 0.74 \\
\hline & 0.54 & 0.46 & 0.29 & 0.26 & 0.16 & 0.39 & 0.49 & 1.62 & 4.80 & 0.36 & 1.64 & 7.40 & 0.35 & 0.45 \\
\hline $\mathrm{K}$ & 0.21 & 0.20 & 0.27 & 0.24 & 0.14 & 0.28 & 0.58 & 1.27 & 3.76 & 0.2 & 0.6 & 1.29 & 0.30 & 0.20 \\
\hline & 0.17 & 0.22 & 0.17 & 0.24 & 0.08 & 0.16 & 0.55 & 1.40 & 4.63 & 0.30 & 0.63 & 0.64 & 0.11 & 0.20 \\
\hline GYP & 0.31 & 0.75 & 0.00 & 0.01 & 0.00 & 21.86 & 0.01 & 3.59 & 0.0 & 0.0 & 53.64 & 0.7 & 6.1 & 54.25 \\
\hline & 1.57 & 3.13 & 0.00 & 0.03 & 0.00 & 37.33 & 0.03 & 13.68 & 0.0 & 0.00 & 47.85 & 1.1 & 10.52 & 42.76 \\
\hline CLAY & 4.79 & $2.19^{*}$ & 3.12 & 2.96 & 2.08 & $4.74^{*}$ & 3.31 & $5.68^{*}$ & 12.41 & 2.1 & 6.08 & 3.2 & 4.7 & $2.10^{*}$ \\
\hline & 7.31 & 1.37 & 1.39 & 1.87 & 0.39 & 2.05 & 2.77 & 11.15 & 22.93 & 1.09 & 4.45 & 0.9 & 3.9 & 1.98 \\
\hline SILT & 2.18 & $1.70^{*}$ & 1.82 & 1.75 & 1.0 & $1.66^{*}$ & 2.6 & $3.67^{*}$ & 5.04 & 1.0 & 6.0 & $3.47^{*}$ & 1.30 & $1.40^{*}$ \\
\hline & 1.80 & 1.06 & 0.94 & 1.22 & 0.6 & 0.6 & 1.25 & 2.12 & 2.39 & 0.75 & 7.59 & 1.23 & 0.35 & 0.57 \\
\hline SAND & 93.03 & $96.09^{*}$ & 95.05 & 95.28 & 96.85 & $93.6^{*}$ & 94.07 & $90.65^{*}$ & 82.53 & 96.83 & 87.90 & $93.30^{*}$ & 93.99 & $96.50^{*}$ \\
\hline & 8.56 & 1.91 & 1.63 & 2.74 & 1.03 & 2.53 & 3.20 & 11.49 & 22.44 & 1.50 & 11.68 & 0.36 & 3.70 & 2.55 \\
\hline LONG & 117.75 & 119.04 & 116.67 & 117.06 & 119.13 & 117.20 & 117.18 & 118.57 & 119.05 & 116.63 & 117.22 & 122.17 & 117.97 & 120.38 \\
\hline & 1.02 & 1.20 & 0.96 & 0.73 & 0.25 & 1.16 & 0.94 & 0.83 & 0.54 & 1.90 & 0.84 & 0.39 & 1.21 & 1.02 \\
\hline LAT & -31.39 & -33.07 & -30.11 & -32.00 & -31.05 & -30.84 & -30.34 & -32.23 & -33.73 & -30.45 & -30.1 & -33 & -31.46 & -33.02 \\
\hline & 1.00 & 0.76 & 1.19 & 1.44 & 0.10 & 1.39 & 0.83 & 1.01 & 0.50 & 1.23 & 1.23 & 0.44 & 0.96 & 0.25 \\
\hline ELEV & 282.7 & 265.7 & 281.1 & 251.8 & 334.5 & 278.7 & 289.6 & 302.2 & 241.3 & 202 & 290.5 & 138.3 & 269.1 & 265.8 \\
\hline & 38.5 & 53.1 & 20.7 & 26.8 & 4.0 & 14.6 & 28.6 & 35.3 & 94.8 & 107.4 & 26.0 & 110.1 & 20.9 & 75.9 \\
\hline MTA & 17.5 & 16.0 & 18.6 & 16.9 & 17.9 & 18.0 & 18.5 & 16.8 & 15.5 & 18.4 & 18.8 & 16.0 & 17.6 & 16.0 \\
\hline & 0.9 & 0.7 & 1.0 & 1.4 & 0.1 & 1.1 & 0.7 & 1.0 & 0.2 & 1.2 & 1.1 & 0. & 0.8 & 0.2 \\
\hline MTD & 13.6 & 12.6 & 13.9 & 13.0 & 14.0 & 13.7 & 13.7 & 13.1 & 11.7 & 13.5 & 13.6 & 11.3 & 13.4 & 13.0 \\
\hline & 0.5 & 1.0 & 0.3 & 0.7 & 0.0 & 0.5 & 0.5 & 0.7 & 0. & 0.5 & 0.7 & 1.5 & 0. & 0.0 \\
\hline ISO & 0.5 & 0. & 0. & 0. & 0. & 0.5 & 0. & 0. & 0. & 0.5 & 0.5 & 0.5 & 0. & 0.5 \\
\hline & 0.0 & 0.0 & 0.0 & 0.0 & 0.0 & 0.0 & 0.0 & 0.0 & 0.0 & 0.0 & 0.0 & 0.0 & 0. & 0.0 \\
\hline MXTWP & 33.6 & 30.3 & 35.0 & 32.4 & 34.3 & 34.2 & 34.9 & 32.1 & 28.2 & 33.8 & 35.1 & 27.6 & 33.5 & 30.1 \\
\hline & 1.3 & 2.1 & 1.3 & 2.1 & 0.1 & 1.3 & 1.0 & 1.8 & 1.5 & 1.7 & 2.2 & 2.0 & 1.5 & 0.9 \\
\hline MNTCP & 5.0 & 5. & 5. & 5. & 4. & 5.4 & 5. & 4. & 5. & 6.4 & 5.6 & 5.8 & 5.3 & 5.1 \\
\hline & 0.5 & & 0. & 0.6 & 0.2 & 0.8 & 0.5 & 0.5 & 0.4 & 1.3 & 0.2 & 1.2 & 0.6 & 0.2 \\
\hline TAR & 28.6 & 25.3 & 29.4 & 27.0 & 30.0 & 28.9 & 29.3 & 27.2 & 22.7 & 27.5 & 29.4 & 21.9 & 28.1 & 25.0 \\
\hline & 1.1 & 2.5 & 0.9 & 1.8 & 0.1 & 0.7 & 0.8 & 1.8 & 2.0 & 2.0 & 2.1 & 3.1 & 1.4 & 1.2 \\
\hline MTDQ & 22.8 & 21.1 & 22.9 & 22.2 & 23.6 & 23.1 & 22.9 & 22.0 & 20.1 & 23.5 & 21.9 & 20.0 & 22.9 & 21.1 \\
\hline & 0.8 & 1.3 & 1.1 & 1.0 & & 0.6 & 0.9 & 1.3 & 0.7 & 1.2 & 1.1 & 0.1 & 0. & 0.9 \\
\hline MTWRQ & 24.3 & 21.6 & 25.5 & 23.2 & 25.1 & 24.8 & 25.6 & 23.0 & 20.4 & 24.7 & 25.8 & 20.5 & 24 & 21.5 \\
\hline & 1.2 & 1.5 & 1.2 & 1.9 & 0.1 & 1.4 & 0.9 & 1.6 & 0.7 & 1.5 & 1.8 & 0.6 & 1.3 & 0.6 \\
\hline MTCQ & 11.1 & 10.6 & 11.9 & 11.0 & 10.8 & 11.5 & 11.7 & 10.7 & 10.8 & 12.4 & 11 & 11.4 & 11.3 & 10.8 \\
\hline & 0.6 & 0.3 & 0.7 & 0.9 & 0.1 & 0.8 & 0.6 & 0.5 & 0.3 & 1.3 & 0.5 & 0.8 & 0.5 & 0.3 \\
\hline PANN & 328.2 & 347.4 & 328.3 & 386.5 & 276.5 & 327.6 & 307.1 & 325.2 & 390.8 & 405.9 & 302.5 & 467.3 & 309.3 & 333.3 \\
\hline & 40.6 & 38.2 & 25.4 & 39.5 & 4.0 & 37.8 & 24.3 & 24.7 & 51.6 & 100.7 & 21.4 & 138.0 & 9.8 & 4.3 \\
\hline PWETP & 58.7 & 50.7 & 62.6 & 70.4 & 42.0 & 60.3 & 56.0 & 51. & 50.7 & & 53.4 & 64.0 & 52.7 & 42.8 \\
\hline & 12.8 & 6.5 & 8.7 & 9.4 & 1.2 & 11.7 & 8.1 & 6.7 & 4.5 & 34.9 & 2.0 & 27.8 & 7.6 & 4.5 \\
\hline PSEAS & 60.7 & 45.8 & 66.8 & 66.9 & 44.5 & 63.9 & 61.8 & 50.8 & 38.0 & 72. & 58.5 & 40.0 & 56.7 & 34.3 \\
\hline & 10.6 & 11.0 & 7.6 & 8.8 & 2.9 & 8.6 & 7.2 & 9.0 & 4.7 & 21.7 & 7.9 & 14. & 12.0 & 7.5 \\
\hline PWETQ & 154.3 & 140.8 & 161.3 & 187.9 & 113.0 & 157.3 & 145.6 & 139.5 & 145.0 & 210.7 & 138.6 & 181.3 & 140.6 & 120.3 \\
\hline & & 18.1 & 19.9 & & 1.2 & 26.9 & 19.3 & 16.2 & 18.0 & 79.0 & 2.5 & 73.3 & 16.9 & 9.9 \\
\hline PWRMQ & 39.3 & 47.4 & 40.5 & 39.1 & 44.0 & 40.3 & 38.6 & 43.5 & 58.0 & 41.0 & 40.0 & 65.3 & 38.6 & 52.0 \\
\hline & 3.9 & 8.7 & 6.3 & 4.3 & 2.3 & 6.2 & 3.6 & 5.2 & 7.5 & 6.0 & 5.2 & 4.7 & 5.1 & 3.8 \\
\hline PCLDQ & 151.5 & 137.3 & 157.1 & 184.6 & 110.5 & 153.9 & 141.3 & 136.5 & 141.2 & 209.2 & 132.8 & 179.7 & 136.7 & 119.0 \\
\hline & 31.1 & 16.7 & 21.1 & 22.6 & 1.7 & 28.0 & 19.6 & 15.7 & 16.1 & 80.1 & 2.4 & 74.8 & 16.4 & 9.8 \\
\hline ELCODE & 3.3 & 3.6 & 3.7 & 3.8 & 3.5 & 3.4 & 3.1 & 3.1 & 3.3 & 2.3 & 2.7 & 2.3 & 2.1 & 2.5 \\
\hline & 0.6 & 0.5 & 0.5 & 0.4 & 0.6 & 0.8 & 0.6 & 0.7 & 1.0 & 1.0 & 0.6 & 0.6 & 0.4 & 0.6 \\
\hline & 26 & 20 & 39 & 12 & 4 & 7 & 30 & 39 & 6 & 12 & 11 & 3 & 7 & 4 \\
\hline $\mathrm{N}^{* *}$ & & 19 & & 12 & & 5 & & 35 & & & & 2 & & 2 \\
\hline
\end{tabular}




\begin{tabular}{|c|c|c|c|c|c|c|c|c|c|c|c|c|c|}
\hline Group & 7 & 8.1 & 8.2 & 9.1 & 9.2 & 10 & 11 & 12 & 13 & 14 & 15 & 16 & 17 \\
\hline \multirow[t]{2}{*}{ SIPRICH } & 4.00 & 14.47 & 26.00 & 17.63 & 18.57 & 6.18 & 3.00 & 3.73 & 4.64 & 13.93 & 8.56 & 11.40 & 21.24 \\
\hline & 1.49 & 6.42 & 7.88 & 8.60 & 8.62 & 4.98 & 1.41 & 1.19 & 2.69 & 9.17 & 5.39 & 5.13 & 10.32 \\
\hline \multirow[t]{2}{*}{ EC } & $7+2.21$ & 340.38 & 111.65 & $133.29^{*}$ & 325.75 & $253.72^{*}$ & 137.25 & 171.78 & 334.88 & $88.66^{*}$ & $28.44^{*}$ & 26.46 & 29.25 \\
\hline & 500.84 & 320.97 & 87.65 & 204.86 & 341.77 & 230.32 & 166.52 & 175.74 & 281.73 & 185.19 & 27.34 & 21.81 & 42.05 \\
\hline \multirow[t]{2}{*}{ pH } & 8.54 & 7.87 & 6.58 & $7.58^{*}$ & 8.24 & $7.61^{*}$ & 8.50 & 8.01 & 8.24 & $6.93^{*}$ & $6.10^{*}$ & 6.20 & 6.76 \\
\hline & 1.23 & 0.91 & 0.73 & 0.90 & 0.66 & 0.91 & 0.57 & 0.71 & 0.90 & 0.95 & 0.98 & 0.46 & 1.12 \\
\hline \multirow[t]{2}{*}{$O C$} & 0.59 & 0.55 & 0.52 & $0.96^{*}$ & 1.17 & $0.98^{*}$ & 0.30 & 1.35 & 0.87 & $1.26^{*}$ & $1.22^{*}$ & 0.74 & 1.27 \\
\hline & 0.24 & 0.52 & 0.32 & 0.83 & 1.05 & 0.81 & 0.06 & 2.27 & 0.80 & 1.06 & 1.25 & 0.93 & 1.15 \\
\hline \multirow[t]{2}{*}{$\mathrm{N}$} & 0.04 & 0.04 & 0.03 & $0.06^{*}$ & 0.10 & $0.07^{*}$ & 0.03 & 0.12 & 0.07 & $0.08^{*}$ & $0.07^{*}$ & 0.06 & 0.08 \\
\hline & 0.02 & 0.05 & 0.01 & 0.06 & 0.10 & 0.05 & 0.01 & 0.22 & 0.07 & 0.07 & 0.06 & 0.06 & 0.08 \\
\hline P & 86.40 & 92.80 & 45.92 & $74.13^{*}$ & 127.71 & $67.75^{*}$ & 180.00 & 138.91 & 76.18 & $84.48^{*}$ & $58.00^{*}$ & 276.00 & 111.63 \\
\hline & 25.37 & 100.76 & 25.42 & 98.41 & 134.30 & 41.95 & 84.85 & 88.02 & 42.86 & 71.59 & 51.17 & 114.15 & 150.17 \\
\hline PAV & 13.40 & 9.82 & 2.42 & $6.85^{*}$ & 5.14 & $6.58^{*}$ & 16.50 & 22.75 & 5.82 & $12.34^{*}$ & $3.38^{*}$ & 26.20 & 18.06 \\
\hline & 14.13 & 14.91 & 3.12 & 14.47 & 4.62 & 6.37 & 6.36 & 19.44 & 5.29 & 29.11 & 2.39 & 24.26 & 68.15 \\
\hline KAV & 496.00 & 375.40 & 120.27 & $219.25^{*}$ & 519.64 & $591.13^{*}$ & 1300.00 & 584.73 & 582.73 & $113,57^{*}$ & $46.25^{*}$ & 332.00 & 242.71 \\
\hline & 334.47 & 368.81 & 62.27 & 244.92 & 545.32 & 743.33 & 141.42 & 463.74 & 444.01 & 104.95 & 20.09 & 148.90 & 201.97 \\
\hline $\mathrm{Mg}$ & 4.19 & 3.10 & 1.36 & $2.95^{*}$ & 3.66 & $5.31^{*}$ & 5.72 & 3.55 & 5.30 & $3.33^{*}$ & $0.81^{*}$ & 2.20 & 2.17 \\
\hline & 2.72 & 2.57 & 0.76 & 2.70 & 2.79 & 3.93 & 2.89 & 2.96 & 3.61 & 3.38 & 0.53 & 1.33 & 1.75 \\
\hline $\mathrm{Ca}$ & 4.74 & 2.80 & 1.25 & $2.13^{*}$ & 4.88 & $2.35^{*}$ & 3.80 & 3.91 & 3.55 & $2.19^{*}$ & 1.07 & 3.02 & 3.91 \\
\hline & 3.43 & 3.03 & 1.76 & 1.87 & 3.79 & 1.62 & 0.26 & 4.31 & 3.08 & 2.35 & 1.36 & 3.77 & 2.25 \\
\hline $\mathrm{Na}$ & 6.01 & 2.37 & 0.85 & $1.92^{*}$ & 3.96 & $4.73^{*}$ & 6.18 & 3.28 & 4.84 & $1.66^{*}$ & 0.59 & 0.64 & 1.03 \\
\hline & 5.23 & 2.97 & 0.52 & 2.08 & 3.69 & 4.75 & 2.08 & 2.90 & 4.38 & 1.67 & 0.23 & 0.40 & 1.31 \\
\hline K & 1.15 & 0.92 & 0.27 & $0.61^{*}$ & 1.36 & $1.50^{*}$ & 3.88 & 1.59 & 1.37 & $0.23^{*}$ & 0.10 & 0.65 & 0.55 \\
\hline & 1.18 & 1.06 & 0.16 & 1.06 & 1.76 & 2.01 & 0.71 & 1.40 & 1.20 & 0.20 & 0.06 & 0.31 & 0.58 \\
\hline GYP & 52.70 & 7.15 & 1.86 & $0.05^{*}$ & 19.93 & $0.16^{*}$ & 0.00 & 0.19 & 0.23 & $0.00^{*}$ & 0.00 & 0.00 & 0.00 \\
\hline & 45.17 & 20.71 & 9.44 & 0.29 & 34.41 & 0.49 & 0.00 & 0.60 & 0.59 & 0.02 & 0.00 & 0.00 & 0.00 \\
\hline CLAY & $19.34^{*}$ & $6.54 *$ & $2.98^{*}$ & $4.78^{* *}$ & $5.66^{*}$ & $13.11^{*}$ & 27.05 & 10.23 & 19.62 & $3.49^{*}$ & 0.41 & 12.50 & 5.01 \\
\hline & 13.32 & 8.80 & 3.22 & 6.78 & 10.89 & 15.96 & 30.76 & 18.93 & 19.56 & 4.03 & 0.69 & 7.12 & 4.67 \\
\hline SILT & $4.29^{*}$ & $2.37^{*}$ & $1.53^{*}$ & $2.62^{* *}$ & $3.17^{*}$ & $4.25^{*}$ & 5.05 & 3.84 & 2.27 & $2.59^{*}$ & 1.35 & 5.60 & 4.06 \\
\hline & 2.98 & 2.55 & 0.93 & 2.44 & 2.34 & 3.68 & 3.18 & 2.69 & 2.24 & 2.34 & 0.76 & 2.84 & 3.47 \\
\hline SAND & $76.33^{*}$ & $91.10^{*}$ & $95.49^{*}$ & $92.61^{* *}$ & $91.18^{*}$ & $82.65^{*}$ & 67.90 & 85.93 & 78.11 & $93.93^{*}$ & 98.23 & 81.94 & 90.91 \\
\hline & 13.12 & 10.00 & 3.83 & 7.65 & 11.39 & 17.60 & 27.44 & 20.37 & 19.95 & 5.86 & 1.29 & 9.91 & 7.25 \\
\hline Long & 118.15 & 116.70 & 117.71 & 117.78 & 118.76 & 117.84 & 119.05 & 117.68 & 118.14 & 117.22 & 117.44 & 115.88 & 117.22 \\
\hline & 2.25 & 1.13 & 1.11 & 0.89 & 2.30 & 1.44 & 0.32 & 1.61 & 2.14 & 1.55 & 0.41 & 0.88 & 1.94 \\
\hline Lat & -30.89 & -30.23 & -31.09 & -32.72 & -32.83 & -32.70 & -32.66 & -31.74 & -32.10 & -32.23 & -32.24 & -28.62 & -31.15 \\
\hline & 1.97 & 1.40 & 1.17 & 1.05 & 1.49 & 1.47 & 0.98 & 1.79 & 1.50 & 1.67 & 0.82 & 0.91 & 1.90 \\
\hline Elev & 286.9 & 272.5 & 290.0 & 273.9 & 196.5 & 208.5 & 357.5 & 268.9 & 232.7 & 241.6 & 271.0 & 242.0 & 236.8 \\
\hline & 34.6 & 38.7 & 32.8 & 48.3 & 115.8 & 107.2 & 85.6 & 57.8 & 72.1 & 61.5 & 45.2 & 33.7 & 116.7 \\
\hline MTA & 17.9 & 18.5 & 17.8 & 16.2 & 16.4 & 16.5 & 16.1 & 17.1 & 16.9 & 16.5 & 16.7 & 19.8 & 17.6 \\
\hline & 1.7 & 1.2 & 1.1 & 1.0 & 1.5 & 1.1 & 0.6 & 1.4 & 1.2 & 1.3 & 1.0 & 0.3 & 1.7 \\
\hline MTD & 13.4 & 13.6 & 13.7 & 12.8 & 11.9 & 12.3 & 13.0 & 13.0 & 12.7 & 12.6 & 13.6 & 14.0 & 13.0 \\
\hline & 0.8 & 0.6 & 0.5 & 0.8 & 1.0 & 1.3 & 1.4 & 1.0 & 1.3 & 0.9 & 0.9 & 0.0 & 0.7 \\
\hline 150 & 0.5 & 0.5 & 0.5 & 0.5 & 0.5 & 0.5 & 0.5 & 0.5 & 0.5 & 0.5 & 0.5 & 0.5 & 0.5 \\
\hline & 0.0 & 0.0 & 0.0 & 0.0 & 0.0 & 0.0 & 0.0 & 0.0 & 0.0 & 0.0 & 0.0 & 0.0 & 0.0 \\
\hline MXTWP & 33.4 & 34.6 & 34.0 & 31.2 & 29.5 & 30.6 & 31.1 & 32.2 & 31.6 & 31.3 & 32.5 & 35.9 & 32.5 \\
\hline & 3.2 & 1.8 & 1.5 & 1.9 & 2.4 & 2.6 & 2.0 & 2.6 & 3.2 & 2.2 & 1.7 & 0.8 & 2.3 \\
\hline MNTCP & 5.5 & 5.8 & 5.1 & 5.1 & 6.0 & 5.7 & 4.5 & 5.4 & 5.6 & 5.5 & 4.9 & 6.4 & 6.1 \\
\hline & 0.4 & 0.7 & 0.7 & 0.5 & 1.4 & 1.0 & 0.7 & 0.8 & 0.6 & 1.0 & 0.2 & 0.8 & 1.5 \\
\hline TAR & 27.9 & 28.8 & 28.9 & 26.1 & 23.5 & 24.9 & 26.7 & 26.8 & 26.0 & 25.8 & 27.6 & 29.5 & 26.4 \\
\hline & 2.9 & 1.7 & 1.1 & 2.0 & 2.3 & 3.1 & 2.6 & 2.8 & 3.5 & 2.4 & 1.7 & 1.6 & 2.0 \\
\hline MTDQ & 21.3 & 22.8 & 22.9 & 21.5 & 21.1 & 21.6 & 21.4 & 22.1 & 21.8 & 21.5 & 21.9 & 21.8 & 22.2 \\
\hline & 1.3 & 1.2 & 1.0 & 1.0 & 1.8 & 1.4 & 0.4 & 1.4 & 1.6 & 1.4 & 0.8 & 0.3 & 1.4 \\
\hline MTWRQ & 24.4 & 25.3 & 24.7 & 22.2 & 21.5 & 22.1 & 22.2 & 23.4 & 22.9 & 22.5 & 23.2 & 26.8 & 23.7 \\
\hline & 2.8 & 1.6 & 1.4 & 1.6 & 1.9 & 1.8 & 1.5 & 2.1 & 2.3 & 1.8 & 1.5 & 0.3 & 2.1 \\
\hline MTCQ & 11.6 & 11.9 & 11.3 & 10.6 & 11.5 & 11.2 & 10.2 & 11.2 & 11.3 & 11.0 & 10.6 & 12.9 & 11.9 \\
\hline & 0.8 & 0.8 & 0.7 & 0.6 & 1.5 & 1.0 & 0.3 & 0.9 & 0.6 & 1.1 & 0.5 & 0.7 & 1.7 \\
\hline PANN & 317.0 & 333.7 & 314.8 & 382.7 & 430.1 & 439.7 & 344.0 & 382.1 & 416.6 & 449.3 & 363.3 & 282.8 & 384.3 \\
\hline & 15.0 & 33.5 & 23.0 & 49.9 & 102.5 & 87.9 & 11.3 & 148.7 & 129.7 & 93.4 & 51.9 & 9.4 & 74.5 \\
\hline PWETP & 51.6 & 63.6 & 55.4 & 64.4 & 71.3 & 73.3 & 52.0 & 65.7 & 71.0 & 81.6 & 65.3 & 56.0 & 73.3 \\
\hline & 9.2 & 11.7 & 7.7 & 11.6 & 35.0 & 18.7 & 1.4 & 25.9 & 20.9 & 20.5 & 7.7 & 3.7 & 29.2 \\
\hline PSEAS & 51.1 & 66.8 & 59.7 & 59.0 & 53.4 & 60.6 & 46.5 & 60.5 & 60.9 & 68.4 & 65.9 & 68.2 & 66.1 \\
\hline & 17.6 & 10.6 & 8.9 & 10.8 & 21.6 & 15.7 & 0.7 & 12.3 & 13.1 & 14.0 & 5.7 & 8.0 & 22.1 \\
\hline PWETQ & 135.8 & 164.0 & 146.2 & 174.9 & 190.8 & 199.8 & 142.0 & 176.2 & 190.6 & 218.9 & 175.6 & 140.0 & 189.0 \\
\hline & 18.6 & 26.6 & 18.4 & 29.7 & 78.9 & 49.2 & 2.8 & 69.3 & 56.9 & 50.6 & 22.1 & 4.0 & 63.6 \\
\hline PWRMQ & 47.5 & 40.8 & 38.7 & 41.9 & 50.3 & 48.2 & 48.5 & 43.7 & 47.5 & 41.8 & 36.1 & 42.4 & 41.1 \\
\hline & 8.6 & 54 & 3.9 & 7.0 & 11.7 & 15.0 & 2.1 & 12.1 & 13.1 & 11.1 & 5.1 & 4.3 & 9.1 \\
\hline PCLDQ & 131.4 & 159.8 & 142.7 & 171.3 & 188.3 & 196.4 & 139.0 & 172.6 & 188.7 & 216.4 & 171.8 & 133.2 & 185,9 \\
\hline & 17.2 & 27.1 & 18.2 & 30.0 & 79.7 & 50.6 & 0.0 & 69.9 & 58.4 & 52.0 & 23.1 & 4.0 & 64.5 \\
\hline ELCODE & 1.9 & 2.2 & 2.4 & 2.2 & 2.4 & 1.6 & 1.0 & 1.7 & 1.5 & 1.9 & 1.9 & 1.6 & 1.9 \\
\hline & 0.3 & 0.4 & 0.5 & 0.5 & 0.6 & 0.5 & 0.0 & 0.5 & 0.5 & 0.3 & 0.6 & 0.5 & 0.5 \\
\hline$N$ & 10 & 60 & 33 & 93 & 28 & 17 & 2 & 11 & 11 & 43 & 9 & 5 & 38 \\
\hline$N^{*}$ & 5 & 50 & 31 & 91 & 21 & 16 & & & & 42 & 8 & & \\
\hline$N^{* *}$ & & & & 89 & & & & & & & & & \\
\hline
\end{tabular}


Table 4 (cont.)

\begin{tabular}{|c|c|c|c|c|c|c|c|c|c|c|c|c|c|}
\hline Group & 18 & 19 & 20.1 & 20.2 & 21 & 22.1 & 22.2 & 23 & 24 & 25.1 & 25.2 & 26 & $\begin{array}{c}\text { All } \\
\text { Grps }\end{array}$ \\
\hline \multirow[t]{2}{*}{ SPPRICH } & 10.33 & 21.90 & 27.75 & 37.00 & 5.24 & 7.00 & 9.09 & 10.67 & 5.00 & 5.24 & 22.42 & 3.50 & 20.62 \\
\hline & 5.13 & 11.97 & 15.36 & 12.12 & 3.01 & 3.35 & 4.64 & 4.62 & 4.76 & 3.74 & 12.22 & 1.99 & 14.22 \\
\hline \multirow[t]{2}{*}{ EC } & 7.67 & $36.18^{*}$ & 15.33 & 9.35 & $48.01^{*}$ & 719.84 & 131.35 & 73.72 & $72.18^{*}$ & $67.65^{*}$ & 37.82 & $36.59^{*}$ & 137.81 \\
\hline & 4.04 & 57.31 & 13.35 & 6.48 & 66.25 & 916.97 & 225.47 & 96.46 & 108.29 & 71.42 & 65.61 & 23.65 & 247.90 \\
\hline \multirow[t]{2}{*}{$\mathrm{pH}$} & 6.20 & $6.00^{*}$ & 6.09 & 6.22 & $5.99^{*}$ & 8.18 & 6.81 & $5.75^{*}$ & $6.67^{*}$ & $7.00^{*}$ & 7.20 & $7.20^{*}$ & 7.07 \\
\hline & 0.40 & 0.59 & 0.52 & 0.40 & 0.88 & 0.82 & 1.26 & 0.21 & 1.46 & 1.06 & 1.28 & 0.84 & 1.13 \\
\hline \multirow[t]{2}{*}{$\mathrm{OC}$} & 0.91 & $4.72^{*}$ & 1.90 & 1.42 & $6.63^{*}$ & 3.38 & 3.20 & $3.66^{*}$ & $2.42^{*}$ & $2.36^{*}$ & 1.14 & $1.23^{*}$ & 1.28 \\
\hline & 0.74 & 4.22 & 1.68 & 1.48 & 9.22 & 2.22 & 7.04 & 0.34 & 3.62 & 2.67 & 0.90 & 1.02 & 2.23 \\
\hline \multirow[t]{2}{*}{$\mathrm{N}$} & 0.03 & $0.28^{*}$ & 0.12 & 0.12 & $0.40^{*}$ & 0.27 & 0.17 & $0.20^{*}$ & $0.18^{*}$ & $0.15^{*}$ & 0.06 & $0.08^{*}$ & 0.08 \\
\hline & 0.02 & 0.27 & 0.10 & 0.17 & 0.47 & 0.14 & 0.34 & 0.00 & 0.28 & 0.19 & 0.05 & 0.07 & 0.13 \\
\hline $\mathrm{P}$ & 15.00 & $142.37^{*}$ & 83.19 & 45.45 & $90.44^{*}$ & 355.91 & 250.73 & $53.50^{*}$ & $366.67^{*}$ & $58.35^{*}$ & 37.98 & $98.40^{*}$ & 87.36 \\
\hline & 12.12 & 114.96 & 53.72 & 55.90 & 71.58 & 280.97 & 473.48 & 0.71 & 453.25 & 69.55 & 26.73 & 69.05 & 114.43 \\
\hline PAV & 0.80 & $4.49^{*}$ & 3.41 & 1.76 & $4.28^{*}$ & 12.45 & 7.27 & $3.00^{*}$ & $35.67^{*}$ & $4.45^{*}$ & 2.35 & $13.32^{*}$ & 6.93 \\
\hline & 1.04 & 4.21 & 4.21 & 0.83 & 5.10 & 7.47 & 12.66 & 1.41 & 54.00 & 6.82 & 1.77 & 19.68 & 19.58 \\
\hline KAV & 53.00 & $131.68^{*}$ & 257.63 & 107.82 & $373.63^{*}$ & 610.00 & 195.09 & $325.00^{*}$ & $263.33^{*}$ & $289.20^{*}$ & 253.48 & $506.00^{*}$ & 275.39 \\
\hline & 41.22 & 95.30 & 246.79 & 96.80 & 313.47 & 374.70 & 276.24 & 134.35 & 274.29 & 390.20 & 533.30 & 363.63 & 363.72 \\
\hline $\mathrm{Mg}$ & 0.62 & $4.65^{*}$ & 3.41 & 1.59 & $6.25^{*}$ & 9.03 & 5.19 & $7.48^{*}$ & $3.05^{*}$ & $4.48^{*}$ & 1.58 & $3.46^{*}$ & 2.79 \\
\hline & 0.88 & 4.49 & 3.43 & 1.88 & 4.76 & 9.17 & 9.85 & 5.81 & 3.98 & 4.28 & 1.41 & 2.33 & 3.26 \\
\hline $\mathrm{Ca}$ & 1.12 & $4.72^{*}$ & 2.82 & 1.73 & $7.27^{*}$ & 4.31 & 3.89 & $6.01^{*}$ & $4.55^{*}$ & $4.18^{*}$ & 2.84 & $4.86^{*}$ & 3.13 \\
\hline & 0.34 & 3.96 & 1.64 & 1.30 & 5.60 & 2.63 & 6.79 & 3.38 & 6.71 & 3.80 & 2.74 & 2.91 & 3.43 \\
\hline $\mathrm{Na}$ & 0.24 & $1.43^{*}$ & 1.19 & 0.51 & $2.06^{*}$ & 13.35 & 3.04 & $1.59^{*}$ & $2.63^{*}$ & $2.23^{*}$ & 1.09 & $1.73^{*}$ & 1.93 \\
\hline & 0.36 & 1.78 & 1.46 & 0.50 & 1.80 & 19.21 & 5.14 & 0.40 & 3.76 & 2.25 & 1.93 & 1.05 & 3.61 \\
\hline $\mathrm{K}$ & 0.11 & $0.23^{*}$ & 0.64 & 0.20 & $0.71^{*}$ & 1.25 & 0.37 & 0.63 & $0.58^{*}$ & $0.78^{*}$ & 0.54 & $1.39^{*}$ & 0.68 \\
\hline & 0.09 & 0.15 & 0.86 & 0.18 & 0.65 & 0.94 & 0.48 & 0.33 & 0.64 & 1.56 & 1.34 & 1.08 & 1.13 \\
\hline GYP & 0.00 & $0.00^{*}$ & 0.00 & 0.00 & $0.00^{*}$ & 2.55 & 0.55 & 0.00 & $0.00^{*}$ & $0.01^{*}$ & 0.04 & $0.00^{*}$ & 3.75 \\
\hline & 0.00 & 0.00 & 0.00 & 0.00 & 0.00 & 5.50 & 1.29 & 0.00 & 0.00 & 0.02 & 0.29 & 0.00 & 16.51 \\
\hline CLAY & 3.06 & $3.58^{*}$ & 10.71 & 4.26 & $8.59^{* *}$ & $3.10^{*}$ & $2.54^{*}$ & 7.60 & $3.87^{*}$ & $5.20^{*}$ & 1.59 & $9.91^{*}$ & 5.24 \\
\hline & 1.70 & 4.11 & 5.22 & 3.29 & 7.08 & 4.34 & 2.39 & 9.48 & 0.25 & 10.59 & 1.73 & 7.70 & 8.22 \\
\hline SILT & 1.17 & $4.76^{*}$ & 6.33 & 3.41 & $5.34^{* *}$ & $3.83^{*}$ & $2.13^{*}$ & 3.70 & $3.40^{*}$ & $2.73^{*}$ & 1.82 & $4.29^{*}$ & 2.86 \\
\hline & 0.73 & 5.39 & 2.76 & 3.12 & 3.99 & 3.13 & 3.69 & 2.55 & 1.54 & 2.28 & 1.66 & 3.15 & 2.71 \\
\hline SAND & 95.77 & $91.69^{*}$ & 82.97 & 92.33 & $86.08^{* *}$ & $93.07^{*}$ & $95.33^{*}$ & $88.70^{*}$ & $92.73^{*}$ & $92.05^{*}$ & 96.59 & $85.75^{*}$ & 91.91 \\
\hline & 1.09 & 8.85 & 6.96 & 5.87 & 9.57 & 7.18 & 5.21 & 12.02 & 1.70 & 10.85 & 3.15 & 9.49 & 9.43 \\
\hline Long & 117.38 & 116.52 & 116.60 & 116.49 & 117.99 & 115.68 & 116.08 & 120.81 & 114.86 & 119.85 & 120.73 & 117.79 & 117.77 \\
\hline & 2.69 & 0.41 & 0.67 & 0.81 & 2.17 & 2.43 & 2.64 & 2.28 & 0.45 & 2.04 & 1.48 & 1.71 & 1.83 \\
\hline Lat & -31.43 & -32.84 & -32.71 & -32.66 & -34.03 & -29.50 & -30.02 & -34.26 & -28.66 & -34.05 & -33.92 & -32.27 & -31.90 \\
\hline & 2.92 & 1.36 & 1.69 & 1.64 & 0.46 & 2.05 & 2.57 & 0.49 & 0.42 & 0.35 & 0.28 & 1.61 & 1.81 \\
\hline Elev & 253.3 & 233.1 & 263.0 & 225.5 & 215.1 & 131.0 & 114.5 & 44.7 & 169.0 & 109.1 & 88.5 & 248.6 & 240.7 \\
\hline & 96.6 & 58.8 & 80.6 & 101.6 & 91.5 & 81.7 & 63.5 & 53.1 & 54.0 & 77.2 & 67.1 & 124.2 & 88.6 \\
\hline MTA & 17.0 & 15.9 & 15.9 & 16.0 & 14.9 & 18.7 & 18.4 & 15.6 & 19.2 & 15.6 & 15.9 & 16.7 & 17.0 \\
\hline & 2.0 & 1.3 & 1.6 & 1.9 & 0.4 & 1.2 & 1.3 & 0.6 & 0.2 & 0.7 & 0.5 & 1.8 & 1.5 \\
\hline MTD & 12.3 & 12.2 & 12.0 & 12.5 & 11.3 & 12.1 & 11.9 & 9.7 & 12.8 & 10.7 & 10.8 & 12.6 & 12.7 \\
\hline & 0.6 & 0.9 & 1.0 & 0.9 & 1.0 & 0.8 & 0.7 & 0.6 & 0.5 & 0.8 & 0.5 & 0.6 & 1.2 \\
\hline ISO & 0.5 & 0.5 & 0.5 & 0.5 & 0.5 & 0.5 & 0.5 & 0.6 & 0.5 & 0.5 & 0.5 & 0.5 & 0.5 \\
\hline & 0.0 & 0.0 & 0.0 & 0.0 & 0.0 & 0.0 & 0.0 & 0.0 & 0.0 & 0.0 & 0.0 & 0.0 & 0.0 \\
\hline MXTWP & 31.2 & 30.1 & 30.2 & 30.4 & 27.7 & 32.4 & 31.5 & 24.9 & 33.5 & 26.8 & 27.0 & 31.1 & 31.7 \\
\hline & 1.7 & 2.3 & 2.3 & 2.4 & 1.7 & 2.2 & 2.2 & 0.9 & 0.6 & 1.2 & 0.9 & 2.5 & 3.0 \\
\hline MNTCP & 5.9 & 5.6 & 5.8 & 5.6 & 5.5 & 7.6 & 7.8 & 7.1 & 7.6 & 6.4 & 6.5 & 5.8 & 5.6 \\
\hline & 1.9 & 0.9 & 1.0 & 1.6 & 0.9 & 1.3 & 0.7 & 0.1 & 0.7 & 0.6 & 0.5 & 1.5 & 1.0 \\
\hline TAR & 25.3 & 24.5 & 24.5 & 24.8 & 22.1 & 24.7 & 23.8 & 17.8 & 25.9 & 20.4 & 20.5 & 25.3 & 26.1 \\
\hline & 1.3 & 2.4 & 2.1 & 2.1 & 2.7 & 2.6 & 1.7 & 0.9 & 1.3 & 1.5 & 1.0 & 1.9 & 3.2 \\
\hline MTDQ & 21.9 & 21.5 & 21.0 & 21.1 & 19.7 & 22.4 & 22.3 & 19.2 & 23.0 & 19.8 & 20.1 & 21.8 & 21.8 \\
\hline & 0.9 & 1.9 & 1.6 & 2.0 & 0.7 & 0.8 & 1.2 & 0.7 & 0.4 & 0.7 & 0.5 & 1.8 & 1.5 \\
\hline MTWRQ & 22.7 & 21.5 & 21.6 & 21.7 & 19.8 & 24.4 & 23.7 & 19.5 & 25.3 & 19.9 & 20.1 & 22.4 & 23.0 \\
\hline & 2.1 & 1.8 & 2.1 & 2.2 & 0.6 & 1.5 & 2.0 & 0.8 & 0.2 & 0.6 & 0.5 & 2.3 & 2.4 \\
\hline MTCQ & 11.6 & 10.8 & 10.8 & 10.9 & 10.5 & 13.5 & 13.5 & 12.0 & 13.6 & 11.5 & 11.7 & 11.3 & 11.3 \\
\hline & 2.2 & 1.0 & 1.3 & 1.8 & 0.8 & 1.4 & 1.0 & 0.4 & 0.6 & 0.7 & 0.5 & 1.7 & 1.1 \\
\hline PANN & 369.7 & 676.5 & 568.5 & 619.6 & 644.9 & 419.4 & 460.0 & 683.3 & 424.8 & 561.0 & 523.9 & 412.1 & 406.2 \\
\hline & 45.0 & 104.8 & 96.0 & 132.1 & 151.8 & 72.2 & 81.4 & 30.0 & 8.6 & 95.7 & 58.8 & 93.5 & 118.7 \\
\hline PWETP & 68.0 & 127.4 & 103.6 & 119.3 & 105.5 & 93.6 & 96.5 & 103.7 & 100.3 & 80.4 & 70.7 & 72.8 & 70.3 \\
\hline & 29.6 & 19.4 & 23.0 & 30.7 & 32.3 & 19.4 & 32.2 & 4.0 & 5.2 & 21.0 & 11.4 & 34.8 & 24.9 \\
\hline PSEAS & 62.0 & 75.7 & 71.6 & 75.5 & 61.0 & 85.7 & 78.7 & 54.0 & 92.0 & 49.5 & 44.5 & 58.4 & 60.8 \\
\hline & 28.7 & 10.8 & 11.3 & 15.1 & 9.3 & 16.8 & 26.7 & 5.2 & 4.2 & 7.9 & 5.3 & 22.7 & 15.7 \\
\hline PWETQ & 177.7 & 342.4 & 281.9 & 316.1 & 291.7 & 234.0 & 243.3 & 287.0 & 247.3 & 228.0 & 202.6 & 191.3 & 188.0 \\
\hline & 63.4 & 49.4 & 58.2 & 77.1 & 83.7 & 40.5 & 69.9 & 6.9 & 9.0 & 53.5 & 31.0 & 77.1 & 63.6 \\
\hline PWRMQ & 42.0 & 48.8 & 45.8 & 44.9 & 58.2 & 35.7 & 43.9 & 74.0 & 33.8 & 66.6 & 69.5 & 45.1 & 45.5 \\
\hline & 7.8 & 13.5 & 9.5 & 10.6 & 14.1 & 10.5 & 17.5 & 8.7 & 2.1 & 8.4 & 5.1 & 9.6 & 12.0 \\
\hline PCLDQ & 172.3 & 342.1 & 281.1 & 315.3 & 291.2 & 226.8 & 236.9 & 286.3 & 241.8 & 226.1 & 200.7 & 188.9 & 184.8 \\
\hline & 59.1 & 49.4 & 59.2 & 78.2 & 83.8 & 37.9 & 71.0 & 8.1 & 6.9 & 54.7 & 32.5 & 78.5 & 64.6 \\
\hline ELCODE & 3.7 & 2.0 & 1.4 & 2.3 & 1.2 & 1.9 & 1.7 & 1.3 & 1.5 & 1.5 & 2.4 & 1.3 & 2.3 \\
\hline & 0.6 & 0.6 & 0.6 & 0.6 & 0.4 & 0.3 & 0.5 & 0.6 & 0.6 & 0.5 & 0.7 & 0.5 & 0.9 \\
\hline $\mathrm{N}$ & 3 & 21 & 16 & 11 & 17 & 11 & 11 & 3 & 4 & 21 & 48 & 14 & 760 \\
\hline $\mathrm{N}^{*}$ & & 19 & & & 16 & 9 & 10 & 2 & 3 & 20 & & 10 & \\
\hline$N^{* *}$ & & & & & 15 & & & & & & & & \\
\hline
\end{tabular}


Group 1.7 Mid-elevation chenopod shrublands. Group 1.7 was a group of 30 quadrats occurring at elevations intermediate between elevated dunes and the lower margins of saline lakes (mean ELCODE 3.07). These quadrats were largely chenopod-dominated shrublands and were rich in herbs. Taxa with high INDVAL scores include the shrubs Sclerolatna eurotioides, Maireana amoena, $M$. atkinsoniana and Didymanthus roei. Other species indicative of the group include Hyalochlamys globifer, Bromus arenarius and Triglochin minutissima. Mean species richness (36.2 taxa/quadrat) is comparable with non-chenopod-dominated shrublands and woodlands occurring on lunettes and rises (groups 1.2-1.4). Substrate EC is moderate $\left(68.8 \mathrm{mS} \mathrm{m}^{-1}\right)$ although the mean is elevated by a small number of quadrats with EC greater than 200 $\mathrm{mS} \mathrm{m} \mathrm{m}^{-1}$. The group includes two quadrats that were flooded with saline water during January 2000 and is largely found in the Avon 2 bioregion.

Group 1.8 Elevated chenopod shrublands on high $\mathrm{EC}$ sites. This group contained quadrats occupying similar elevations to group 1.7 but with higher mean soil EC, pH and exchangeable cations (Table 4). Gypsum was present at nine out of 39 quadrats. The quadrat group occurs in the Avon 2 and Mallee 2 bioregions, predominantly on the eastern sides of saline playas that have accumulated evaporites from the lakebed. These quadrats do not contain many of the herbs that define the preceding quadrat groups at similar elevations. High proportions of taxa per quadrat are introduced (mean $38 \%$ of taxa/ quadrat). Typical species (high INDVAL scores) include the shrubs Atriplex vesicaria and A. paludosa and the herbs, Podolepis tepperi and Pogonolepis muclleriana. A small number of quadrats were woodlands, dominated by Eucalyptus kondininensis

Group 1.9. Group 1.9 was a small group of quadrats from the Mallee 2 and Esperance Sandplains. This group represents a number of rarely sampled woodlands on elevated margins of saline lakes and playas.

Group 2 Inundated edges of northern basin wetlands. Group 2 was a heterogeneous group of near-coastal quadrats, occurring on the margins of northern brackish lakes and fresh sumps. Most were subject to inundation and were species poor. Various dominants occurred including Eucalyptus camaldulensis subsp. obtusa, Casuarina obesa, Melaleuca thuyoides and Melaleuca atroviridis ms.

Chenopod dominated berms, beaches and gypsum flats of natural and degraded primary saline wetlands

The second partition of the 12-group classification (35 quadrats) comprised Halosarcia and a few Melaleuca dominated shrublands occupying low elevations in saline wetlands. Quadrats were species poor and showed high EC, with gypsum commonly present (Table 4 ). They occurred almost exclusively in the Avon 2 and Mallee bioregions (Table 3).

Group 3 Northern species poor Halosarcia scrubs on gypsum substrates. Group 3 comprised 11 quadrats predominantly recorded from the Avon 2 bioregion (Table 3). Quadrats occupied low dunes, heaves and berms with high EC and pH (Table 4). Gypsum was present at nine quadrats and ranged from 11 to $97 \%$. A small number of taxa typify the group including Chondropyxis halophila, Rhodanthe heteranthera, Asteridea athrixioides and Atriplex holocarpa (Appendix 4). A group of northern quadrats occurred within the saline pans and playas on raised beds of almost pure gypsum (92-97\%). They were dominated by Halosarcia sp. Lake Moore (Lyons 2603) and contained Chondropyxis halophila, Frankenia conferta and Triglochin lyonsii ms (M.N. Lyons 2855). Quadrat group 3 includes a single disjunct quadrat in the Mallee 2 bioregion on a large lunette with high $\mathrm{pH}$ (but no gypsum) and fine textured soil.

Group 4 Melaleuca subalaris shrublands. Group 4 was a small group of three species poor quadrats (7.7 taxa/quadrat) sharing Halosarcia syncarpa, Wilsonia humilis and Melaleuca subalaris, occurring in the Mallee 1 and Esperance Sandplains 2 bioregions on the southeastern margin of the study area. They occurred on the edge of one coastal and two inland salt lakes. Melaleuca subalaris has a small natural range centred on the southeast of the study area. Mean EC and pH were high (Table 4).

Group 5 Species poor beaches of degraded saline wetlands. Group 5 was a group of seven species poor quadrats dominated by Melaleuca shrublands on bare beach edges with high substrate EC and very low $\mathrm{pH}$ (Table 4). These quadrats occur at the margins of degraded saline lakes (often acidic) at low elevations subject to inundation. They mostly occurred in the Avon 2 bioregion (Table 3). Typical species (high INDVAL scores) are Melaleuca thyoides, M. halmaturorum and Halosarcia lylei. A single very species poor quadrat on a sand dune in Frank Hann National Park is also grouped with these quadrats by the presence of Melaleuca thyoides.

Group 6 Gypseous berms of southern playas. Group 6 was a small group of four quadrats on the berms of southern playas with high mean EC and the presence of gypsum (Table 4). All quadrats occurred within the Mallee bioregion. Quadrats were species poor (mean 13 taxa/quadrat) and shared Sclerostegia moniliformis. Other taxa included Austrostipa juncifolia. Triglochin nanum subsp. salina $\mathrm{ms}$ and Matrana oppositifolia. A quadrat within a Callitris glaucophylla shrubland on low nongypseous sands within Lake King is included in the group by the presence of Sclerostegia moniliformis and Austrostipa juncifolia. These low Callitris dominated dunes were not sampled elsewhere

Group 7 Species poor Halosarcia halocnemoides 
gypsum flats. This group was comprised of 10 quadrats from the Avon Wheatbelt 2, Mallee 1 and Mallee 2 bioregions. Quadrats were species poor with high mean substrate $\mathrm{pH}$ and the highest mean EC of any quadrat group (Table 4). All quadrats contained Halosarcia halocnemoides and many were almost pure stands. They mostly occurred on gypseous flats (seven of ten quadrats) subject to inundation.

Species rich strands, berms and low sandy rises of naturally saline and degraded sub-saline/saline zetlands

The third block of the 12-group classification comprised a large and diverse group of 214 quadrats that occured at low elevations around a variety of saline wetlands. They were species rich relative to quadrats in the previous quadrat group (12-group level) particularly in herbs and chenopod shrubs. They were largely low chenopod dominated shrublands but included Melaleuca shrublands and Casuarina and Eucalyptus woodlands.

Group 8.1 Berms and beaches of saline pans and playas. Group 8.1 included a diverse group of quadrats from sandy and gypseous beaches, berms and low flats of primary saline playas and pans. The group shows high mean $\mathrm{EC}$ and $\mathrm{pH}$ relative to group 8.2 (Table 4) and contain variable amounts of gypsum $(0-99 \%)$. Quadrats share a widespread group of taxa that include Parapholis incurva, Sonchus oleraceus, Triglochin mucronata, Atriplex holocarpa and Cotula cotuloides. Taxa with high INDVAL scores included Halosarcia peltata, Triglochin mucronata and Gunniopsis septifraga (Appendix 4). Patterning within the group points to a subset of quadrats being degraded examples of more upslope vegetation (flooded/secondarily salinised) and the remainder being dominated by a group of berms, beaches and flats with variable gypsum content yet consistently high EC. Taxa such as Fitzwillia axilliflora, Neosciadium glochidiatum and Frankenia conferta are common on gypseous berms and beaches in the north of the study area.

Group 8.2 Sandy berms and low rises of saline pans and playas. Group 8.2 was comprised of quadrats on low, sandy berms and rises of primary saline playas and drainage lines. Quadrats occupy similar elevations to group 8.1, yet show lower mean substrate EC, pH and clay content. These quadrats display almost double the mean species richness of group 8.1 (26.0 cf. 14.5 taxa/quadrat). Typical species included Centrolepis humillima, Angianthus micropodioides, Centrolepis eremica, Gnephosis tridens and Atriplex hymenotheca. A distinctive subset of quadrats contains Sarcocornia globosa, Roycea pycnophylloides, Halosarcia sp. Central Wheatbelt (M.N. Lyons \& S.D. Lyons 2760) and Frankenia bracteata. Despite occurring in naturally saline systems, this group of quadrats is particularly threatened by increased flooding associated with dryland salinity.

Group 9.1 Margins of disturbed saline and subsaline wetlands. Group 9.1 was a large group of 93 quadrats, comprising low elevation margins of disturbed brackish, saline and secondarily saline wetlands. The group largely occurs within the Avon 1 and Mallee 2 bioregions and the western part of the Avon 2 bioregion. Quadrats have moderate mean substrate EC (Table 4). The group is defined by a number of widespread introduced taxa that occupy damp wetland margins and has an average of $42 \%$ introduced taxa per quadrat. Typical taxa (high INDVAL scores) include Spergularia marina, Parapholis incuroa and Hordeum marinum. Some of the taxa such as Polypogon monspeliensis, Juncus bufonius, Lythrum hyssopifolia, Pseudognaphalium luteoalbum and Vellereophyton dealbatum are widespread and also occur in fresh wetlands (see quadrat group 14). These occur in combination with annuals that are widespread within low elevation saline quadrats, such as Cotula bipinnata, Cotula coronopifolia and Triglochin mucronata. Canopy species include Casuarina obesa and Melaleuca halmaturorum in wooded margins and Halosarcia indica subsp. bidens, Sarcocomia quinqueflora and Halosarcia pergranulata in samphire margins.

Group 9.2 Gypseous berms and beaches of southern playas and pans and coastal salt lakes. Group 9.2 contained quadrats with high substrate $\mathrm{pH}$ and EC, that mostly contained gypsum (Table 4). Quadrats occurred at the margins (mean ELCODE of 2.4) of southern inland playas and pans, southern salt lakes and near coastal salt lakes. Inland occurrences were concentrated in the Mallee bioregion and coastal occurrences in the Esperance Sandplains and Geraldton Sandplains bioregions (Table 3). Typical taxa included Hydrocotyle medicaginoides, Brachyscome exilis, Halosarcia syncarpa and Isotoma scapigera. Other common species include Tegicornia uniflora, Austrostipa juncifolia, Goodenia sp. Lake King (M. Gustafsson et K. Bremer 132), Frankenia tetrapetala, Haegiela tatei and Vellea exigua.

\section{Mostly saline, inundated edges and species poor claypans.}

The fourth partition of the 12-group classification contained 41 quadrats that were very species poor. The quadrats sampled wetland basins and margins subject to inundation (ELCODE 1 and 2). Many quadrats were inundated during sampling and were distributed through most of the study area (Table 3).

Group 10 Inundated saline wetland margins. Group 10 was a heterogeneous group of species poor quadrats (6.2 taxa/quadrat) that were partially or shallowly inundated. Most are brackish/saline and typically include the aquatic Ruppia polycarpa. 
They occur in the higher rainfall parts of the study area predominantly in the Avon 1 bioregion. Other taxa with near coastal distributions are common including Wilsonia rotundifolia and $W$. backhousei. Quadrats included inundated Halosarcia pergranulata, Melaleuca teretifolia, $M$. rhaphiophylla, $M$. halmaturorum and $M$. cuticularis dominated riparian zones.

Group 11 Tecticornia verrucosa lake floors and claypans. Group 11 was a group of two very species poor quadrats ( 3 taxa/quadrat) that contained Tecticomia verrucosa. One quadrat included the highly geographically restricted Muehlenbeckia horrida subsp abdita.

Group 12 Group 12 was comprised of 11 very species poor quadrats (3.7 taxa/quadrat). They contained a small number of widespread taxa including Halosarcia indica subsp. bidens, Puccinellia stricta, Mesembryanthemum nodiflorum and Lolium spp. Quadrats on the beds of claypans within broad chenopod shrublands and containing Puccinellia stricta, Tecticomia verrucosa and Triglochin mucronata are subset of this group.

Group 13 Group 13 was a group of 11 very species poor quadrats (4.6 taxa/quadrat) that typically share Lepilaena preissii, Sarcocornia quinqueflora and Triglochin mucronata. Quadrats were partially or recently inundated margins of a variety of saline wetlands.

\section{Reservoirs, dams, saline seeps and disturbed freshwater} sites

The fifth partition of the 12-group classification contained 52 quadrats concentrated in the higher rainfall western Avon Wheatbelt and Jarrah Forest bioregions and coastal parts of the study area. Quadrats occurred in the basins and riparian zones of wetlands. Most quadrats were associated with semi-permanent and permanent water. This included seepage areas that were permanently wet.

Group 14 Disturbed wetlands with some saline influence. Group 14 contained quadrats from disturbed and artificial wetlands. The group included riparian margins of a diverse array of wetlands including creeks, dams, saline seeps, disturbed fresh and secondarily brackish/saline lake margins. Substrate EC is lower than groups 12 and 13, although very variable (Table 4). A suite of naturalized taxa including Cotula coronopifolia, Juncus acutus and Polypogon monspeliensis defines the group. On average half of the taxa per quadrat are introduced $(50.3 \%)$. The quadrats are concentrated in the higher rainfall Avon 1, western Avon 2 and Jarrah Forest bioregions (Table 3).

Group 15 Inundated dam and reservoir edges and basins. Group 15 was a group of nine quadrats similar to group 14 but occurring at non-saline wetlands. Quadrats sampled the inundated edges and basins of dams and reservoirs. They occurred in the Avon 2 and western Avon 1 bioregions (Table 4). Typical species (maximum INDVAL scores) were Symphyotrichum subulatum, Rumex crispus, Juncus pallidus and Typha spp. (Appendix 4).

\section{Inland claypans and herb-rich margins of freshwater wooded swamps}

The sixth block of the 12-group classification was a group of 43 quadrats from non-saline swamps and claypans scattered throughout the study area, with the exception of the high rainfall Jarrah Forest and Esperance Sandplains bioregions. Quadrats occurred in the basins (ELCODE 1) and margins (ELCODE 2) of wetlands with low substrate EC (Table 4).

Group 16 Northern claypan margins and basins. Group 16 was comprised of a small group of quadrats in the northeastern edge of the study area, occurring within and at the margins of non-saline claypans. They contained fewer species (11.4 taxa/ quadrat) than equivalent habitats in the south-west of the study area (27.8 and 37.0 taxa/quadrat, group 20.1 and 20.2 respectively). These herblands typically include Glossostigma drummondii, Myriocephalus gascoynensis, Calandrinia pumila, Isolepis congrua, Marsilea costulifera and Alternanthera nodiflora. Quadrats had low EC $\left(26.5 \mathrm{mS} \mathrm{m}^{-1}\right)$ and high clay and silt content $(12.5 \%$ and $5.6 \%$ respectively).

Group 17 Margins of wooded freshwater swamps - herb rich. Group 17 contained quadrats sampling the margins of freshwater wooded lakes, dams, swamps and claypans in lower rainfall areas. The group mostly occurs in the Avon 2, Mallee 2 and Geraldton Sandplains bioregions (Table 3). Quadrats showed low substrate EC $\left(29.3 \mathrm{mS} \mathrm{m}^{-1}\right)$. Melaleuca strobophylla was the most common canopy species. Northern quadrats were co-dominated by Eucalyptus camaldulensis var. obtusa and Eucalyptus loxophleba and southern quadrats by Eucalyptus occidentalis. Indicator species for these quadrats included Centipeda crateriformis subsp. crateriformis, Pseudognaphalium luteoalbum, Juncus subsecundus, Agrostis avenacea, Epilobium hirtigerum and Crassula decumbens. Elatine gratioloides and Crassula natans were also common. The inundated basins of these wetlands, that do not have this herbaceous component, are classified into quadrat groups 24 (northern) and 26 (central and southern).

\section{Rarely sampled shrublands and woodlands}

Group 18 A group of three species poor quadrats that shared Hypolaena exsulca comprised the seventh group of the 12-group classification.

\section{Southwestern claypans and riparian margins of non-} saline wetlands

The eighth group of the 12-group classification 
contained 65 relatively species rich quadrats from non-saline wetlands centred within the Jarrah Forest bioregion and immediately adjacent parts of the Avon Wheatbelt, Swan Coastal Plain, Esperance Sandplains and southern Geraldton Sandplains (Tables 3 and 4). They included the basins and riparian margins of sedge swamps, claypans, creeks and rivers.

Group 19 Riparian woodlands and shrublands of southwestern freshwater wetlands. Group 19 was a group of quadrats from high rainfall freshwater swamps, occurring largely within the Jarrah Forest 1 and 2 bioregions. Quadrats are riparian (mean ELCODE of 2.0) woodlands and shrublands that typically contain Lepidosperma longitudinale, Eucalyptus rudis, E. calophylla, Melaleuca preissiana, $M$. rhaphiophylla, Patersonia sp. Swamp form (N. Gibson and M. Lyons 544) and Deyeuxia quadriseta. Quadrats fringe sedge swamps and freshwater creeks. Many quadrats had dense sedge layers and few annuals.

Group 20.1 Southwestern claypan basins. Group 20.1 contained quadrats from herb rich shrublands and sedgelands in the basins of claypans within the higher rainfall south-west of the study area. A distinctive group of claypan specialists define this group of quadrats. They include Chorizandra enodis, Microtis orbicularis, Thelymitra aff. pauciflora, Myriocephalus occidentalis, Wurmbea dioica, Amphibromus nervosus and Meeboldina coangustata. Substrates were fine textured with very low EC (Table 4) and occurred within the Jarrah Forest and Swan Coastal Plain bioregions.

Group 20.2 Herb rich southwestern lake and claypan margins. This group was comprised of quadrats sampling herblands and shrublands on riparian flats of non-saline wetlands in the Jarrah Forest bioregion. Compared to quadrat group 20.1 these quadrats had coarser textured soils, were more elevated and species rich (Table 3 and 4). Typical species include Centrolepis aristata, Aphelia cyperoides, Stylidium calcaratum, Schoenus discifer, Velleia trinerois and Centrolepis mutica.

\section{Baumea sedge swamps}

Group 21 Baumea sedge swamps. Group 21 was comprised of species poor freshwater swamp basins containing Baumea articulata and Baumea arthrophylla. Other sedges include Baumea vaginalis and $B$. rubiginosa. Typical aquatics include Utricularia volubilis, Triglochin huegelii and Villarsia albiflora. Some quadrats included Melaleuca rhaphiophylla and Eucalyptus occidentalis as sparse canopy species. This group showed the highest mean soil organic carbon of all groups $(6.6 \%)$, with several quadrats occurring on pure peat. Quadrats have high annual precipitation and occurred in the Jarrah Forest, Esperance Sandplains and southern extremity of the Avon 1 bioregion (Table 3).
Coastal and southwestern saline and freshwater sedgelands and shrublands

The tenth group of quadrats of the 12-group classification was a somewhat heterogeneous group of 29 quadrats, associated with coastal wetlands that were mostly saline. Sedges and/or Juncus kraussii subsp. australiensis dominated most quadrats.

Group 22.1 Species poor margins of coastal lakes and lagoons. This group is largely defined by the presence of Juncus kraussii subsp. australiensis, Sarcocornia quinqueflora and Suaeda australis. Samolus repens and Sporobolus virginicus are also commonly present. Substrates showed very high mean EC (719 $\left.\mathrm{mS} \mathrm{m}^{-1}\right)$. The group includes three quadrats from a natural artesian fed saline spring near Dowerin. The permanent seepage at this wetland supports Juncus kraussii subsp. australiensis and Suaeda australis, taxa that are rarely recorded away from the coast.

Group 22.2 Miscellaneous near-coastal wetland edges with Juncus kraussii. Group 22.2 contained a near coastal group of springs, seeps and west flowing streams, closely related to group 22.1. They mostly contain Juncus krausii subsp. australiensis but include variously Melaleuca rhaphiophylla, Baumea juncea, Samolus junceus and Lobelia alata. Substrate $\mathrm{EC}$ is lower $\left(131 \mathrm{mS} \mathrm{m}^{-1} \mathrm{cf} .719 \mathrm{mS} \mathrm{m}^{-1}\right)$ than group 22.1 .

Group 23 South coast sedgelands. Group 23 was a small group of three sedgeland quadrats from the eastern south coast. Indicative taxa include Lepyrodia muirit, L. fortunata, Melaleuca incana subsp. tenella, Boronia denticulata and Gahnia trifida. They are more species rich than more deeply inundated quadrats dominated by Baumea articulata (cf. quadrat group 21, Table 4). These high rainfall sedgelands were not sampled elsewhere in the study area.

Group 24 Northern wooded freshwater wetland basins and margins. Group 24 was comprised of Eucalyptus camaldulensis var. obtusa wooded wetland basins and margins occurring in the Geraldton Sandplains 2 bioregion. Typically quadrats contained Cyperus gymnocaulos and Acacia saligna. This is a small group of 4 quadrats with low species richness (mean 5 taxa/quadrat). They cluster with groups 22 and 23 because a few quadrats in each share Juncus krausii subsp. australiensis and Eucalyptus camaldulensis var. obtusa.

\section{Southern near coastal lakes and swamps}

This group forms the eleventh block of quadrats of the 12-group classification and contains 69 quadrats from riparian zones and dunes of southern coastal, saline and brackish wetlands. They typically contain Melaleuca cuticularis.

Group 25.1 Southern near coastal lakes and swamps - species poor edges. This group is a species poor subset of group 25 (5.2 taxa/quadrat), 
typically dominated by Melalewa cuticularis (INDVAL of 95). Quadrats often included Baumea juncea and Gahnia trifida. Typically quadrats occur at the edge of coastal and near coastal saline and brackish wetlands.

Group 25.2 Southern near coastal lakes and swamps - species rich margins and elevated dunes. This group was comprised of quadrats that occupy riparian zones and dunes surrounding a variety of wetlands within the Esperance Sandplains bioregion. They typically contain Melaleuca cuticularis (INDVAL of 83). The group occurs at higher elevations than quadrat group 25.1 (mean ELCODE 2.4 cf. 1.5, Table 4). Other typical species include Isolepis nodosa. Acacia cyclops, Eucalyptus occidentalis and the weeds Hypochaeris glabra and Vulpia spp. Mean species richness is four times that of group 25.1 (Table 4). A subset of this group represents a small number of quadrats that intergrade in composition with quadrat group 26. They typically contain Melaleuca strobophylla, $M$. cuticularis and Eucalyptus occidentalis as dominants and occur close to the south coast.

\section{Yate and Melaleuca strobophylla basins}

Group 26 Melalenca strobophylla and Yate (Eucalyptus occidentalis) swamp basins. Group 26 was comprised of quadrats containing Melaleuca strobophylla, in combination with either Casuarina obesa or Eucalyptus occidentalis. Quadrats in the east may include Muehlenbeckia florulenta. Eragrostis australasica, a species rarely recorded within the study area, was present at four quadrats. Species richness is very low at $3.5 \mathrm{taxa} /$ quadrat. This group is the southern analogue of quadrat group 24 .

The quadrat groups showed strong geographic patterning across the study area (Table 3). There was however very little congruence between quadrat groups and the IBRA regions and subregions, or the distribution of quadrat groups within major catchments basins (Tables 3 and 5). Of the 39 quadrat groups, only group 6 was confined to a single IBRA region and only group 24 to a single sub-region. Groups 1.5 and 24 were confined to single catchments (Table 5).

Kruskal-Wallace non-parametric ANOVA showed significant differences $(P<0.0001)$ between groups at the 12, 26 and 39 group levels for all environmental attributes (groups with less than five quadrats excluded, Table 4). The three dimensional ordination (stress $=0.21$ ), showed significant linear correlations with 31 of the 34 environmental attributes measured (Table 6). Strongest correlations were found for the climate variables, annual temperature range, mean diurnal temperature and annual rainfall. Elevation code, substrate $\mathrm{pH}$ and EC showed the strongest correlations of the non-climatic parameters. The plot of ordination axis 1 versus 2 and 1 versus 3 , showed a group of rainfall related vectors running at $180^{\circ}$ to a group of temperature related vectors (Figure 8). Diverging from these were a subset of the edaphic variables, gypsum, EC, pH, K, KAV and $\mathrm{Na}$ (Figure 8a). Organic carbon and total nitrogen vectors paralleled the rainfall vectors on both ordination plots.

The plot of ordination axis 1 and 2 shows strong concentration of groups 2, 3 and 4 (at the 12-group level) in the upper left quadrant of the ordination. These three quadrat groups were chenopod dominated berms, beaches, low sandy flats and gypsum flats of varying species richness (see Figure 7). Strong EC, gypsum and $\mathrm{pH}$ gradients run toward this corner of the ordination (Figure $8 \mathrm{a}$ ). Quadrat groups (at the 12-group level) concentrated in the lower right quadrant of the ordination include Baumea sedge swamps (Group 9), southwestern claypans and riparian margins of freshwater swamps (Group 8) and southern near coastal lakes and swamps (Group 11). A strong annual rainfall gradient runs to the lower right quadrant (Figure 8a).

The plot of ordination axes 1 and 3 shows group 1 - woodlands and shrublands of lunettes, interwetland flats and rises in inland saline systems and elements of group 2 and 3, concentrated in the bottom left of the ordination (Figure $8 \mathrm{~b}$ ). A strong temperature gradient runs to this corner of the ordination. The vector for elevation (ELCODE) parallels the temperature gradient. This reflects these groups occurrence in elevated positions at inland wetlands.

\section{Species assemblages}

At higher levels in the species classification four broad partitions could be recognized (Figure 9). The primary division separated a large group of 331 taxa (group IV) with predominantly semi-arid and arid distributions from the remainder of the dataset (Figures 9 and 10). Few of these taxa (14.5\%) were restricted to wetland habitats although some were taxa specifically associated with inland salt lake systems (Table 7).

Groups II and III at the four-partition level contained taxa with distributions largely centred within the mesic south-west and coastal parts of the study area (Figures 9 and 10). Group III was dominated by herbaceous taxa, with approximately $43 \%$ of all taxa in the group restricted to wetland habitats (Table 7 ). The group occurred in southwestern non-saline wetlands (claypans, sedgelands and basins) with scattered inland occurrences.

Group II included a diverse suite of taxa, again occurring largely in the mesic south-west but extending into the semi-arid parts of the study area (Figures 9 and 10). The majority of taxa $(79 \%)$ were not restricted to wetland habitats. 

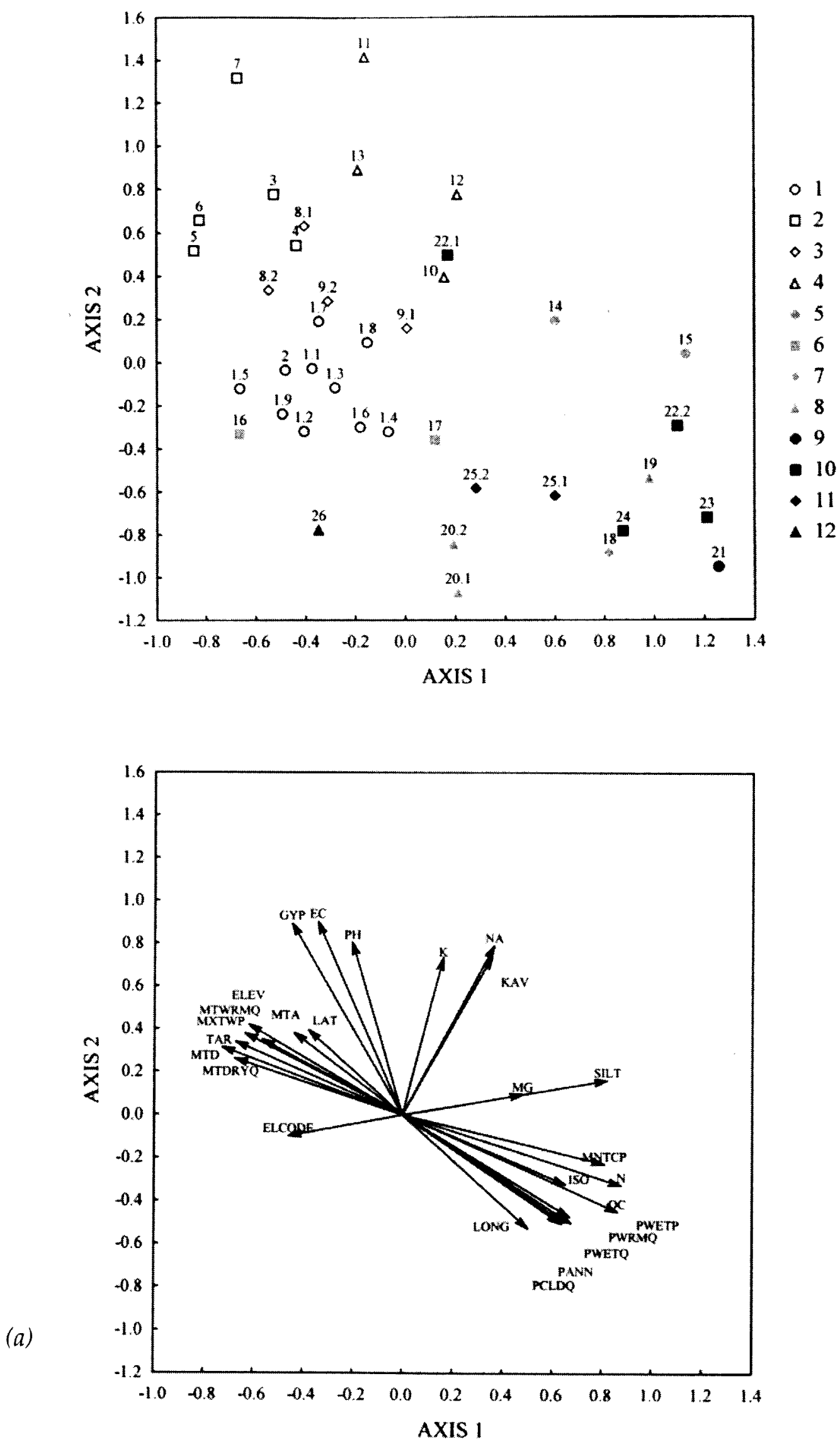

Figure 8 Ordinations showing group centroids for the 39 - group level (numbers) and 12 - group level (symbols) classifications. Significant principal axis correlations $(P<0.05)$ with environmental attributes are shown as vectors. See Table 1 and 6 for attribute abbreviations and correlations. (a) Axis 1 vs. Axis 2, (b) Axis 1 vs. Axis 3. 

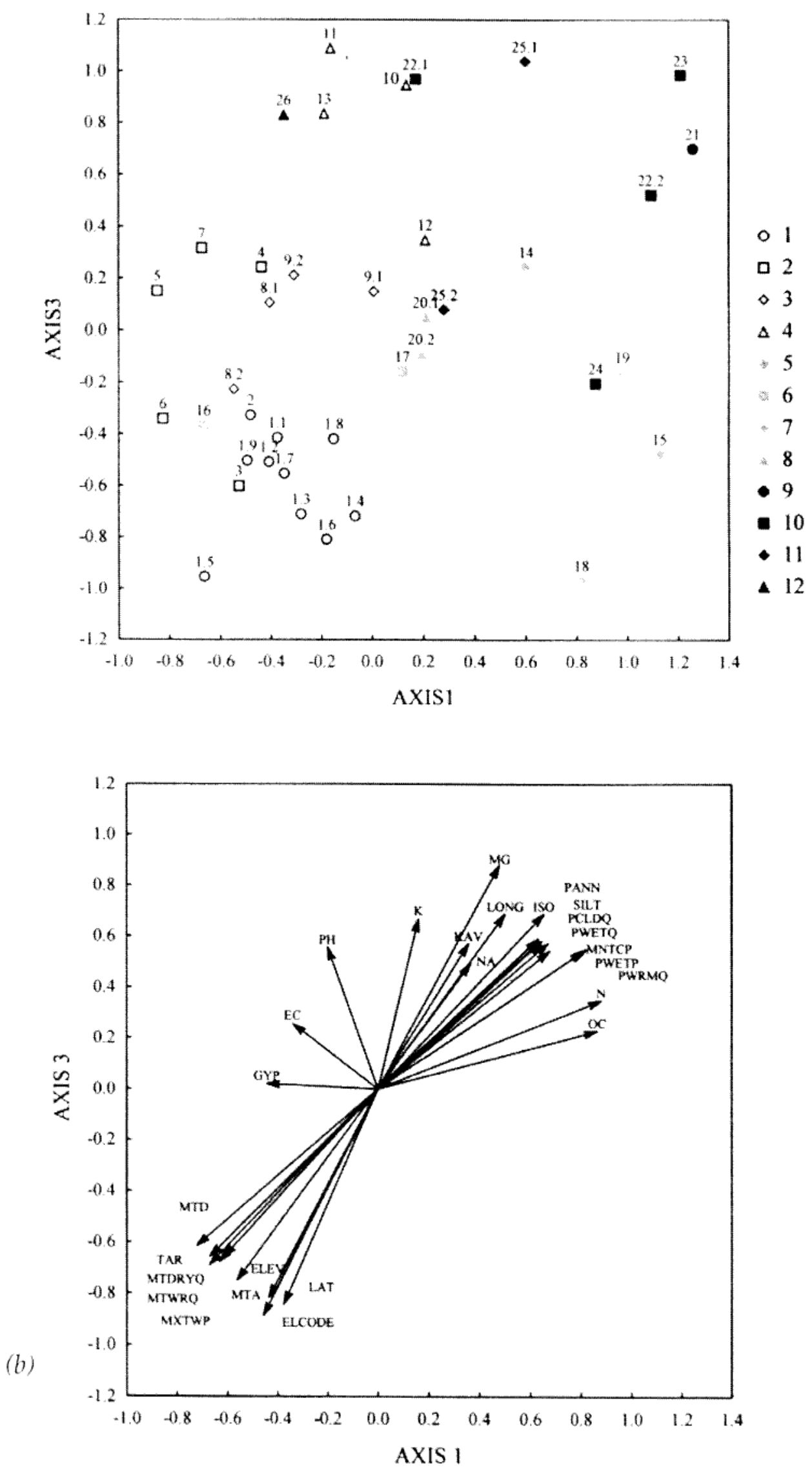
Table 5 Occurrence of 39 quadrat groups across major catchment basins. Y, Yarra Yarra; N, Ninghan; AV, Avon River; YI, Yilgran; LO, Lockhart; M, Murchison River; GR, Greenough River; MHR, Moore - Hill River; MR, Murray River; CR, Collie River; BR, Blackwood River; KR, Kent River; WR, Warren River; SR, Shannon River; FR, Frankland River; AC, Albany Coast; EC, Esperance Coast; SCP, Swan Coastal Plain; SLB, Salt Lake Basin (see Figure 2).

\begin{tabular}{|c|c|c|c|c|c|c|c|c|c|c|c|c|c|c|c|c|c|c|c|c|}
\hline $\begin{array}{l}\text { Site } \\
\text { group }\end{array}$ & $Y Y$ & $\mathbf{N}$ & $\mathrm{AV}$ & $\mathbf{Y I}$ & LO & MrR & GR & MHR & MR & $\mathrm{CR}$ & BR & $\mathrm{KR}$ & WR & SR & FR & $\mathrm{AC}$ & EC & SCP & SLB & $\mathrm{n}$ \\
\hline 1.1 & - & 2 & 6 & 6 & 7 & - & - & 5 & - & - & - & - & - & - & - & - & - & - & - & 26 \\
\hline 1.2 & - & - & 1 & 1 & 12 & - & - & - & - & - & - & - & - & - & - & 3 & 2 & - & 1 & 20 \\
\hline 1.3 & 11 & 2 & 3 & 1 & 6 & - & 7 & 9 & - & - & - & - & - & - & - & - & - & - & - & 39 \\
\hline 1.4 & - & - & 4 & - & 1 & - & - & 2 & - & - & 5 & - & - & - & - & - & - & - & - & 12 \\
\hline 1.5 & - & - & - & 4 & - & - & - & - & - & - & - & - & - & - & - & - & - & - & - & 4 \\
\hline 1.6 & - & - & 2 & 1 & 2 & - & 1 & 1 & - & - & - & - & - & - & - & - & - & - & - & 7 \\
\hline 1.7 & 10 & 3 & 9 & 5 & - & - & 1 & 2 & - & - & - & - & - & - & - & - & - & - & - & 30 \\
\hline 1.8 & 1 & - & 5 & 10 & 23 & - & - & - & - & - & - & - & - & - & - & - & - & - & - & 39 \\
\hline 1.9 & - & - & - & - & 2 & - & - & - & - & - & - & - & - & - & - & 3 & 1 & - & - & 6 \\
\hline 2 & - & - & 1 & - & - & - & 1 & 8 & - & - & - & - & - & - & - & - & - & - & 2 & 12 \\
\hline 3 & 5 & 5 & - & - & - & - & - & - & - & - & - & - & - & - & - & 1 & - & - & - & 11 \\
\hline 4 & - & - & - & - & - & - & - & - & - & - & - & - & - & - & - & - & 1 & - & 2 & 3 \\
\hline 5 & - & - & 2 & 2 & 2 & - & - & - & - & - & - & - & - & - & - & - & - & - & 1 & 7 \\
\hline 6 & - & - & - & - & 2 & - & - & - & - & - & - & - & - & - & - & - & 2 & - & - & 4 \\
\hline 7 & 1 & 2 & - & - & 2 & - & 2 & 1 & - & - & - & - & - & - & - & - & - & - & 2 & 10 \\
\hline 8.1 & 17 & 3 & 9 & 1 & 8 & - & 9 & 11 & 1 & - & - & - & - & - & - & 1 & - & - & - & 60 \\
\hline 8.2 & 6 & 2 & 6 & 6 & 10 & - & - & 3 & - & - & - & - & - & - & - & - & - & - & - & 33 \\
\hline 9.1 & - & - & 22 & 2 & 19 & - & - & 6 & - & - & 37 & - & - & - & - & 5 & 2 & - & - & 93 \\
\hline 9.2 & - & - & - & - & 7 & - & - & 5 & - & - & 1 & - & - & - & - & 8 & 4 & - & 3 & 28 \\
\hline 10 & - & - & 3 & - & 2 & - & 1 & - & - & - & 4 & - & - & - & 1 & 2 & 2 & 2 & - & 17 \\
\hline 11 & - & - & - & 1 & 1 & - & - & - & - & - & - & - & - & - & - & - & - & - & - & 2 \\
\hline 12 & 1 & - & 3 & 1 & 2 & - & - & - & 1 & - & 1 & - & - & 1 & - & - & 1 & - & - & 11 \\
\hline 13 & - & - & 3 & - & 3 & - & - & 2 & - & - & - & - & 1 & - & - & - & 2 & - & - & 11 \\
\hline 14 & - & - & 19 & - & - & 3 & 4 & 1 & - & - & 8 & 1 & - & 1 & 1 & 2 & 2 & - & 1 & 43 \\
\hline 15 & - & - & 4 & - & 3 & - & - & - & - & - & 2 & - & - & - & - & - & - & - & - & 9 \\
\hline 16 & 3 & - & - & - & - & - & - & - & 2 & - & - & - & - & - & - & - & - & - & - & 5 \\
\hline 17 & - & - & 7 & 4 & 10 & - & 5 & 6 & 3 & - & - & - & - & - & - & 2 & - & - & 1 & 38 \\
\hline 18 & - & - & - & - & 2 & - & 1 & - & - & - & - & - & - & - & - & - & - & - & - & 3 \\
\hline 19 & - & - & - & - & - & 1 & - & 1 & - & 1 & 2 & 1 & 4 & 2 & - & - & - & 9 & - & 21 \\
\hline 20.1 & - & - & 2 & - & - & - & 1 & 2 & - & - & 1 & - & 2 & 1 & 5 & - & - & 2 & - & 16 \\
\hline 20.2 & - & - & - & - & - & - & - & 2 & - & 2 & 1 & 1 & 1 & - & - & 1 & - & 3 & - & 11 \\
\hline 21 & - & - & - & - & - & - & - & - & - & 2 & 3 & 2 & 1 & 2 & 3 & 1 & 3 & - & - & 17 \\
\hline 22.1 & - & - & - & - & - & - & 3 & 1 & 1 & - & - & - & - & - & - & 2 & 1 & - & - & 8 \\
\hline 22.2 & - & - & 3 & - & - & - & 9 & 1 & - & - & - & - & - & - & - & - & 1 & - & - & 14 \\
\hline 23 & - & - & - & - & - & - & - & - & - & - & - & - & - & - & - & 1 & 2 & - & - & 3 \\
\hline 24 & - & - & - & - & - & - & 4 & - & - & - & - & - & - & - & - & - & - & - & - & 4 \\
\hline 25.1 & - & - & - & - & - & - & - & - & - & - & 1 & - & - & 2 & - & 6 & 12 & - & - & 21 \\
\hline 25.2 & - & - & - & - & 1 & - & - & - & - & - & - & 1 & - & - & - & 15 & 27 & - & 4 & 48 \\
\hline 26 & - & - & 2 & 2 & 4 & - & - & 3 & - & - & 1 & - & - & - & - & 2 & - & - & - & 14 \\
\hline
\end{tabular}

Group I at the four-group level was dominated by taxa restricted to wetland habitats (ca. 63\%) and included the majority of aquatic (20 of 34) and amphibious plants ( 37 of 71 ) within the dataset (Table 7). The group was comprised of taxa that occupied an array of specific wetland habitats (Figure 9).

Inspection of the species classification showed distinct and ecologically meaningful patterning occurring at low levels of the dendrogram (Figure 9). To reveal these groups the classification was partitioned into a total of 34 groups and subgroups. The fine level classification groups were regarded as species assemblages.
A feature of the classification was the presence of a number of assemblages with few members (10 taxa or less). A number of these groups (assemblages 1.1, 2.2, 2.9, 11 and 22) were almost exclusively composed of terrestrial taxa (Table 7). Since the current survey sampled a very limited suite of terrestrial habitats - i.e. only specific landforms associated with wetlands - these groups were regarded as poorly sampled and artifactual. Other assemblages with few taxa were dominated by wetland restricted taxa (assemblages 2.5, 2.6, 2.7, $2.8,4.2$ and 12, Table 7). These were regarded as coherent groups and not artifacts of limited sampling. They represented clusters of species 
Table 6 Principal axis correlations of environmental attributes with the three dimensional quadrat ordination. The significance of correlations was tested using 1000 Monte Carlo simulations $(*, P<0.05 ; * *, P<0.01 ; * * *, P<0.001$; NS, $P>0.05)$.

\begin{tabular}{|c|c|}
\hline TAR & 0.4319 \\
\hline MTD & 0.4087 \\
\hline PANN & 0.4066 \\
\hline ELCODE & 0.3864 \\
\hline 150 & 0.3832 \\
\hline MXTWI' & 0.3685 \\
\hline ELEV ${ }^{\cdots}$ & 0.3495 \\
\hline PWETQ & 0.3296 \\
\hline I'CLDQ' & 0.3296 \\
\hline I'H" & 0.3184 \\
\hline MTWRMQ & 0.3095 \\
\hline PWRMQ & 0.3019 \\
\hline PWETP & 0.2950 \\
\hline MNTCP" & 0.2764 \\
\hline MTDRYQ & 0.2493 \\
\hline $\mathrm{EC}^{\cdots}$ & 0.2283 \\
\hline GYP & 0.2206 \\
\hline LAT $^{* \cdot}$ & 0.2193 \\
\hline$N^{\cdots}$ & 0.2131 \\
\hline $\mathrm{OC}^{\cdots}$ & 0.2077 \\
\hline $\mathrm{MTA}^{\cdots}$ & 0.2065 \\
\hline $\mathrm{KAV}^{\cdots}$ & 0.1462 \\
\hline$M G^{\prime \prime}$ & 0.1451 \\
\hline NA & 0.1430 \\
\hline SILT & 0.1340 \\
\hline LONG" & 0.1290 \\
\hline$K^{\prime}$ & 0.1282 \\
\hline CLAY & 0.1242 \\
\hline SAND & 0.1206 \\
\hline$P^{\prime}$ & 0.1070 \\
\hline $\mathrm{CA}^{\mathrm{S}}$ & 0.1046 \\
\hline MTCQ & 0.1040 \\
\hline PAV & 0.1006 \\
\hline PSEAS '" & 0.0196 \\
\hline
\end{tabular}

occupying very specific wetland habitats (e.g. gypsum deposits, freshwater wetland basins, or leached sandy deposits in saline playas).

The species assemblages are described below. Major headings correspond to the four-partition level (see Figure 9).

\section{Species assemblages}

Assemblases of specific saline and non-saline zeetland habitats

Assemblage 1.1 An artifactual assemblage of very rarely recorded terrestrial taxa. The assemblage included eight taxa occurring in a total of eleven quadrats. The clustering is driven by the occurrence of four taxa in a single quadrat.

Assemblage 1.2 Taxa from inland primary salt lake systems and associated calcareous soils (lake parna). Several taxa are associated with gypsum substrates (e.g. Goodenin sp. Lake King (M Gustafsson \& K. Bremer 132)). The assemblage occurs in the Avon Wheatbelt, Mallee, Yalgoo and Coolgardie bioregions and extends into the adjacent arid zone. Taxa include Kippistin suacdifolin. Asteridea athrixioides, Hemichroa diandra and Sondottia comnata. The assemblage includes taxa distributed beyond immediate salt lake habitats on calcareous soils such as Eucalyptus flocktomiac and Maireana appressa (Appendix 4, Figure 10). Of the 43 taxa in the assemblage 14 are confined to wetland habitats (Table 7).

Assemblage 2.1 Taxa of freshwater wetland basins and margins. This group is almost exclusively native and largely distributed outside the higher rainfall areas of the South West. They mostly occupy wooded swamps and claypans but also occur within artificial waterbodies such as reservoirs and dams. The assemblage includes Persicaria prostrata, Elatine gratioloides, Marsilea costulifera, Centipeda crateriformis subsp. compacta, Persicaria prostrata and the aquatics Ottelia owalifolia, Lemma disperma and Lepilacma australis. Some species such as Cyperns gymmocaulos, Eragrostis australasica and Eucalyptus camaldulensis var. obtusa have a strongly northern distribution centred on the Geraldton Sandplains and adjacent regions of the arid zone (Figure 10, Appendix 4). Most of the component taxa (ca. $74 \%$, see Table 7 ) are known only from wetlands. A number of the remaining taxa, not restricted to wetlands, are introduced and include Hordeum geniculatum, Echium plantagineum, Conyza albida and Solanum nigrum.

Assemblage 2.2 An artifactual group of seven terrestrial species recorded almost exclusively from two quadrats.

Assemblage 2.3 An assemblage that includes the aquatics Lepilaena preissii, Lepilacha cylintrocarpa, Ruppia megacarpa and Ruppia polycarpa. Many saline and hypersaline quadrats containing these aquatic taxa were removed from the dataset. These aquatic taxa co-occur with a heterogeneous group of taxa that occur in inundated samphire shrublands, lake basins and some claypans. The assemblage therefore includes inland claypan taxa such as Tectionmia erracosa and Muehlenbeckin horrida subsp. abdita. Also included are a small group of taxa known from coastal saline wetlands, including Apium prostratum, Triglochin striatum and Hemichroa pentantra (Appendix 4, Figure 10).

Assemblage 2.4 Taxa of freshwater wetland basins and sedge swamps in the high rainfall and near coastal parts of the south-west. Most taxa have distributions centred on the Swan Coastal Plain, Warren and Jarrah Forest bioregions. Component taxa are almost exclusively aquatic or amphibious and include Bamea articulata, B. arginalis, B. rubiginosa and B. arthrophylla. Aquatic members of the assemblage include Triglochin hacgelit Utricularia arolubilis and Potamogeton drammontil (Figure 10, Table 7, Appendix 4). 


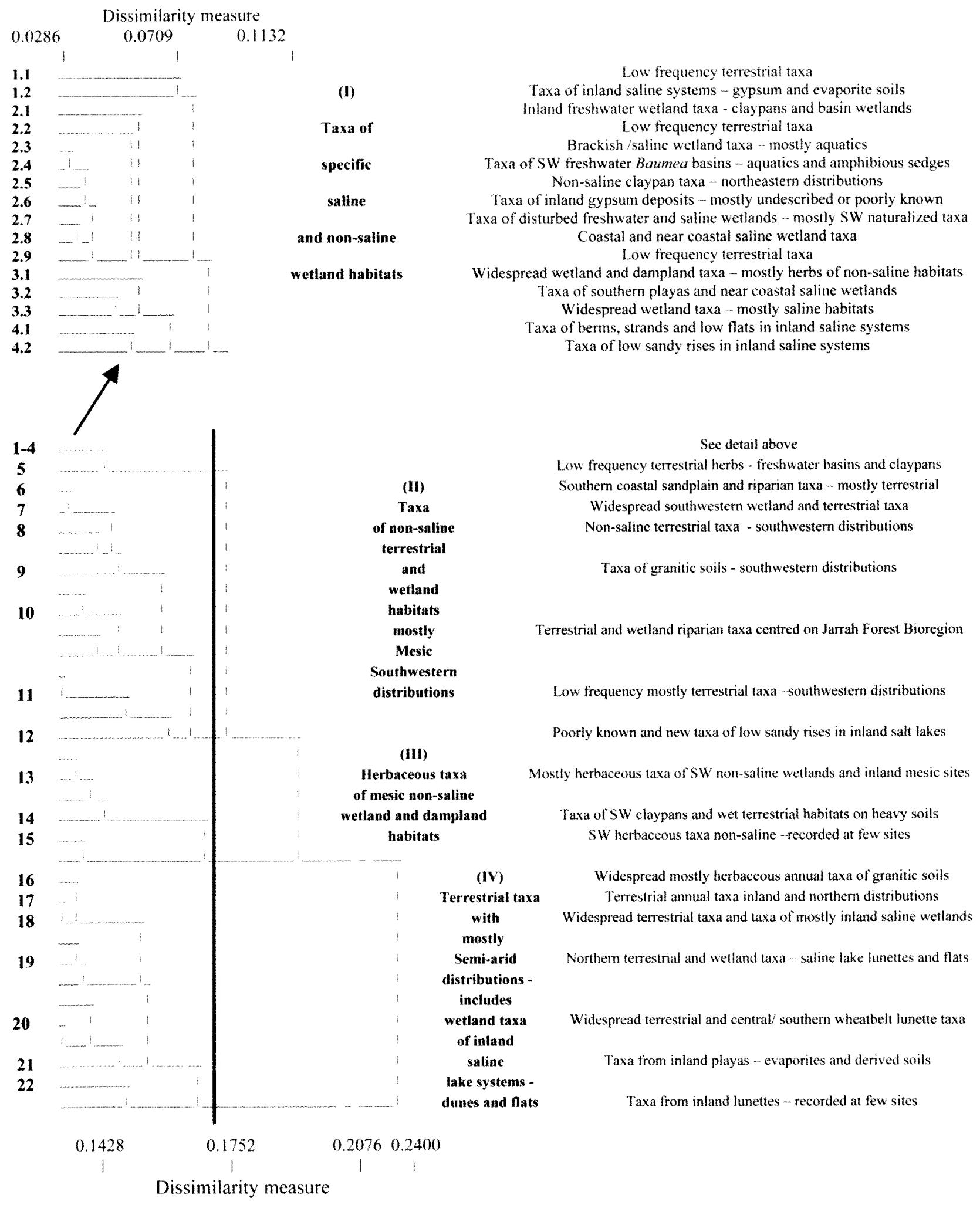

Figure 9 Annotated dendrogram showing the 34-assemblage classification of the 980 species based on their occurrence at 760 quadrats. Broad descriptions are provided at the four (delineated by bold line, roman numerals and comments in bold text) and 34 assemblage level of the classification 

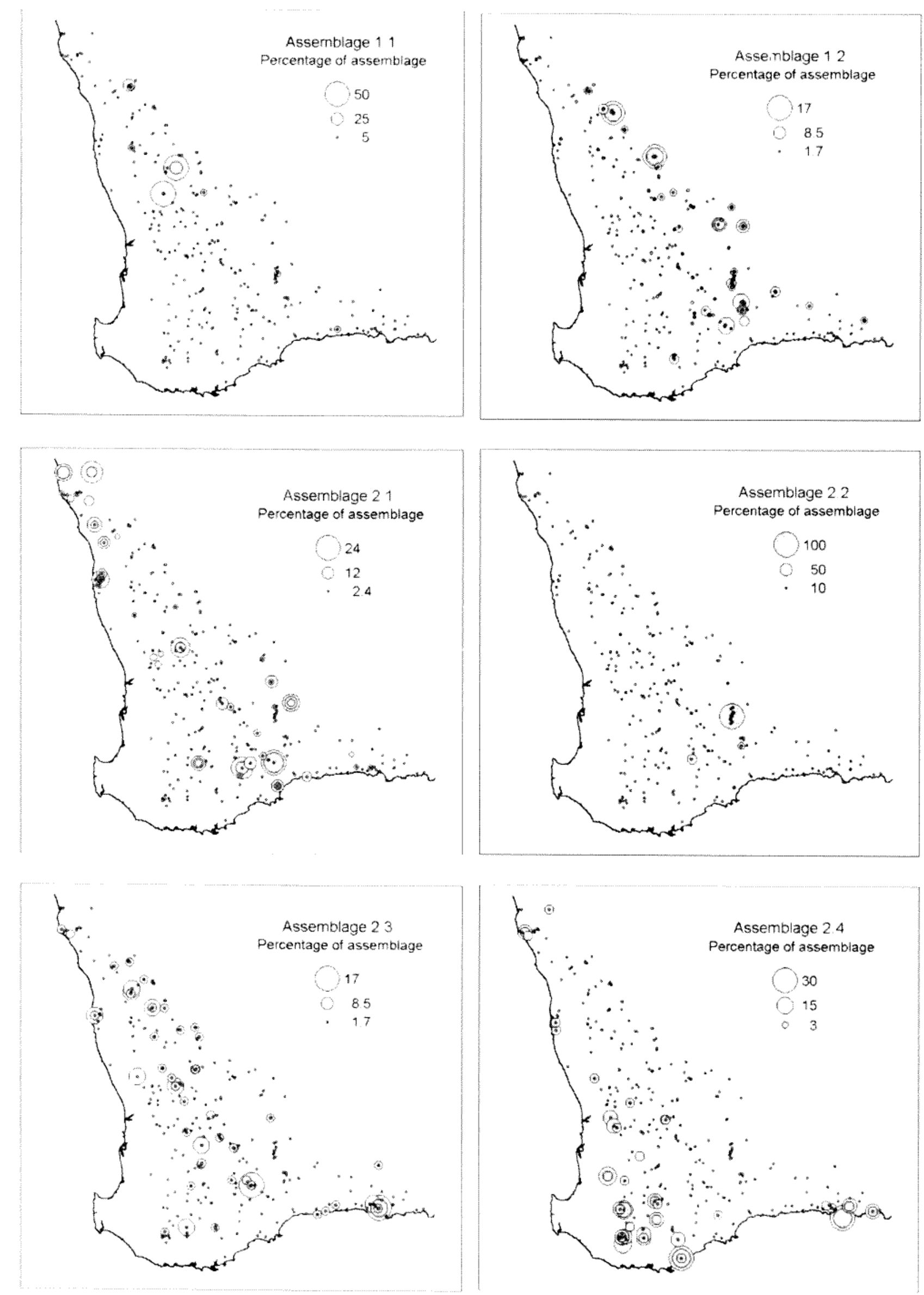

Figure 10 Assemblage richness maps showing the percentage (as dot size) of maximum assemblage richness at all quadrats. 

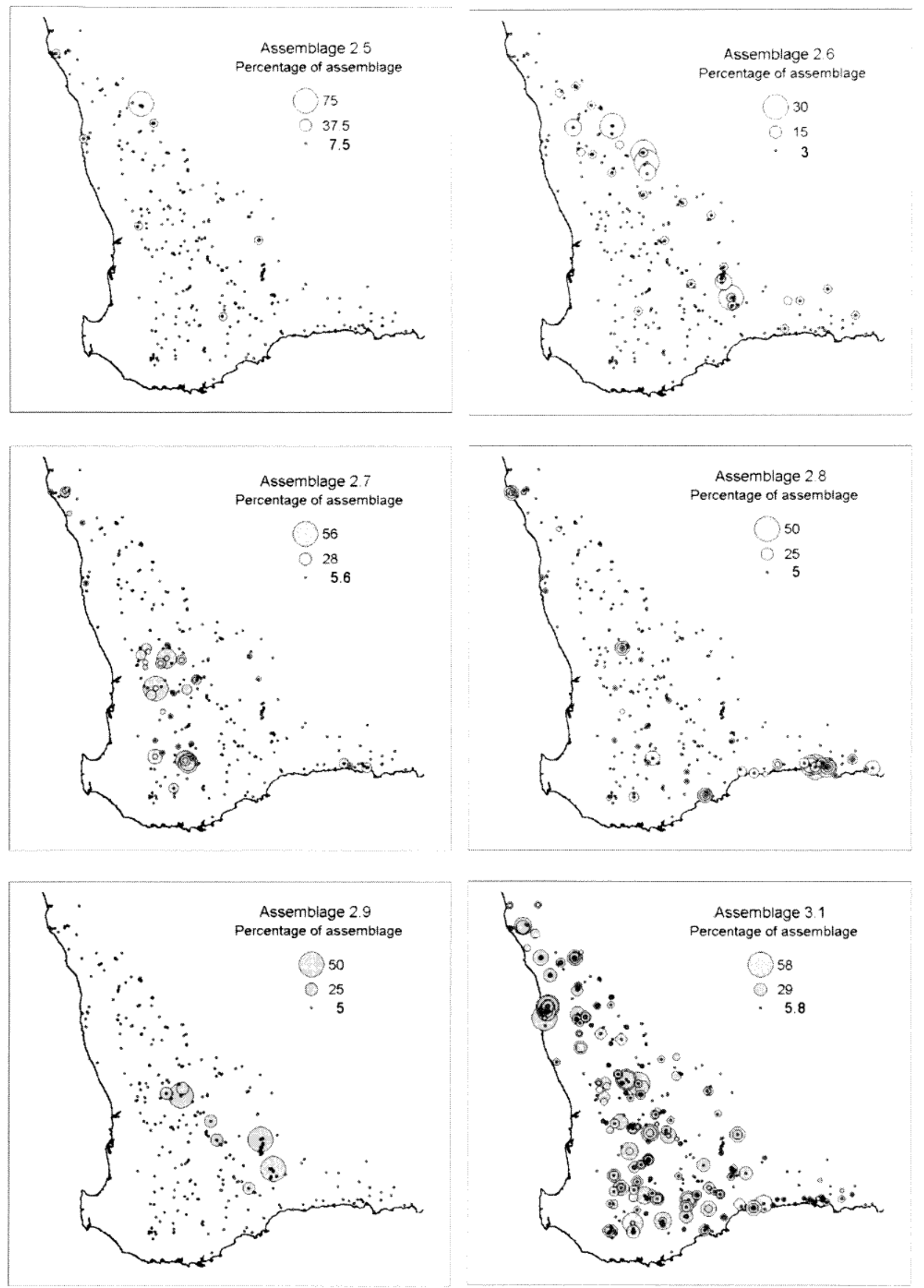

Figure 10 (cont.) 

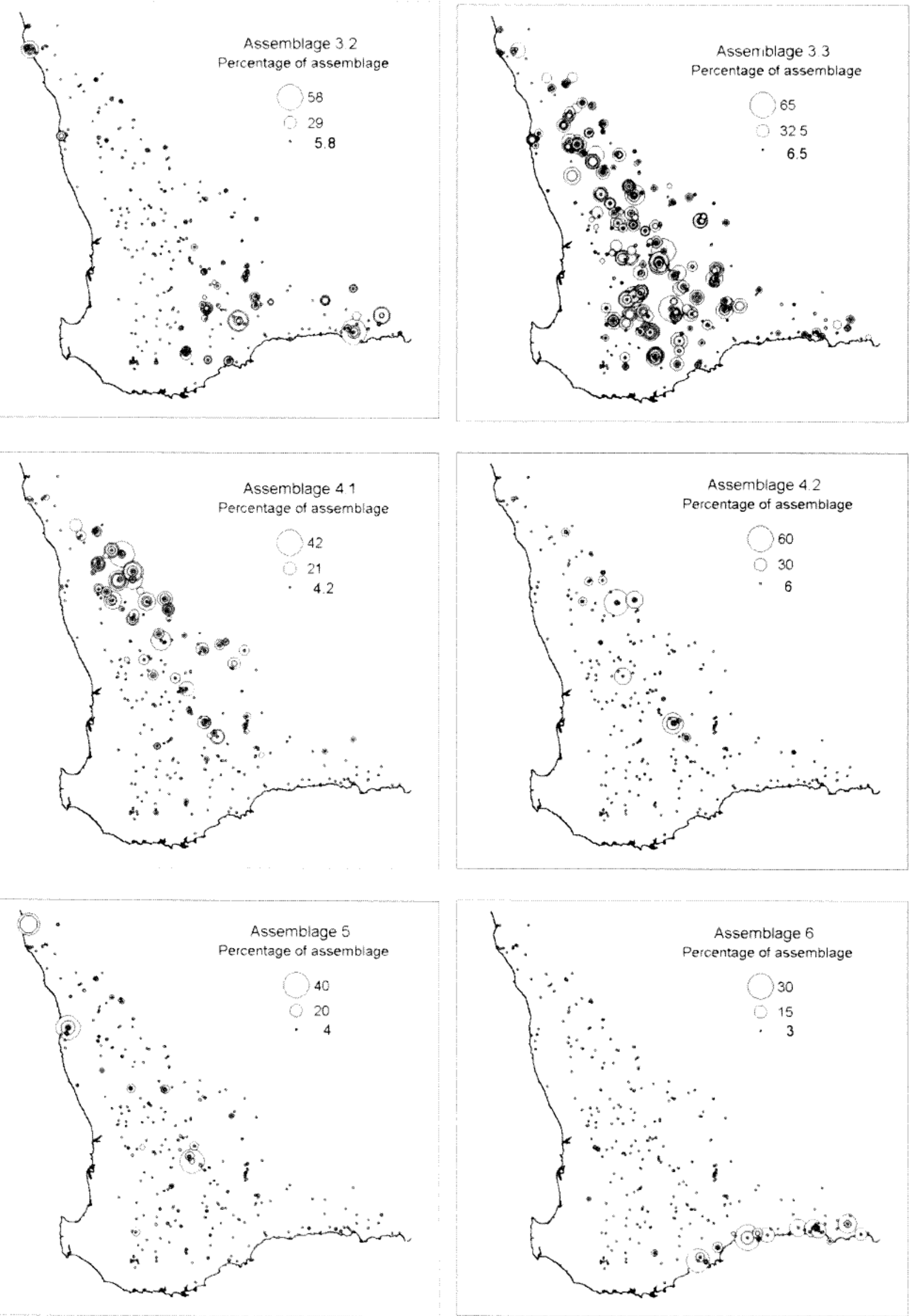

Figure 10 (cont.) 

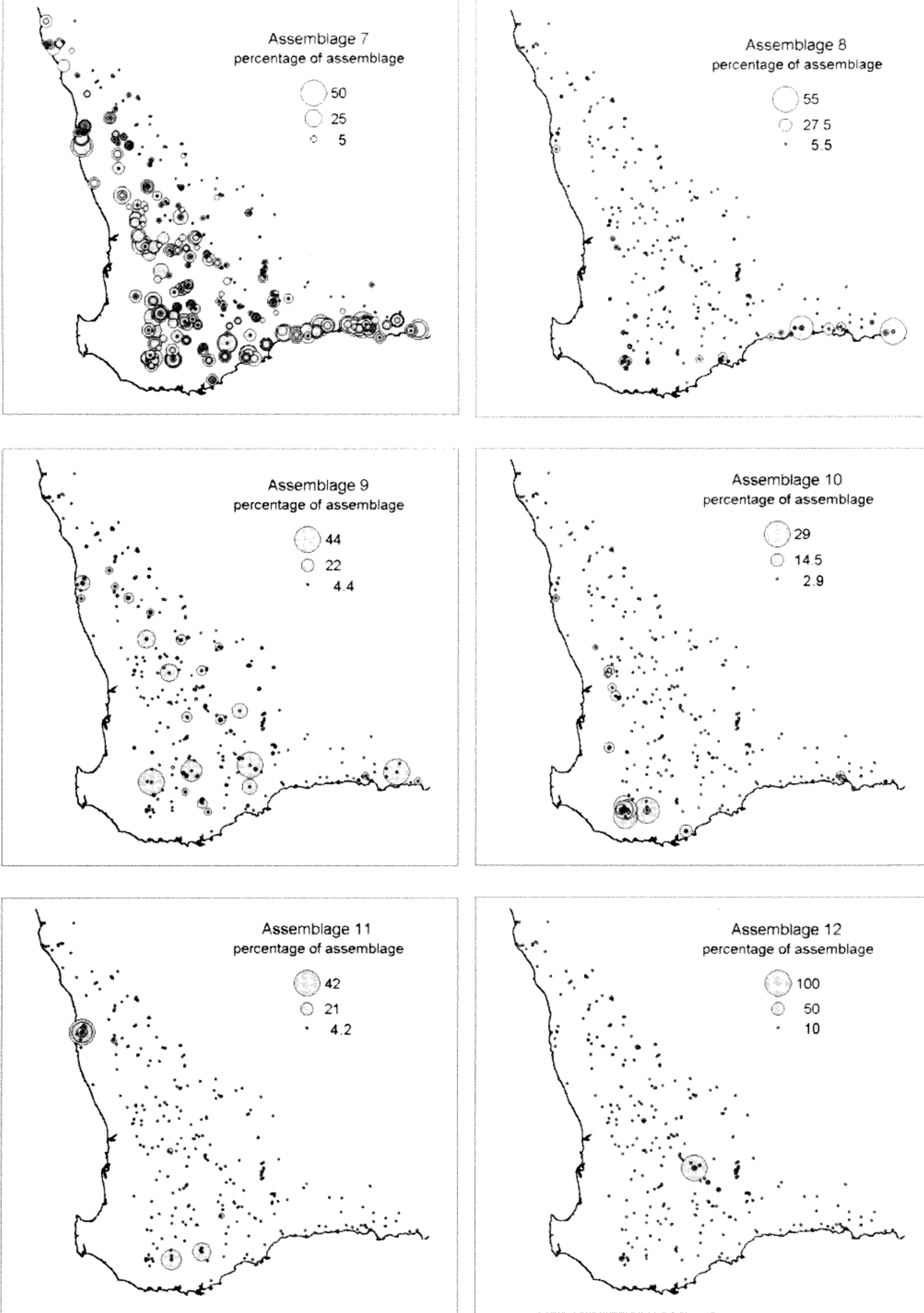

Figure 10 (cont.) 

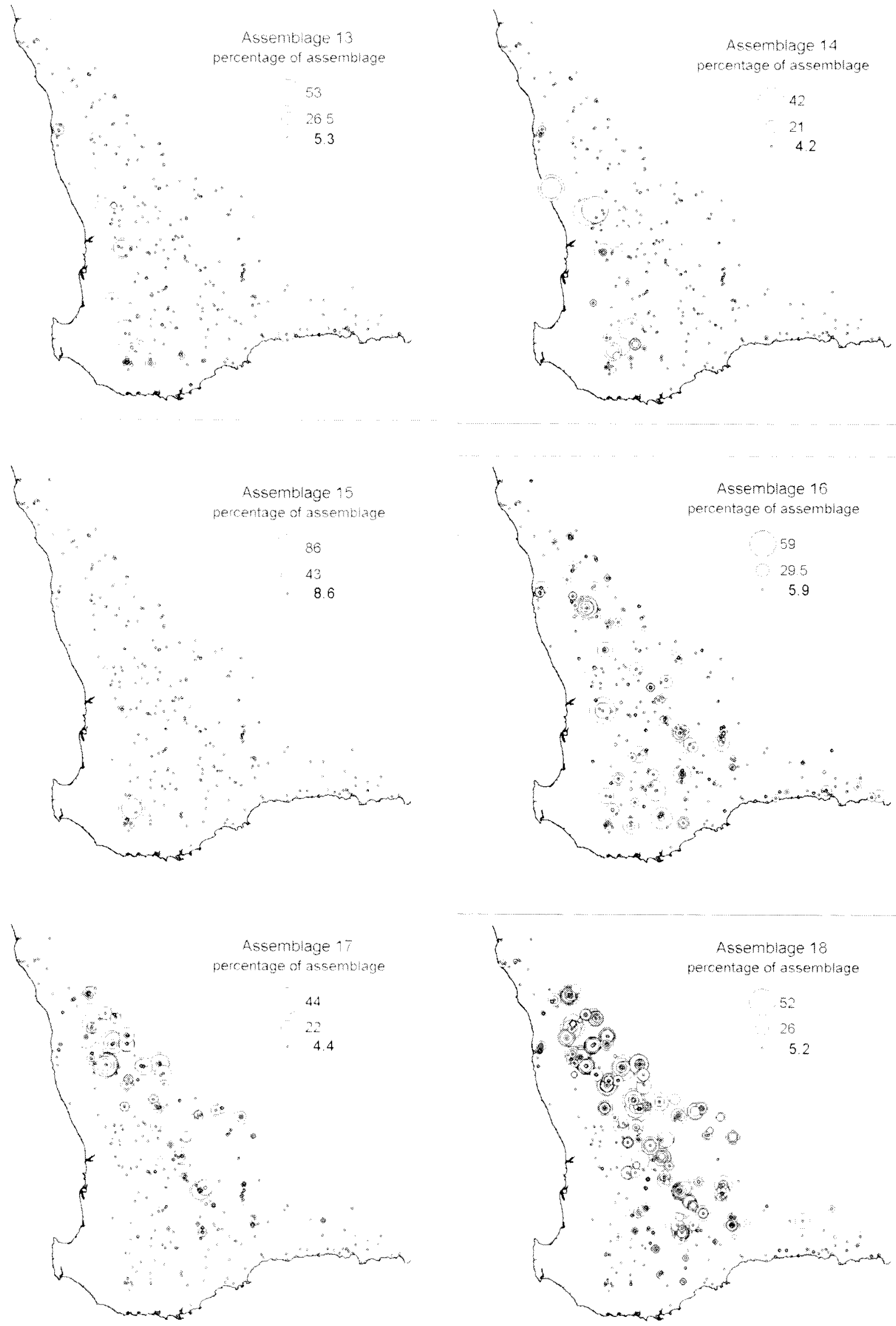

Figure 10 (cont.) 

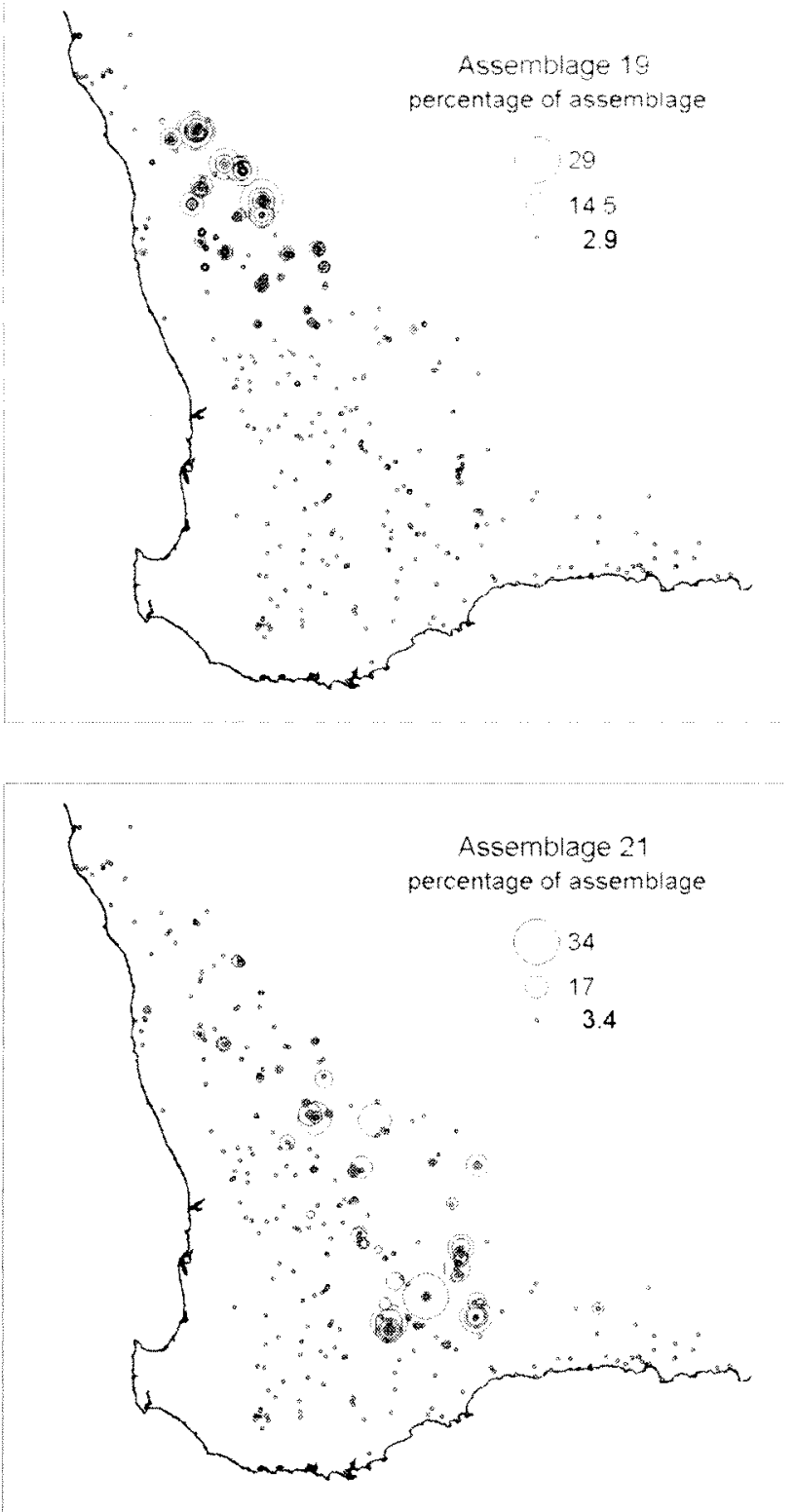

Figure 10 (cont.)

Assemblage 2.5 A small assemblage of taxa from a few quadrats in the north-east of the study area. They occurred in claypans on the arid eastern edge of the study area and are at the southwestern limit of their distributions. They include Myriocephalus gascoynensis and Calandrinia pumila.

Assemblage 2.6 A small group of taxa largely known from gypsum and saline clay substrates on the margins, or on centrally raised beds, of inland saline playas and pans. The assemblage is almost exclusively known from wetlands and occurs in the eastern margin of the Avon Wheatbelt and Mallee bioregions and extends into the adjacent Yalgoo and Coolgardie bioregions. Several members of the assemblage are undescribed and known from few localities. They include Frankenia sp. southern gypsum (Lyons 2914), Halosarcia sp. Lake Moore (M.N. Lyons 2603), Triglochin lyonsii ms (M.N.
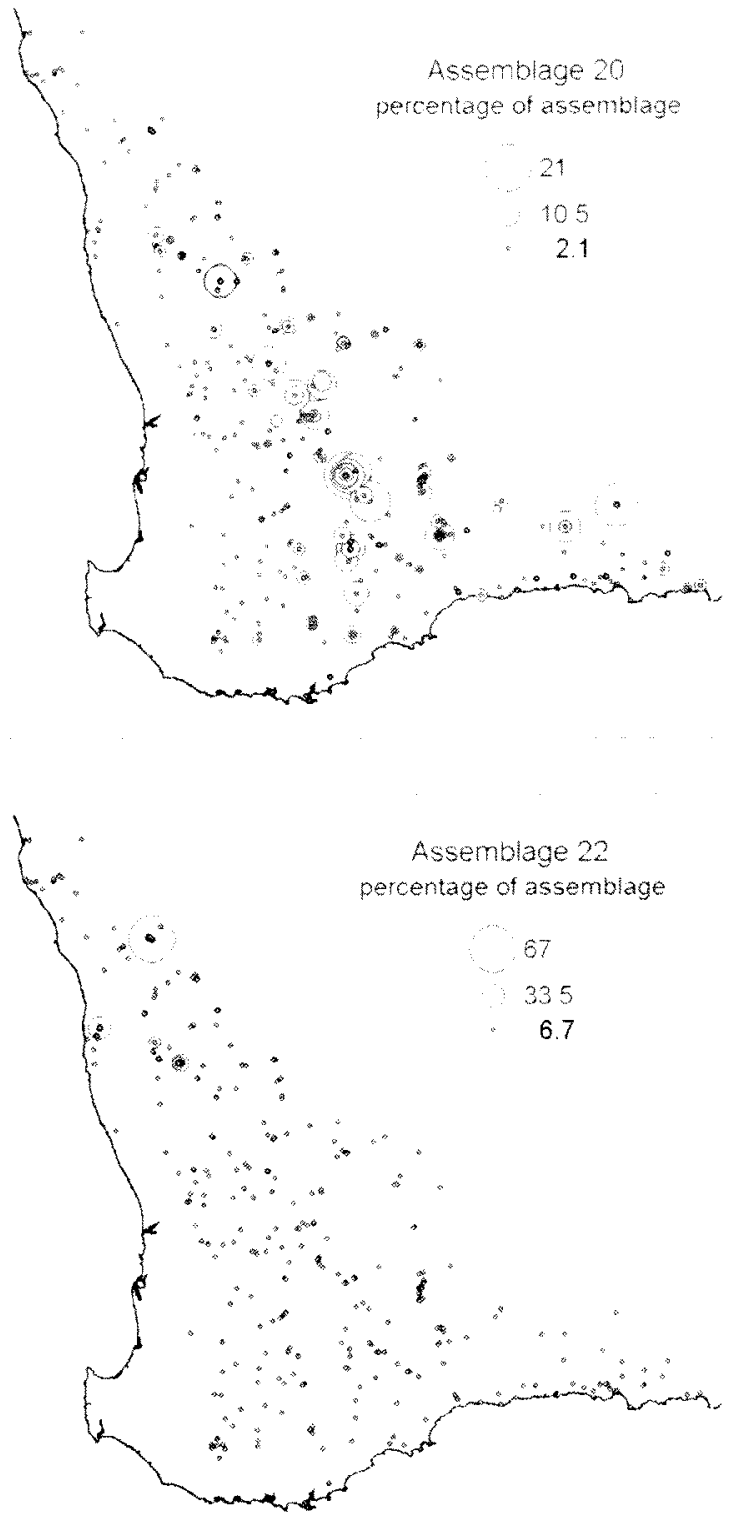

Lyons 2855) and Hydrocotyle hexaptera ms. Other taxa include Chondropyxis halophila and Trichanthodium exile (Figure 10, Table 7, Appendix 4).

Assemblage 2.7 A small group of mostly naturalized species known from disturbed/artificial wetlands, agricultural dam edges and saline/ brackish seeps. The component taxa are exclusively wetland species (Table 7) and include Juncus acutus, Rumex crispus, Symphyotrichum subulatum, Typha domingensis, Puccinellia ciliata and Bolboschoenus caldwellii. The component species are known from the mesic south-west and extend into the western Avon Wheatbelt in suitable habitats. They do not penetrate into the semi-arid arid zone (Appendix 4, Figure 10).

Assemblage 2.8 Taxa of coastal and near coastal, saline wetlands. They occupy beaches, 
Table 7 Number of taxa in each species assemblage that are aquatic, amphibious, occur only in wetland related terrestrial habitats (dunes, riparian zone etc), and those that also occur in non-wetland related terrestrial habitats. Rows labeled with roman numerals are totals for the four-group level species classification.

\begin{tabular}{|c|c|c|c|c|c|c|}
\hline Assemblage & aquatic & amphibious & $\begin{array}{c}\text { Wetland } \\
\text { related }\end{array}$ & $\begin{array}{c}\text { Wetland } \\
\text { total }\end{array}$ & terrestrial & Total \\
\hline 1.1 & - & - & - & - & 8 & 8 \\
\hline 1.2 & - & - & 14 & 14 & 29 & 43 \\
\hline 2.1 & 6 & 11 & 14 & 31 & 11 & 42 \\
\hline 2.2 & - & - & - & - & 7 & 7 \\
\hline 2.3 & 5 & 2 & 6 & 13 & 5 & 18 \\
\hline 2.4 & 4 & 11 & 1 & 16 & 1 & 17 \\
\hline 2.5 & 1 & - & 1 & 2 & 2 & 4 \\
\hline 2.6 & - & - & 8 & 8 & 2 & 10 \\
\hline 2.7 & 2 & 2 & 5 & 9 & - & 9 \\
\hline 2.8 & - & 3 & 4 & 7 & 3 & 10 \\
\hline 2.9 & - & - & - & - & 4 & 4 \\
\hline 3.1 & 1 & 3 & 9 & 13 & 6 & 19 \\
\hline 3.2 & - & - & 16 & 16 & 5 & 21 \\
\hline 3.3 & - & 3 & 13 & 16 & 8 & 24 \\
\hline 4.1 & - & 1 & 21 & 22 & 1 & 23 \\
\hline 4.2 & - & - & 10 & 10 & - & 10 \\
\hline 5 & 1 & 2 & 3 & 6 & 17 & 23 \\
\hline (I) & 20 & 37 & 125 & 183 & 109 & 292 \\
\hline 6 & - & 2 & 5 & 7 & 33 & 40 \\
\hline 7 & - & 7 & 6 & 13 & 21 & 34 \\
\hline 8 & - & - & - & - & 33 & 33 \\
\hline 9 & - & - & - & - & 16 & 16 \\
\hline 10 & - & 10 & 10 & 20 & 60 & 80 \\
\hline 11 & - & - & 3 & 3 & 21 & 24 \\
\hline 12 & - & - & 6 & 6 & - & 6 \\
\hline (II) & - & 19 & 30 & 49 & 184 & 233 \\
\hline 13 & - & - & 6 & 6 & 40 & 46 \\
\hline 14 & 12 & 10 & 13 & 35 & 15 & 50 \\
\hline 15 & 2 & 3 & 7 & 12 & 16 & 28 \\
\hline (III) & 14 & 13 & 26 & 53 & 71 & 124 \\
\hline 16 & - & 1 & - & 1 & 23 & 24 \\
\hline 17 & - & - & 5 & 5 & 45 & 50 \\
\hline 18 & - & - & 15 & 15 & 53 & 68 \\
\hline 19 & - & - & 20 & 20 & 44 & 64 \\
\hline 20 & - & - & 7 & 7 & 70 & 77 \\
\hline 21 & - & - & - & - & 36 & 36 \\
\hline 22 & - & - & - & - & 12 & 12 \\
\hline (IV) & - & 1 & 47 & 48 & 283 & 331 \\
\hline Total & 34 & 71 & 228 & 333 & 647 & 980 \\
\hline
\end{tabular}

estuarine margins, coastal swamps, near coastal playas and more inland saline seeps and springs. Most are restricted to coastal parts of the Warren, Swan Coastal Plain, Geraldton Sandplains and Esperance Sandplains bioregions. They include Sporobolus virginicus, Wilsonia backhousei, Suaeda australis, Vellea exigua and Triglochin striatum. A few taxa extend into the Jarrah Forest and western Avon Wheatbelt bioregions (e.g. Juncus kraussii subsp. australiensis, Chenopodium glaucum).
Seven of the ten taxa only occur within wetlands (Table 7).

Assemblage 2.9 A small artifactual group of terrestrial taxa recorded from few quadrats. The species clustering was driven by two quadrats sampling the edges of inland dams.

Assemblage 3.1 A group dominated by ubiquitous wetland taxa distributed in various wetland habitats throughout the south-west. A few taxa extend into the Coolgardie bioregion (e.g. 
Melaleuca strobophylla, Agrostis avenacea). The component taxa occur in a wide variety of mostly non-saline wetland habitats. They include freshwater basin wetlands and margins, granite rock pools and herb swards, winter-wet terrestrial sites, disturbed freshwater and brackish wetlands and some small scale habitats (seasonally leached nonsaline sands) on the margins of salt lakes and playas (see quadrat group 8.2). The assemblage contains several introduced taxa including Pseudognaphalium luteoalbum, Cotula coronopifolia, Lythrum hyssopifolia, Juncus bufonius and Polypogon monspeliensis. Centrolepis polygyna and Isolepis cernua are common native members (Appendix 4, Figure 10).

Assemblage 3.2 Taxa of coastal and near coastal salt lakes and southern inland saline playas. Most taxa (16 of 21) only occur in wetland habitats but some such as Austrostipa pycnostachya occur in some non-wetland-associated woodlands and shrublands. The assemblage includes taxa that have southern distributions within the Esperance Sandplains, southern Avon Wheatbelt and Mallee bioregions (Wilsonia humilis, Hagiela tatei, Frankenia tetrapetala, Lawrencia glomerata, Tegicornia uniflora and Austrostipa juncifolia). Others such as Samolus repens, Isotoma scapigera and Angianthus preissianus are more widespread coastal and near coastal species. (Appendix 4, Figure 9). Within wetlands the taxa occupy berms and elevated flats, often of evaporite derived substrates. The assemblage is exclusively comprised of native taxa.

Assemblage 3.3 Ubiquitous taxa of primary and secondary saline/brackish and disturbed freshwater wetland edges. Widespread taxa include Halosarcia indica subsp. bidens, Sarcocornia quinqueflora, Cotula bipinnata, Triglochin mucronata, Apium annum and Parapholis incurva (Appendix 4, Figure 10). The majority of the component taxa have coastal and wheatbelt distributions, which largely exclude the Jarrah Forest bioregion due to lack of suitable habitat (e.g. Sarcocornia quinqueflora, Halosarcia pergranulata and Casuarina obesa). A subset of taxa such as Melaleuca halmaturorum and Halosarcia doleiformis do not occur on the coast and have distributions that extend into the adjacent arid zone. Two-thirds of the assemblage's taxa are restricted to wetland habitats and several are very widespread wetland weeds, particularly in habitats with some saline influence (e.g. Spergularia rubra and Cotula bipinnata). Taxa not restricted to wetlands include Hordeum glaucum, H. leporinum and Lolium spp.

Assemblage 4.1 Taxa occurring on sandy berms, strand lines and low sandy flats of inland primarily saline playas and braided saline drainage-lines. The assemblage does not contain any introduced taxa and its component taxa have distributions that include the wheatbelt and extend into the adjacent arid zone within paleo-drainage systems. The assemblage includes seven species of Halosarcia and most have distributions that follow this pattern (Halosarcia undulata, $H$. halocnemoides and $H$. pruinosa). A subset of taxa are more strongly distributed in the northern Avon Wheatbelt, adjacent Yalgoo and some further inland (Frankenia conferta, Gunniopsis septifraga, Halosarcia peltata and H. pruinosa). A further group of taxa have relatively narrow geographic ranges in the northern Avon Wheatbelt and adjacent Yalgoo bioregions (Gnephosis setifera, Gnephosis cassiniana and Neosciadium glochidiatum) (Appendix 4, Figure 10). Some northern taxa are associated with gypsum soils on the margins of salt lakes (Frankenia conferta and Neosciadium glochidiatum).

Assemblage 4.2 A group of taxa including Roycea pycnophylloides, Sarcocornia globosa, Halosarcia sp. Central Wheatbelt (M.N. Lyons \& S.D. Lyons 2760), Frankenia bracteata and Drosera zigzagia. They occur at the edges of channels in braided primary saline drainage lines, slightly elevated sandy rises and margins of primary saline playas and small pans. Most taxa are known from relatively few localities and are distributed within the Avon Wheatbelt and Mallee bioregions (Appendix 4, Figure 10). All are restricted to wetland habitats (Table 7). Prior to the current survey their distributions and habitat preferences were poorly known.

Assemblage $5 \mathrm{~A}$ heterogeneous group of taxa that includes a group of inland freshwater wetland species (e.g. Damasonium minus and Lythrum wilsonii) and a group of introduced taxa, some of which are restricted to wetlands (Phalaris paradoxa, P. minor and Myosurus minima var. australis). The majority of the assemblage's taxa however are not restricted to wetlands (ca. $73 \%$ ) and some are widespread in temperate and semi-arid terrestrial habitats (e.g. Emex australis and Centaurea melitensis) (Table 7, Appendix 4). The majority of the taxa occur in terrestrial and wetland habitats that are non-saline with fine textured soils.

Assemblages of non-saline terrestrial and wetland habitats in the mesic south-zvest

Assemblage 6 An assemblage dominated by taxa with distributions strongly centred within the Esperance Sandplains bioregion occurring on nonsaline sandy soils (Hopkinsia adscendens, Corynotheca micrantha subsp. panda, Eutaxia cuneata, Hypolaena humilis, Acacia subcaerulea, Conostylis seorsiflora subsp. seorsiflora and Melaleuca incana subsp. tenella). Other taxa have wider distributions within the remainder of the higher rainfall areas of the southwest. They variously extend into the Warren, Jarrah Forest and Swan Coastal Plain, southern Avon Wheatbelt and southern Mallee bioregions (Anarthria laevis, Hakea nitida, Darwinia vestita and Hypolaena exsulca) (Appendix 4, Figures 4 and 10). While few taxa are restricted to wetlands $(17.5 \%)$, several tend to extend to other seasonally wet 
habitats (Hypolaena exsulca, H. humilis and Anarthria laevis). Within wetlands the component taxa occupy dunes and beaches typically beyond the zone of inundation.

Assemblage $7 \mathrm{~A}$ widespread group of taxa that occur at a range of wetland types in the higher rainfall southwestern and coastal parts of the study area. Over a third (38\%) of the component taxa are restricted to wetland habitats and occupy wooded swamps, sedge swamps, riverbanks (fresh and saline) and near coastal lake margins. Examples include Lepidosperma longitudinale, Isolepis nodosa, Villarsia parnassifolia, Melaleuca cuticularis, Eucalyptus occidentalis, Melaleuca rhaphiophylla, Baumea inncea. Juncus pallidus and Gahmia trifida (Appendix 4, Figure 10). The remainder of the assemblage occurs widely in terrestrial habitats and includes a group of naturalized taxa that are ubiquitous in mesic and temperate parts of southwestern Australia (e.g. Anagallis arvensis, Briza maxima, B. minor and Ehrharta longiflora). The assemblage occurs at a variety of wetland positions.

Assemblage 8 A disparate assemblage of terrestrial taxa that occupy non-saline habitats. The assemblage was largely associated with sands but contained a small subset found on clavs (Acacia glaucoptera, Hakea laurina). They are distributed in the higher rainfall part of the study area. The assemblage includes taxa with distributions in the Esperance Sandplains, southern Avon Wheatbelt and adjacent Mallee bioregions, such as Hakea laurina, Acacia glaucoptera and Lomandra micrantha subsp. teretifolia. Others have coastal distributions associated with consolidated dunes (Acacia rostellifera, Leucopogon parviflorus, Templetonia retusa and Spyridium globulosum) or more extensive southwestern distributions (Hakea lissocarpha, Lagenifera huegelii, Austrostipa mollis, Microlaena stipoides and Chamaescilla corymbosa) (Appendix 4 , Figure 10).

Assemblage 9 A small group of terrestrial taxa with southwestern distributions that occupy nonsaline sites. The assemblage includes taxa typical of granite derived soils (granite aprons and orange sands) including Allocasuarina huegeliana, Neurachu alopecuroidea, Centrolepis drummondiana and Stypandraglauca

Assemblage 10 A large group of taxa with southwestern distributions. Taxa include trees, shrubs, sedges and perennial herbs that occur in the riparian zones of fresh lakes, rivers and sedge swamps. Most taxa however are not restricted to wetlands (75\%, e.g. Acacia pulchella, Macrozamia riedlei, Leucopogon australis and Xanthosia huegelii), although several extend only to seasonally damp habitats particularly in the high rainfall south west (e.g. Lepidosperma striatum and Drosera pulchella). Wetland species include Melaleuca preissiana, Viminaria juncea, Banksia littoralis, Agonis juniperina,
Patersonia sp. Swamp form (N. Gibson and M. Lyons 544) and Boronia spathulata. Annual herbs are poorly represented in the assemblage. The distribution of the component taxa of the assemblage is centred in the Jarrah Forest, Warren and Swan Coastal Plain bioregions (Appendix 4, Figure 10).

Assemblage $11 \mathrm{~A}$ small group of predominantly non-wetland restricted taxa with southwestern affinities recorded at very few non-saline quadrats. They occurred in similar habitats to the members of assemblage 10. The assemblage clustering is driven by species co-occurrences at three quadrats (Appendix 4, Figure 10). The assemblage was very poorly sampled.

Assemblage 12 A small group of wetland taxa known from the sandy margins of saline playas and braided drainage lines in the Mallee, Esperance Sandplains bioregions and adjacent southern extremities of the Avon Wheatbelt and Jarrah Forest bioregions. Prior to the current survey the distributions and habitat preferences of the taxa were very poorly known. Members of the assemblage are still known from only few populations and include undescribed taxa such as Tribonanthes minuta ms (M.N. Lyons 2730), Hypoxts salina ms (M.N. Lyons 2735) and Calandrinia sp. Hyden (R.J. Cranfield 11298) (Appendix 4, Figure 10).

Herbaceous taxa of mesic - non-saline wetland habitats

Assemblage 13 A large assemblage of mostly herbaceous taxa centred on the Jarrah Forest, Warren and Swan Coastal Plain bioregions. The taxa occupy non-saline seasonally wet habitats, often with fine textured soils, but most extend into non-wetland habitats, particularly in the high rainfall areas of the south-west (Table 7, Appendix 4). They occurred on the margins of freshwater lakes, sedge-swamps, claypans and wet clay flats. While most occur in the high rainfall zone some taxa have distributions that extend into the Avon Wheatbelt, although there they are restricted to the margins of freshwater wetlands and/or granite outcrops (e.g. Centrolepis aristata, Aphelia brizula and Schoenus odontocarpus) (Appendix 4, Figure 10).

Assemblage 14 An assemblage dominated by freshwater wetland taxa. Wetland taxa represent $70 \%$ of the assemblage and $44 \%$ are aquatic or amphibious plants (Table 7). The taxa occur in freshwater claypans and their margins (e.g. Amphibromus nervosus, Rhodanthe pyrethrum, Chorizandra enodis, Eleocharis keigheryi, Microtis orbiculare and the aquatics Schoenus natans and Hydrocotyle lemoides) but also in seasonally damp terrestrial habitats with fine textured soils (e.g. Wurmbea monantha, Hakea varia and Centrolepis alepyroides). The assemblage is centred on the Swan Coastal Plain, Jarrah Forest, southern Geraldton Sandplains and the western margin of the Avon Wheatbelt bioregions (Figure 10). 
Assemblage 15 A group of mostly herbaceous taxa that occupy similar freshwater habitats to assemblage 13 and 14 , including sedge swamps, winter-wet flats and claypans. Approximately $43 \%$ of taxa only occur in wetlands, while the remainder occupy damp/wet terrestrial habitats particularly in the high rainfall areas of the south-west. The assemblage is strongly centred within the Warren, Swan Coastal Plain and southern Jarrah Forest bioregions. Most component taxa are relatively widespread within the high rainfall zone although a few, such as Tribonanthes sp. Lake Muir (G.J. Keighery and N. Gibson 2134), are only known from the Lake Muir group of wetlands (Appendix 4, Figure 10). A few taxa within the assemblage occur in the western margin of the Avon Wheatbelt bioregion on granite rock outcrops (Hydrocotyle callicarpa, Aphelia nutans). The assemblage's core distribution was beyond the boundaries of the current study.

Widespread terrestrial taxa (semi-arid and arid distributions) and taxa of inland salt lake systems

Assemblage 16 A group dominated by widespread herbs, with distributions centred on the Avon Wheatbelt bioregion. Many taxa have broader distributions that include the Jarrah Forest, Swan Coastal Plain, Mallee bioregions, with some penetrating semi-arid Coolgardie and Yalgoo bioregions. The component taxa are generally absent from the high rainfall areas of the south-west corner. Taxa include Acacia acuminata, Eucalyptus loxophleba subsp. loxophleba, Gonocarpus nodulosus, Quinetia urvillei, Drosera glanduligera, Millotia tenuifolia, Dianella revoluta, Podolepis lessonii and Daucus glochidiatus (Figure 10, Appendix 4). None of the taxa are restricted to wetlands and most are widespread on more fertile granitic soils, orange sandy loams and finer textured valley floor soils that are non-saline.

Assemblage 17 An assemblage of taxa typical of semi-arid Acacia, Eremophila and Melaleuca dominated shrublands and eucalypt woodlands. The group is a combination of widespread lower rainfall non-wetland annuals and taxa specific to shrublands on lunettes and flats within salt lake systems (e.g. Lawrencella rosea and Actinobole uliginosum cf. Sarcozona praecox and Podotheca pritzelii). The assemblage is centred within the central and northeastern parts of the study area (Avon Wheatbelt 2 bioregion) yet many taxa extend into adjacent bioregions particularly to the north and east (Yalgoo, Coolgardie, Carnarvon Basin and Murchison). The taxa show preference for more fertile granite derived soils.

Assemblage 18 A group of taxa dominated by annual herbs that have distributions centred within the Avon Wheatbelt and Mallee and extending into the Yalgoo and Coolgardie bioregions. Taxa within the assemblage display a variety of habitat preferences but most are associated with saline and semi-saline habitats. Those taxa within the assemblage restricted to wetlands (ca. $22 \%$ ) occur in habitats on the margins of naturally saline playas and brained saline drainage systems. They include Didymanthus roei, Gnephosis angianthoides, Stenopetalum salicola, Centrolepis humillima and Atriplex hymenotheca. Many of the remaining taxa, though not restricted to wetlands tend to occur in habitats with some saline influence e.g. Lycium australe, Threlkeldia diffusa (largely coastal), Pogonolepis stricta, Rhagodia drummondii, Mesembryanthemum nodiflorum and Atriplex paludosa. The assemblage includes a small group of ubiquitous introduced taxa such as Hypochaeris glabra, Arctotheca calendula and Vulpia spp.

Assemblage 19 A group of species that have semi-arid and arid zone distributions that include the northern Avon Wheatbelt, Geraldton Sandplains, Yalgoo bioregions and extend into the Gascoyne, Murchison, Coolgardie and southern Carnarvon Basin. Compared to assemblage 16 and 17 , the component taxa are more strongly restricted to primary salt lake habitats, e.g. Gunniopsis quadrifida, Eremophila miniata, Maireana amoena and M. atkinsoniana (ca. $31 \%$ of taxa are restricted to wetlands, Table 7) or non-wetland habitats of valley floors. Some taxa such as Epitriche demissum, Dithyrostegia amplexicaulis and Brachyscome halophila only occur on the margins of saline playas and have narrow geographic ranges centred in the northern Avon Wheatbelt and Yalgoo bioregions. A number of taxa occur in various habitats within the arid zone and are at the southwestern limit of their distributions in the north-east of the study area, e.g. Acacia ramulosa subsp. ramulosa, Solanum orbiculare, Calocephalus francisii and Brunonia australis (Appendix 4, Figure 10).

Assemblage 20 This assemblage is the central and southern wheatbelt analogue of assemblages 17 and 19. The group is centred within the Avon Wheatbelt and Mallee bioregions and extends to the adjacent western edge of the Coolgardie. The assemblage is dominated by taxa not restricted to wetlands (ca. $91 \%$, particularly widespread annual herbs, although the distributions of many shrub and tree taxa only extend to adjacent valley floor communities (e.g. Carpobrotus modestus and Melaleuca brophyi). Within wetland habitats the assemblage occurs on elevated flats and dunes in primary salt lake systems. Taxa restricted to wetlands include Eucalyptus sargentii, E. salicola, Darwinia halophila, Bossiaea halophila and Lomandra micrantha subsp. teretifolia "robust form" (A.S. George 14295) (Appendix 4, Figure 10).

Assemblage 21 Taxa occurring on finer textured soils of valley floors and broad aeolian plains associated with primary salt lake systems. No 
members of the assemblage are restricted to wetland habitats, although several are strongly associated with primarily saline wetlands, e.g. Acacia inceana subsp. conformis, Millotia major and Eucalyptus kondininensis. The assemblage includes a number of widespread naturalized taxa including Moraca setifolia, Erodium cicutarium and Trifolium aroense var. arvense. The assemblage is centred within the Southern Avon Wheatbelt and Mallee bioregions, but extends to include the Coolgardie and northern Avon Wheatbelt bioregions. It is generally absent from the higher rainfall areas of the south-west (Appendix 4, Figure 10).

Assemblage 22 A small disparate group of terresterial taxa centred in the Avon Wheatbelt, Geraldton Sandplains and Yalgoo bioregions. Some taxa extend into the adjacent arid zone north-east of the study area. With the exception of Swainsona perlonga, they are associated with non-saline soils such as sand dunes and sand sheets (e.g. Triodia longipalea) or granite derived soils (e.g. Levenhookia dubia and Ophioglossum gramineum; Appendix 4, Figure 10).

Most assemblages were geographically well defined, but a few, such as assemblages 2.1 and 5 , were patchily distributed across the study area in the small number of suitable habitats sampled (e.g. taxa of fresh swamps and claypans) (Figure 10). Several assemblages occurred at the margins of the study area and represent groups of taxa whose core ranges lie outside the study area (e.g. assemblages $7,10,13,14$ and elements of assemblages 17 and 19). For some assemblages of naturally saline areas, it is difficult to assess the extent to which they extend into adjacent areas of the semi-arid zone (e.g. assemblages 18 and 21).

\section{Species assemblages and environmental models}

Assessments of each GLM model are described below. Models were assessed in terms of their consistency with knowledge of the distribution and habitat preferences of the component taxa.

Assemblage 1.1 was considered an artifact and modeling iterations did not converge.

Assemblage 1.2 showed a strong unimodal relationship between composition and species richness and an acceptable fit to the Poisson error model. The model described $47.1 \%$ of the scaled deviance of the null model. The significant terms in the GLM model (Table 8) were consistent with the known distribution and habitat preferences of the taxa in the assemblage. High richness was associated with inland salt lakes and associated valley floors (low winter rainfall). The assemblage was richest on fine textured calcareous and gypseous soils and this was reflected by the inclusion of $\mathrm{pH}$ (moderate to high $\mathrm{pH}$ ) and phosphous (moderate) in the model. The assemblage is not associated with the parts of wetlands subject to inundation (moderate to high ELCODE) (Tables 8 and 9).

Assemblage 2.1 showed a strong unimodal relationship between composition and species richness and a moderate fit to the Poisson model. The model accounted for $48.8 \%$ of the scaled deviance of the null model. Model terms were consistent with the assemblages' occurrence in the basins and margins (low ELCODE) of freshwater swamps (low EC). The assemblage is associated with wetlands largely outside the high rainfall mesic south-west corner and this was reflected in the inclusion of the model term annual precipitation (low) (Tables 8 and 9).

Assemblage 2.2 was considered an artifact and modeling iterations did not converge.

Assemblage 2.3 The occurrences of this assemblage were very species poor (maximum of 3 taxa/quadrat) and the data did not conform to the Poison model assumed by the GLM procedure. The one-dimensional ordination plotted against species richness was however unimodal. The GLM model explained only $33.6 \%$ of the null model scaled deviance (Table 9). Despite this, model terms broadly reflected the known habitat preferences of the assemblage's taxa. Maximum species richness occurred in the basins and margins of saline wetlands (low ELCODE) and moderate to high EC (Table 8). The assemblage was absent from acidic wetlands (fresh and saline) and the GLM model reflected this in the inclusion of the model term $\mathrm{pH}$ (moderate to high $\mathrm{pH}$ ).

Assemblage 2.4 showed a poor relationship between composition and species richness. This is partly related to the species poor nature of occurrences of this assemblage. At any quadrat, even in habitats deemed optimal, only a few members of the assemblage were found to be present. The model explained $66.3 \%$ of the scaled deviance of the null model. The terms of the model are consistent with the broader distribution of the assemblage's taxa. They occurred in the basins of wetlands (low ELCODE), predominantly in the south-west but extending $\mathrm{N}$ and $\mathrm{E}$ close to the coast (low maximum temperature of the warmest period). Richest quadrats have low $\mathrm{pH}$ associated with inundated substrates high in organic matter (low pH, Tables 8 and 9).

Assemblage 2.5 contained four taxa sampled at the extreme edge of their range in the north-east of the study area. The assemblage was poorly sampled and modeling iterations did not converge.

Assemblage 2.6 showed a strong relationship between composition and species richness and richness was regarded as a good surrogate for composition. The Poisson fit however was poor. This assemblage is a small group of taxa that occur on gypseous substrates in species poor stands (maximum occurrence 3 taxa) within inland salt 
Table 8 Generalized linear models (GLM) of the relationship between soil attributes, elevation code (ELCODE), geographic location, climatic attributes and assemblage richness. See Table 8 for explanation of variables. Parameter 1 is the intercept. Reported model terms are significant at $P<0.05$ or less (except for intercept for model 3.1). Squared terms are denoted by superscript 2. The number of quadrats excluded to achieve stable models is shown.

\begin{tabular}{|c|c|c|c|c|c|c|c|}
\hline & parameter & estimate & s.e. & & parameter & estimate & s.e. \\
\hline \multirow[t]{2}{*}{1.1} & \multirow{2}{*}{\multicolumn{3}{|c|}{ Model does not converge }} & 2.6 & 1 & 14.89 & 5.805 \\
\hline & & & & & $\mathrm{MG}$ & 0.3758 & 0.05851 \\
\hline \multirow[t]{13}{*}{1.2} & 1 & -15.19 & 4.561 & & GYP & 0.03357 & 0.003721 \\
\hline & $\mathrm{PH}$ & 3.323 & 1.208 & & LAT & -0.4812 & 0.1584 \\
\hline & $\mathrm{PH}^{2}$ & -0.1866 & 0.08094 & & PSEAS & -0.05257 & 0.01886 \\
\hline & PCLDQ & -0.03710 & 0.004691 & & OC & -1.715 & 0.5129 \\
\hline & ELCODE & 2.634 & 0.7026 & & \multicolumn{3}{|c|}{ Scaled deviance $=147.65(739)$} \\
\hline & ELCODE $^{2}$ & -0.3859 & 0.1224 & & \multirow{2}{*}{\multicolumn{3}{|c|}{ Null model Scaled deviance $=311.41$}} \\
\hline & $\mathrm{P}$ & 0.01813 & 0.003369 & & & & \\
\hline & $\mathrm{P}^{2}$ & $-2.349 \mathrm{e}-05$ & $8.679 \mathrm{e}-06$ & 2.7 & 1 & -92.51 & 20.87 \\
\hline & NA & -0.1989 & 0.05924 & & PAV & 0.05375 & 0.009687 \\
\hline & \multicolumn{3}{|c|}{ Scaled deviance $=383.88(732)$} & & PAV2 & -0.0001875 & 0.00005702 \\
\hline & \multicolumn{3}{|c|}{ Null model Scaled deviance $=725.96$} & & MTA & 9.754 & 2.362 \\
\hline & \multirow{2}{*}{\multicolumn{3}{|c|}{4 quadrats removed }} & & MTA2 & -0.3016 & 0.06835 \\
\hline & & & & & PSEAS & 0.08509 & 0.01882 \\
\hline 2.1 & 1 & -2.180 & 0.6309 & & PCLDQ & 0.07075 & 0.02227 \\
\hline & $\mathrm{EC}$ & -0.02220 & 0.002303 & & PCLDQ2 & -0.0001696 & 0.00004756 \\
\hline & $\mathrm{EC}^{2}$ & $4.469 \mathrm{e}-06$ & $8.355 e-07$ & & $\mathrm{~K}$ & -1.530 & 0.4017 \\
\hline & MG & -0.4612 & 0.05543 & & \multicolumn{3}{|c|}{ Scaled deviance $=289.35(736)$} \\
\hline & $\mathrm{MG}^{2}$ & 0.03278 & 0.003389 & & \multicolumn{3}{|c|}{ Null model Scaled deviance $=493.26$} \\
\hline & CA & 0.7759 & 0.08085 & & & & \\
\hline & $\mathrm{CA}^{2}$ & -0.04791 & 0.007760 & 2.8 & 1 & -151.1 & 29.41 \\
\hline & ELCODE & 4.498 & 0.5939 & & $\mathrm{PH}$ & 0.4538 & 0.08856 \\
\hline & $\mathrm{ELCODE}^{2}$ & -1.323 & 0.1620 & & NA & 0.06963 & 0.007764 \\
\hline & PANN & -0.004360 & 0.0006660 & & TAR & -0.6088 & 0.05855 \\
\hline & \multicolumn{3}{|c|}{ Scaled deviance $=601.24(735)$} & & MTDQ & 13.81 & 2.754 \\
\hline & \multirow{2}{*}{\multicolumn{3}{|c|}{ Null model Scaled deviance $=1174.7$}} & & MTDQ2 & -0.3037 & 0.06305 \\
\hline & & & & & SAND & 0.04810 & 0.01649 \\
\hline \multirow[t]{2}{*}{2.2} & \multirow{2}{*}{\multicolumn{4}{|c|}{ Model does not converge }} & \multicolumn{3}{|c|}{ Scaled deviance $=267.84(738)$} \\
\hline & & & & & \multicolumn{3}{|c|}{ Null model Scaled deviance $=570.66$} \\
\hline 2.3 & 1 & -29.93 & 8.610 & & & & \\
\hline & $\mathrm{PH}$ & 6.214 & 2.241 & 2.9 & Model does & onverge & \\
\hline & $\mathrm{PH}^{2}$ & -0.3801 & 0.1448 & & & & \\
\hline & NA & 0.1773 & 0.03431 & & & & \\
\hline & $\mathrm{NA}^{2}$ & -0.002026 & 0.0005424 & 3.1 & 1 & 2.488 & 2.157 \\
\hline & SILT $^{2}$ & -0.01831 & 0.006157 & & $\mathrm{EC}$ & -0.003301 & 0.0003852 \\
\hline & ELCODE & -1.170 & 0.1939 & & $\mathrm{EC}^{2}$ & $9.680 \mathrm{e}-07$ & $2.031 \mathrm{e}-07$ \\
\hline & MTDQ & 0.2234 & 0.07819 & & CLAY & 0.03700 & 0.01531 \\
\hline & Scaled devi & $268.98(737)$ & & & CLAY ${ }^{2}$ & -0.001879 & 0.0006675 \\
\hline & Null model & deviance $=$ & & & PANN & 0.02176 & 0.002488 \\
\hline & & & & & PANN $^{2}$ & $-2.247 \mathrm{e}-05$ & $2.674 \mathrm{e}-06$ \\
\hline 2.4 & 1 & -42.42 & 25.63 & & ELCODE & 2.075 & 0.2513 \\
\hline & $\mathrm{PH}^{2}$ & -0.04831 & 0.009302 & & $\mathrm{ELCODE}^{2}$ & -0.4780 & 0.05077 \\
\hline & MXTWP & -2.424 & 0.8990 & & LONG & -0.07544 & 0.01761 \\
\hline & MXTWP 2 & 0.03275 & 0.01504 & & Scaled devi & $1273.6(735)$ & \\
\hline & MTDQ & 8.103 & 3.021 & & Null model & $d$ deviance $=$ & 3.7 \\
\hline & $\mathrm{MTDQ}^{2}$ & -0.2002 & 0.07117 & & & & \\
\hline & PSEAS & 0.09389 & 0.01303 & 3.2 & 1 & -144.0 & 27.92 \\
\hline & ELCODE & -1.079 & 0.1808 & & $\mathrm{EC}$ & 0.002498 & 0.0003982 \\
\hline & Scaled devia & $177.41(737)$ & & & $\mathrm{EC}^{2}$ & $-1.444 \mathrm{e}-06$ & $3.204 \mathrm{e}-07$ \\
\hline & Null model & deviance $=$ & & & $\mathrm{PH}$ & 0.3007 & 0.04670 \\
\hline & & & & & ISO & 491.5 & 107.4 \\
\hline 2.5 & Model does & nverge & & & $\mathrm{ISO}^{2}$ & -437.5 & 103.5 \\
\hline & & & & & ELCODE & 4.107 & 0.5600 \\
\hline & & & & & ELCODE $^{2}$ & -0.6916 & 0.1022 \\
\hline & & & & & PANN & -0.003993 & 0.0007386 \\
\hline & & & & & Scaled devi & $831.45(736)$ & \\
\hline & & & & & Null model & deviance $=$ & \\
\hline
\end{tabular}


Table 8 (cont.)

\begin{tabular}{|c|c|c|c|}
\hline & parameter & estimate & s.e. \\
\hline \multirow[t]{10}{*}{3.3} & 1 & -12.57 & 1.346 \\
\hline & PH & 2.650 & 0.3181 \\
\hline & $\mathrm{PH}^{2}$ & -0.1585 & 0.02149 \\
\hline & MTCQ & -0.1515 & 0.02357 \\
\hline & PWRMQ & 0.09257 & 0.02259 \\
\hline & $\mathrm{PWRMQ}^{2}$ & -0.001150 & 0.0002250 \\
\hline & ELCODE & 2.367 & 0.1908 \\
\hline & $\mathrm{ELCODE}^{2}$ & -0.4431 & 0.03616 \\
\hline & GYP & -0.01128 & 0.001780 \\
\hline & Scaled devi & $1452.8(736)$ & \\
\hline
\end{tabular}

4.1

$\begin{array}{lrr}1 & -16.41 & 1.294 \\ \mathrm{EC} & 0.002505 & 0.0003557 \\ \mathrm{EC}^{2} & -6.294 \mathrm{e}-07 & 2.820 \mathrm{e}-07 \\ \mathrm{OC}^{2} & -0.7244 & 0.1225 \\ \mathrm{OC}^{2} & 0.02043 & 0.004113 \\ \text { TAR } & 0.3196 & 0.03013 \\ \text { ELCODE } & 3.807 & 0.5954 \\ \text { ELCODE }^{2} & -0.7879 & 0.1161 \\ \text { MTDQ } & 0.1342 & 0.04179\end{array}$

Scaled deviance $=712.36(736)$

Null model Scaled deviance $=1449.2$

4.21

$\begin{array}{lrr}1 & -139.6 & 23.71 \\ \text { PH } & 10.55 & 3.353 \\ \text { PH2 } & -0.8614 & 0.2571 \\ \text { OC } & -4.099 & 0.7936 \\ \text { OC2 } & 0.3063 & 0.05511 \\ \text { P } & -0.01422 & 0.006202 \\ \text { PANN } & 0.5662 & 0.1195 \\ \text { PANN2 } & -0.0008642 & 0.0001822 \\ \text { MTD } & 1.215 & 0.3301\end{array}$

scaled deviance $=169.74(736)$

Null model Scaled deviance $=334.57$

5

$\begin{array}{lrr}1 & -97.27 & 29.46 \\ \text { EC } & -0.006469 & 0.001304 \\ \text { EC }^{2} & 1.782 \mathrm{e}-06 & 6.570 \mathrm{e}-07 \\ \text { ELEV }^{2} & -0.02564 & 0.003804 \\ \text { ELEV }^{2} & 0.00004888 & 9.812 \mathrm{e}-06 \\ \text { MTDQ }^{2} & 9.445 & 2.614 \\ \text { MTDQ }^{2} & -0.2177 & 0.05835 \\ \text { PWRMQ } & -0.08822 & 0.01577 \\ \text { KAV } & 0.001435 & 0.0002209\end{array}$

Scaled deviance $=446.45(736)$

Null model Scaled deviance $=629.97$

\section{1}

$\mathrm{PH}$

$\mathrm{PH}^{2}$

MG

$\mathrm{MG}^{2}$

ISO

ELCODE

$\mathrm{ELCODE}^{2}$

EC

Scaled deviance $=309.53(736)$

Null model Scaled deviance $=918.52$

\section{parameter}

7

EC

$\mathrm{EC}^{2}$

MTWRQ

$\mathrm{MTWRQ}^{2}$

PANN

PANN $^{2}$

ELEV

ELEV $^{2}$

ELCODE

Scaled deviance $=1040,5(735)$

Null model Scaled deviance $=2178.2$

8

1

EC

$E C^{2}$

TAR

$\mathrm{TAR}^{2}$

PANN

PANN $^{2}$

ELCODE

ELCODE $^{2}$

$\mathrm{CA}$

Scaled deviance $=313.61(735)$

Null model Scaled deviance $=966.25$

9

1

$\mathrm{EC}$

$\mathrm{EC}^{2}$

MNTCP

$\mathrm{MNTCP}^{2}$

$\mathrm{MTWRQ}^{2}$

ELCODE

MXTWP

$-42.65$

$-0.01430$

$4.679 \mathrm{e}-06$

5.054

$-0.2787$

$-0.04236$

1.439

1.273

Scaled deviance $=337.09(737)$

Null model Scaled deviance $=695.11$

10

1

TAR

$\mathrm{TAR}^{2}$

PANN

PANN $^{2}$

ELCODE

ELCODE ${ }^{2}$

ISO

Scaled deviance $=408.50(736)$

$\begin{array}{rr}-11.28 & 4.687 \\ -0.4137 & 0.06046 \\ 2.359 & 0.3417 \\ -0.05883 & 0.008007 \\ 0.03384 & 0.004052 \\ .758 \mathrm{t}-05 & 3.070 \mathrm{e}-06 \\ 2.433 & 0.4563 \\ -0.3053 & 0.08855 \\ -53.55 & 7.338\end{array}$

Null model Scaled deviance $=1557.1$

11

$$
1
$$

$\mathrm{MG}$

$\mathrm{MG}^{2}$

MTA

$\mathrm{MTA}^{2}$

PWETP

ELCODE

Scaled deviance $=192.05(735)$

Null model Scaled deviance $=501.28$

4 quadrats removed
8.224

0.002320

$1.126 \mathrm{e}-06$

0.6966

0.01528

0.005589

$4.718 \mathrm{e}-06$

0.7185

0.1251

0.02033
4.775

.0004364

0.4363

0.009712

$2.040 \mathrm{e}-06$

0.001399
$4.196 \mathrm{e}-06$

0.03725 
Table 8 (cont.)

\begin{tabular}{|c|c|c|c|c|c|c|c|}
\hline & parameter & estimate & s.e. & & parameter & estimate & s.e. \\
\hline \multirow[t]{2}{*}{12} & \multirow{2}{*}{\multicolumn{3}{|c|}{ Model does not converge }} & 18 & 1 & -10.57 & 0.8083 \\
\hline & & & & & PH & 1.628 & 0.1815 \\
\hline \multirow[t]{12}{*}{13} & 1 & -21.31 & 2.966 & & $\mathrm{PH}^{2}$ & -0.1188 & 0.01294 \\
\hline & $\mathrm{PH}$ & 2.669 & 0.8677 & & PAV & -0.03910 & 0.003577 \\
\hline & $\mathrm{PH}^{2}$ & -0.2328 & 0.06489 & & PAV $^{2}$ & 0.00009331 & $9.230 \mathrm{e}-06$ \\
\hline & $O C$ & -0.2694 & 0.05855 & & PANN & 0.005758 & 0.001819 \\
\hline & NA & -0.5680 & 0.1075 & & $\mathrm{PANN}^{2}$ & $-1.069 \mathrm{e}-05$ & $2.127 \mathrm{e}-06$ \\
\hline & $\mathrm{NA}^{2}$ & 0.008093 & 0.002074 & & ELCODE & 2.496 & 0.1531 \\
\hline & PANN & 0.04303 & 0.003319 & & $\mathrm{ELCODE}^{2}$ & -0.3223 & 0.02549 \\
\hline & PANN $^{2}$ & $-3.031 \mathrm{e}-05$ & $2.900 \mathrm{e}-06$ & & TAR & 0.08750 & 0.008467 \\
\hline & ELEV & 0.004485 & 0.0006722 & & \multicolumn{3}{|c|}{ Scaled deviance $=2409.5(735)$} \\
\hline & \multicolumn{4}{|c|}{ Scaled deviance $=766.26(736)$} & Null model & $\mathrm{d}$ deviance $=$ & \\
\hline & \multicolumn{7}{|c|}{ Null model Scaled deviance $=1594.4$} \\
\hline & & & & 19 & 1 & -200.7 & 40.98 \\
\hline \multirow[t]{13}{*}{14} & 1 & -23.76 & 3.057 & & $\mathrm{EC}$ & -0.002620 & 0.0004459 \\
\hline & PH & 3.310 & 0.9452 & & LAT & 21.65 & 4.089 \\
\hline & $\mathrm{PH}^{2}$ & -0.3054 & 0.07279 & & LAT2 & -0.3670 & 0.06884 \\
\hline & $\mathrm{OC}$ & -0.5194 & 0.04513 & & MTWRQ & -10.38 & 1.841 \\
\hline & $\mathrm{OC}^{2}$ & 0.01367 & 0.001420 & & MTWRQ2 & 0.2113 & 0.03526 \\
\hline & SILT & 0.4384 & 0.04286 & & ELCODE & 2.851 & 0.4614 \\
\hline & $\mathrm{SILT}^{2}$ & -0.01428 & 0.003012 & & ELCODE2 & -0.3709 & 0.07632 \\
\hline & PANN & 0.04345 & 0.002808 & & TAR & 0.1622 & 0.06864 \\
\hline & $\mathrm{PANN}^{2}$ & $-3.207 \mathrm{e}-05$ & $2.441 \mathrm{e}-06$ & & Scaled devi & $539.49(736)$ & \\
\hline & ELEV & 0.005904 & 0.0006169 & & Null model & d deviance $=$ & \\
\hline & \multicolumn{7}{|c|}{ Scaled deviance $=712.61(735)$} \\
\hline & \multirow{2}{*}{\multicolumn{3}{|c|}{ Null model Scaled deviance $=1920.6$}} & 20 & 1 & -3.707 & 0.8821 \\
\hline & & & & & OC & 1.992 & 0.2143 \\
\hline \multirow[t]{9}{*}{15} & 1 & -44.50 & 11.22 & & $O C^{2}$ & -0.3365 & 0.06487 \\
\hline & $\mathrm{EC}$ & -0.08669 & 0.01221 & & $\mathrm{P}$ & -0.01413 & 0.002148 \\
\hline & $\mathrm{EC}^{2}$ & 0.00002819 & $4.750 \mathrm{e}-06$ & & $\mathrm{P}^{2}$ & 0.00001274 & $5.912 \mathrm{e}-06$ \\
\hline & OC & 2.084 & 0.2261 & & PWETP & -0.03146 & 0.003592 \\
\hline & SAND & 1.156 & 0.2653 & & ELCODE & 2.433 & 0.5493 \\
\hline & $\mathrm{SAND}^{2}$ & -0.007352 & 0.001558 & & ELCODE $^{2}$ & -0.2177 & 0.08778 \\
\hline & $\mathrm{N}$ & -34.50 & 4.991 & & MG & -0.2346 & 0.04624 \\
\hline & \multicolumn{4}{|c|}{ Scaled deviance $=239.76(738)$} & \multicolumn{3}{|c|}{ Scaled deviance $=890.84(736)$} \\
\hline & \multicolumn{4}{|c|}{ Null model Scaled deviance $=725.26$} & \multicolumn{3}{|c|}{ Null model Scaled deviance $=1992.9$} \\
\hline \multirow[t]{13}{*}{16} & 1 & -8.174 & 0.7886 & 21 & 1 & -77.65 & 12.06 \\
\hline & $\mathrm{EC}$ & -0.005733 & 0.0007151 & & KAV & 0.004062 & 0.0004572 \\
\hline & $\mathrm{EC}^{2}$ & $1.858 \mathrm{e}-06$ & $3.493 \mathrm{e}-07$ & & $\mathrm{KAV}^{2}$ & $-1.698 \mathrm{e}-06$ & $3.289 \mathrm{e}-07$ \\
\hline & ELCODE & 2.642 & 0.3710 & & MXTWP & 4.742 & 0.7406 \\
\hline & $\mathrm{ELCODE}^{2}$ & -0.2906 & 0.06158 & & MXTWP 2 & -0.07582 & 0.01153 \\
\hline & SILT & 0.2416 & 0.03814 & & PANN & -0.007426 & 0.001465 \\
\hline & $\mathrm{SILT}^{2}$ & -0.01244 & 0.003514 & & ELCODE & 3.082 & 0.5610 \\
\hline & PANN & 0.01418 & 0.002512 & & $\mathrm{ELCODE}^{2}$ & -0.3847 & 0.09297 \\
\hline & $\mathrm{PANN}^{2}$ & $-1.304 \mathrm{e}-05$ & $2.669 e-06$ & & EC & -0.003115 & 0.0005432 \\
\hline & PAV & -0.08403 & 0.01570 & & Scaled devia & $671.15(736)$ & \\
\hline & Scaled devia & $1016.7(735)$ & & & Null model & $d$ deviance $=$ & \\
\hline & Null model & deviance $=$ & & & & & \\
\hline & & & & 22 & 1 & 140.7 & 22.81 \\
\hline 17 & 1 & -15.18 & 0.9080 & & LONG & -1.302 & 0.2014 \\
\hline & $\mathrm{EC}$ & -0.005143 & 0.0005818 & & PWRMQ & 0.06192 & 0.02255 \\
\hline & $\mathrm{CA}$ & 0.2094 & 0.03395 & & ELCODE & 1.850 & 0.2561 \\
\hline & $\mathrm{CA}^{2}$ & -0.01275 & 0.002653 & & Scaled devia & $145.23(741)$ & \\
\hline & TAR & 0.3213 & 0.01873 & & Null model & d deviance $=2$ & \\
\hline & ELCODE & 4.848 & 0.4759 & & & & \\
\hline & $\mathrm{ELCODE}^{2}$ & -0.6012 & 0.07450 & & & & \\
\hline & $\mathrm{PH}$ & -0.3048 & 0.03982 & & & & \\
\hline & Scaled devia & $916.93(737)$ & & & & & \\
\hline & Null model & deviance $=3$ & & & & & \\
\hline
\end{tabular}


lakes and a few coastal lagoons. The model explained $52.6 \%$ of the scaled deviance of the null model. Model terms reflect the known habitat preferences of the assemblage although several of the taxa are undescribed and poorly collected (inland distribution - high precipitation seasonality, gypsum present or very high, exchangeable magnesium moderate, very low organic carbon) (Tables 8 and 9).

Assemblage 2.7 showed little relationship between species composition and richness and poor fit to the Poisson error model. The assemblage's taxa occurred in species poor quadrats and inspection of the full two-way table revealed some distinct subgroups within the assemblage. The fitted model explained only $41.3 \%$ of the scaled deviance of the null model. Climate terms dominated the model and were consistent with the assemblage's distribution predominantly in the western Avon Wheatbelt bioregion (i.e. low to moderate annual mean temperature and moderate precipitation in the coldest quarter, Table 8).

Assemblage 2.8 showed a weak unimodal relationship between composition and species richness. The Poisson fit was acceptable and model explained $53.1 \%$ of the scaled deviance of the null model. This assemblage showed maximum species richness in coastal salt lakes, saline riverbanks and seeps (moderate to high exchangeable sodium and high $\mathrm{pH}$ ). The inclusion of the model term mean temperature of driest quarter is consistent with the near coastal distribution of the assemblage's component taxa (Table 8 and 9). The assemblage was mostly recorded along the south coast, but all of the component taxa, with the exception of Velleia exigua and to a lesser extent Triglochin striata, have distributions that extend to coastal parts of the Geraldton Sandplains bioregion.

Assemblage 2.9 was considered an artifact and modeling iterations did not converge.

Assemblage 3.1 showed a strong unimodal relationship between composition and species richness. Species richness was taken as a useful surrogate for composition. The model however explained only $26.1 \%$ of the null model scaled deviance (Table 9). The assemblage represents a very widespread group of wetland and dampland taxa. Despite low explanatory power of the model, individual model terms were generally consistent with the known habitat preferences and distribution of the assemblage's taxa. The assemblage was richest on the damp margins of wetlands (intermediate wetland elevations) with low salinity (including some specific low salinity substrates within saline wetlands). The assemblage is centred within the wheatbelt and mesic South West and this is reflected by the inclusion of annual precipitation and longitude in the model (Table 8).

Assemblage 3.2 showed a strong unimodal relationship between composition and species richness. Species richness was therefore regarded as a reasonable surrogate for composition. The model explained $36.5 \%$ of the null model scaled deviance (Table 9). Despite the low explanatory power of the model, individual model terms were generally consistent with the known habitat preferences and distribution of the assemblage's taxa. The assemblage showed maximum richness in saline wetlands (moderate to high EC), in coastal and southern near coastal wetlands (high isothermality). The known occurrence of the assemblage's taxa on berms and flats (including evaporites) is reflected by inclusion of the terms EC, pH (high) and ELCODE (intermediate wetland elevations) in the model.

Assemblage 3.3 The relationship between the one-dimensional ordination and assemblage species richness was very strongly unimodal and suggested that species richness was a good surrogate for assemblage composition. The model explained only $44 \%$ of the total scaled deviance. This assemblage was richest on wetland margins (not basins or elevated dunes) with maximum richness at ELCODE 2 and 3 (Table 8). Percentage gypsum was a significant term in the GLM model. Richness was constrained at extremely high gypsum content $(>70 \%)$ rather than being strongly negatively related. The fitted value of the model showed that $\mathrm{pH}$ was negatively correlated with richness at low values $(<6.5)$, and that richness was high yet variable at $\mathrm{pH} 7.5$ to 9 (Table 8 ). Model terms were consistent with the known habitats of the assemblage's component species. Most members are widespread around the edges of a variety of saline and brackish wetlands. These strandlines/beaches and low dunes are often rich in evaporites that have high $\mathrm{pH}$ due to the presence of $\mathrm{Na}, \mathrm{Mg}$ and $\mathrm{Ca}$ carbonates. While the assemblage occurred widely throughout the study area with variable richness, species rich quadrats were located within a central band (low mean temperature coldest period and low precipitation in the warmest quarter). The assemblage occurred at ca. $72 \%$ of quadrats included in the modeling and several taxa were the most frequent taxa in the survey (Parapholis incurva, Triglochin mucronata). This may account for the low explanatory power of the GLM model (Table 9).

Assemblage 4.1 The assemblage showed a strong unimodal relationship between species richness and composition. The model explained $51 \%$ of the total scaled deviance (Table 9). The significant terms in the GLM model were generally consistent with the known distribution and habitat preferences of the assemblage's taxa. The assemblage occurs on the margins of primary saline wetlands reflected by the inclusion ELCODE (maximum richness at ELCODE 2 and 3 ) and EC (maximum richness $300-1000 \mathrm{mS}$ $\mathrm{m}^{-1}$ ) in the model. Soils on the margins of saline 
Table 9 Summary of the performance of Generalized Linear Models (GLM) for 34 wetland species assemblages. Modality refers to the relationship between assemblage richness and species composition based on a plot of species richness against a 1D-SSH ordination. Assemblages are sorted into blocks based on modality, and conformance with model assumptions. For five assemblages modeling iterations did not converge.

\begin{tabular}{|c|c|c|c|c|c|c|c|c|}
\hline assemblage & modality & $\begin{array}{l}\text { No. } \\
\text { taxa }\end{array}$ & $\begin{array}{c}\text { No. } \\
\text { quadrats }\end{array}$ & $\begin{array}{l}\text { Max. } \\
\text { quadrat } \\
\text { richness }\end{array}$ & $\begin{array}{c}\text { Total } \\
\text { scaled } \\
\text { deviance }\end{array}$ & $\begin{array}{c}\text { model } \\
\text { scaled } \\
\text { deviance }\end{array}$ & $\begin{array}{c}\text { Poison } \\
\text { fit }\end{array}$ & $\begin{array}{c}\text { Scaled } \\
\text { deviance } \\
\text { explained }(\%)\end{array}$ \\
\hline 1.2 & unimodal & 43 & 90 & 7 & 725.96 & 383.88 & Moderate & 47.1 \\
\hline 2.1 & unimodal & 42 & 115 & 10 & 1174.7 & 601.24 & Moderate & 48.8 \\
\hline 2.8 & ?unimodal & 10 & 72 & 5 & 570.66 & 267.84 & Moderate & 53.1 \\
\hline 3.1 & unimodal & 19 & 398 & 10 & 1723.7 & 1273.6 & Good & 26.1 \\
\hline 3.2 & unimodal & 21 & 203 & 12. & 1309.5 & 831.45 & Good & 36.5 \\
\hline 3.3 & unimodal & 23 & 546 & 13 & 2202.6 & 1452.8 & Good & 44.0 \\
\hline 4.1 & unimodal & 24 & 208 & 10 & 1449.2 & 712.36 & Good & 50.0 \\
\hline 5 & unimodal & 23 & 73 & 9 & 629.97 & 446.45 & Moderate & 29.1 \\
\hline 6 & unimodal & 40 & 74 & 12 & 918.52 & 309.53 & Moderate & 66.3 \\
\hline 7 & unimodal & 34 & 355 & 15 & 2178.2 & 1040.5 & Good & 52.2 \\
\hline 9 & unimodal & 16 & 76 & 7 & 695.11 & 337.09 & Moderate & 61.5 \\
\hline 10 & unimodal & 80 & 78 & 23 & 1557.1 & 408.5 & Moderate & 73.8 \\
\hline 13 & ?unimodal & 46 & 106 & 22 & 1594.4 & 766.26 & Moderate & 51.9 \\
\hline 14 & unimodal & 50 & 130 & 21 & 1920.6 & 712.61 & Moderate & 62.9 \\
\hline 16 & unimodal & 24 & 259 & 13 & 1893.6 & 1016.7 & Good & 46.3 \\
\hline 17 & unimodal & 50 & 234 & 22 & 3393.2 & 916.93 & Good & 73.0 \\
\hline 18 & unimodal & 68 & 536 & 32 & 6443.8 & 2409.5 & Good & 62.6 \\
\hline 19 & unimodal & 64 & 163 & 18 & 1955.5 & 539.49 & Good & 72.4 \\
\hline 20 & unimodal & 77 & 187 & 15 & 1992.9 & 890.84 & Good & 63.3 \\
\hline 21 & unimodal & 36 & 142 & 12 & 1278 & 671.15 & Good & 47.5 \\
\hline 2.3 & unimodal & 18 & 60 & 3 & 405.09 & 268.98 & Poor & 33.6 \\
\hline 2.6 & unimodal & 10 & 37 & 3 & 311.41 & 147.65 & Poor & 52.6 \\
\hline 4.2 & ?unimodal & 10 & 26 & 5 & 334.57 & 169.74 & Poor & 49.3 \\
\hline 8 & ?unimodal & 33 & 62 & 18 & 966.25 & 313.61 & Poor & 67.5 \\
\hline 11 & ?unimodal & 24 & 37 & 10 & 501.28 & 192.05 & Poor & 61.7 \\
\hline 15 & unimodal & 28 & 38 & 24 & 725.26 & 239.76 & Poor & 66.9 \\
\hline 22 & ?unimodal & 12 & 17 & 8 & 253.62 & 145.23 & Very poor & 42.7 \\
\hline 2.4 & no relationship & 17 & 61 & 5 & 526.85 & 177.41 & Moderate & 66.3 \\
\hline 2.7 & no relationship & 9 & 61 & 5 & 493.26 & 289.35 & Poor & 41.3 \\
\hline 1.1 & no relationship & 8 & 11 & 4 & 167.15 & No convergence & Very poor & - \\
\hline 2.2 & no relationship & 7 & 6 & 7 & 153.77 & No convergence & Very poor & - \\
\hline 2.5 & no relationship & 4 & 8 & 3 & 112.27 & No convergence & Very poor & - \\
\hline 2.9 & no relationship & 4 & 8 & 2 & 101.06 & No convergence & Very poor & - \\
\hline 12 & no relationship & 6 & 8 & 6 & 126.76 & No convergence & Very poor & - \\
\hline
\end{tabular}

playas and braided channels are often of recent origin with little vegetation biomass and this is reflected in the strong negative relationship between assemblage richness and soil organic carbon. Maximum species richness occurred in the north-east of the study area consistent with the inclusion of annual temperature range and mean temperature of the driest quarter in the model (Table 8).

Assemblage 4.2 showed a weak unimodal relationship between species richness and composition. Given the small number of taxa and occurrences across the data set (Table 9), initially only four-term linear models were examined. The four-term model only explained $33.7 \%$ of the scaled deviance of the null model (Table 9) and the Poisson fit was poor. The model was sensitive to several quadrats but their removal did not alter the significance of model terms. The taxa in this assemblage were uncommon and occupied a distinct habitat of low sandy berms and pan margins. The terms of the simple model were consistent with this leached sand substrate (low organic carbon, low available phosphorus). The occurrence of this assemblage in the northern and central wheatbelt is consistent with the climatic terms of the model (mean diurnal temperature range and precipitation of the wettest period). Fitting of polynomials improved explanatory power although the Poison fit was poor and the model was sensitive to several quadrats. Removal of influential quadrats did not alter the significance of model terms and the model 
was considered to be robust. The polynomial model explained $49 \%$ of the total scaled deviance. The polynomial shared the terms organic carbon and mean diurnal temperature range with the four-term model and total $P$ replaced available phosphorus. The assemblage showed maximum richness at intermediate $\mathrm{pH}$ (Table 8). This is consistent with the assemblage occurring on seasonally leached sands rather than evaporite derived substrates (cf. assemblage 3.3)

Assemblage 5 showed a strong unimodal relationship between richness and composition, as derived from a one-dimensional ordination, and conformed to model assumptions. Explanatory power of the model however was very poor $29.1 \%$, Table 9). The soil parameter terms of the model were consistent with the preference of the assemblage's taxa for low salinity sites on finer textured soils (low EC and moderate levels of available potassium, Table 8). Quadrats with high richness for the assemblage were few and showed little geographic patterning (Figure 10). This may account for the poor explanatory power of the model (Table 9)

Assemblage 6 showed a strong unimodal relationship between species richness and composition, as determined from a onedimensional ordination. Poison fit was moderate and the model explained $66.3 \%$ of the total scaled deviance (Table 9). The assemblage shows maximum richness on sandplain soils (moderate but variable $\mathrm{pH}$, low $\mathrm{Mg}$ and low $\mathrm{EC}$, intermediate to high ELCODE, Table 8). Although some members of the assemblage have broader distributions, the assemblage showed maximum species richness along the south coast and this is consistent with the high isothermality reported by the model (Table 8).

Assemblage 7 showed a strong unimodal relationship between species richness and composition. Poisson fit was good and the model explained $52.2 \%$ of the scaled deviance of the null model (Table 9). Although some members of the assemblage occupy brackish/saline wetlands, maximum richness of the assemblage is associated with non-saline wetlands and substrates (low EC, Table 8). The southwestern and near coastal distribution of the assemblage is also consistent with the model terms (moderate annual precipitation, low to moderate elevation above sea level, Table 8). The assemblage was poorest within wetland basins and this was consistent with the inclusion of ELCODE in the model.

Assemblage 8 was a heterogeneous group of taxa that showed a weak unimodal or possibly bimodal relationship between species richness and composition. The relevant GLM model explained $67.5 \%$ of the total scaled deviance. The few species rich quadrats were localized on the south-east coast of the study area and this may account for the explanatory power of the model (associated with climatic model terms). The taxa were all terrestrial species and not comprehensively sampled during the current survey. Despite this the terms of the model were partially consistent with the broader distribution and habitat preferences of the assemblage's taxa. The assemblage shows maximum richness in coastal and near coastal areas of the south-west (low/intermediate annual temperature range, high/moderate annual rainfall). The assemblage's terrestrial species did not occur in the basins of wetlands (moderate to high ELCODE). The preference by the majority of the taxa for sandy coastal soils is consistent with the model terms (EC and exchangeable calcium) (Table 8).

Assemblage 9 showed a strong unimodal relationship between species richness and composition, Poisson fit was acceptable and the model explained $61.5 \%$ of the total scaled deviance. The model terms were only partially consistent with the assemblage's broader geographic range and habitat preferences. The taxa show maximum species richness on granite aprons and derived soils, including sandy lunettes (elevated sites, very low salinity soils). They occur much more widely in terrestrial habitats than sampled in the current survey. The climatic terms of the model were only partially consistent with the broader distribution of the assemblage's taxa.

Assemblage 10 showed a strong unimodal relationship between species richness and composition, Poisson fit was moderate and the model explained $73.3 \%$ of the scaled deviance of the null model. Species richness was considered a good surrogate for composition in this group. This assemblage contains both terrestrial and wetland restricted taxa that occur as components of riparian zones in streams, rivers, non-saline lakes and sedge swamps. The model terms are generally consistent the broader distribution and habitat preferences of the assemblage. Quadrat richness was maximum in non-saline riparian sites (low exchangeable sodium, moderate ELCODE, Table 8) centred within the Jarrah Forest and adjacent coastal bioregions (low/ moderate annual temperature range, moderate/high annual precipitation, Table 8).

Assemblage 11 showed no relationship between species composition and species richness and a poor fit to the Poisson error model. The assemblage includes small numbers of taxa, recorded at few quadrats in the south-west of the study area. They occur in riparian zones of fresh wetlands. Four quadrats were removed to achieve a stable model and model terms were difficult to interpret yet the model explained $61.7 \%$ of the total scaled deviance. The component taxa include a very heterogeneous group distributed in coastal and intermediate rainfall parts of the south-west. 
Assemblage 12 contained only six taxa recorded from eight quadrats. Only two quadrats were species rich and the modeling iterations did not converge. The taxa are poorly known but, based on the current survey, they occupy similar habitats to assemblage 4.2 .

Assemblage 13 displayed a weak unimodal relationship between species richness and composition, while the distribution of the data conformed to the Poisson model. The model explained $51.9 \%$ of the total scaled deviance and model terms were generally consistent with the known distribution patterns of the assemblage's taxa (Figure 10, Table 8). The assemblage maximum species richness was associated with non-saline wetland and terrestrial habitats with fine textured soils (low exchangeable sodium, intermediate $\mathrm{pH}$, low organic carbon, Table 8).

Assemblage 14 showed a strong unimodal relationship between species richness and composition. Species richness was considered a good surrogate for composition in the assemblage. The data conformed to the model assumptions and the model explained $62.9 \%$ of the total scaled deviance (Table 9). The model terms strongly reflected the assemblage's occurrence in claypans basins and marginal clay flats in the south-west of the study area. Maximum assemblage species richness occurs at quadrats with low to moderate soil $\mathrm{pH}(\mathrm{pH} 5.5-7)$, low soil organic carbon and relatively high soil silt fraction (fine textured) and high annual precipitation (Figure 10, Table 8 ).

Assemblage 15 The plot of richness against a onedimensional ordination was strongly unimodal and species richness was regarded as a good surrogate for composition. The data however violated model assumptions, as the Poisson fit was poor. The distribution of assemblage richness values was very skewed, with two very species rich quadrats and the remaining 36 quadrats very species poor. Despite being sensitive to multiple quadrats, their removal did not alter the significance of model terms. The model terms were only partially consistent with the broader habitat preferences of the component taxa. The assemblage is most species rich in high rainfall sedge swamps, winter-wet flats and claypans (low EC and moderate sand content equating to finer texture, Table 8).

Assemblage 16 showed a very strong relationship between species richness and composition based on a one-dimensional ordination plotted against species richness. The Poisson fit was good and the model explained $46.3 \%$ of the scaled deviance of the null model. The model terms were generally consistent with the broader distributions of the component taxa. Although generally widespread, maximum richness was centred in the Avon Wheatbelt and western margin of the Jarrah Forest bioregions (intermediate annual rainfall) on granitic and related soils (low salinity, relatively high silt content at ca. 10\%). Around wetlands maximum richness occurred on dunes and flats (high wetland elevations), consistent with the assemblage being dominated by terrestrial taxa (Table 8, Figure 10).

Assemblage 17 showed a very strong unimodal relationship between species richness and composition. The Poisson fit was good and the model explained $73.0 \%$ of the total scaled deviance (Table 9). The model terms strongly reflect the habitat and distribution preferences of the assemblage. This assemblage occupies a similar landscape position to assemblage 16 but has a distinctly northeastern centre of distribution (Figure 10). Maximum species richness occurred in the north east of the study area (high annual temperature range) on granitic soils (low EC, low to intermediate $\mathrm{pH}$, with very low to low exchangeable calcium, Table 8 ). The taxa are not associated with the basins and immediate edges of wetlands (moderate to high elevations, Table 8).

Assemblage 18 showed a very strong relationship between species richness and composition. The Poisson fit was good and the model explained $62.6 \%$ of the null model scaled deviance. The climatic terms of the model terms strongly reflected the inland distribution of the assemblage's taxa (low annual precipitation, high annual temperature range, Table 8). The predominance of terrestrial taxa within the assemblage is consistent with the inclusion of the model term ELCODE (Table 8). The edaphic terms of the model are more difficult to interpret. Maximum species richness occurred over a range of $\mathrm{pH}$ values between 6 and 8 . This is a relatively large range (cf. assemblage 17) and may reflect the broad array of habitats occupied by the assemblage's taxa.

Assemblage 19 showed a strong relationship between species richness and composition and conformed to model assumptions. The GLM model explained $72.4 \%$ of the total scaled deviance (Table $9)$. The climatic terms of the model, high mean temperature in the warmest quarter and high annual temperature range, coupled with latitude, strongly reflect the strong northeastern arid and semi-arid distribution of the assemblage (Table 9, Figure 10). The component taxa occur in a range of habitats yet are essentially absent from wetland basins and edges subject to inundation, (moderate to high ELCODE) and evaporites and derived soils (low EC, Table 9). Within wetland habitats the assemblage occurred within shrublands on elevated rises as well as mid slope chenopod dominated low shrublands on the margins of salt lakes.

Assemblage 20 showed a strong unimodal relationship between species richness and composition and conformed to the Poisson error model. Species richness of the assemblage was regarded as a good surrogate for composition. The 
model describes $63.3 \%$ of the scaled deviance of the null model (Table 9). The model terms are generally consistent with the distribution and habitat preferences of the taxa. Maximum species richness occurred in the Avon wheatbelt and Mallee bioregions (low precipitation in the wettest period; Table 8, Figure 10). The taxa were associated with valley bottoms and wetland features such as sandy lunettes and flats (low/moderate organic carbon, low to moderate total phosphorus, elevated positions relative to wetlands and low exchangeable magnesium, Table 8).

Assemblage 21 showed a strong unimodal relationship between species richness and composition and conformed to the Poisson error model. The model explained $47.5 \%$ of the scaled deviance of the null model. The model terms are consistent with the maximal richness of the assemblage occurring at valley floor sites often associated with salt lake systems with finer textured soils (low to moderate EC, high wetland elevations, moderate available potassium; Table 8). The climatic terms of the model reflect the assemblage's maximum richness within the southeastern Avon Wheatbelt and Mallee bioregions (moderate maximum temperature wettest period, low annual rainfall; Table 8).

Assemblage 22 was a small heterogeneous group of taxa that occurred at very few quadrats. Little relationship was observed between composition and species richness and the Poisson fit of the data was very poor. The explanatory power of the model was ca. $43 \%$, largely the product of the localized distribution of the species rich quadrats (Figure, 9). While the group's taxa tend to have a northern centre of distribution, the model terms latitude and precipitation in the warmest quarter reflect the sampling rather then the broader distributions of the component taxa. The positive relationship with elevation (ELCODE), is consistent with the assemblage's taxa being wholly terrestrial and not associated with the basins or immediate margins of wetlands (Table 8).

\section{DISCUSSION}

Prior to the current study no broad scale quadrat based floristic survey of the wetlands of the wheatbelt had been undertaken. A total of 1436 taxa were recorded from the 813 quadrats sampled. This is a significant number given the narrow range of habitats sampled (at the landscape scale) compared to the survey of terrestrial quadrats by Gibson et. al (2004), which reported 2609 taxa from 682 quadrats. Eight newly recognized taxa were collected during the current survey and collections were made of 78 conservation taxa (Appendix 3). The dominant families recorded are similar to those recorded in SWBP as a whole (Beard et al., 2000), although the relative importance of families is very different Asteraceae, Chenopodiaceae, Poaceae and Apiaceae are well represented in wetland habitats compared with the remainder of the landscape (cf. Beard et al., 2000; Gibson et al., 2004).

The subfamily Salicornioideae of the Chenopodiaceae are almost exclusively halophytic and the richness of this subfamily recorded within the wheatbelt reflects the intensive sampling of saline habitats. A taxonomic revision of this subfamily is still to be completed but currently it is considered to be comprised of roughly 90 species in ca. 15 genera. Of these 41 species are recorded from Australia and all but one species and two subspecies are endemic to Australia (Kühn et al., 1993; K. Sheppard, pers comm). Within the subfamily the genus Halosarcia is particularly speciose (ca. 28 species) and several new taxa were discovered during the survey (Appendix 3). The wheatbelt and adjacent arid zone are the centre of species diversity for the genus (Wilson, 1980; Kühn et al, 1993).

Members of the Asteraceae, particularly annuals, are recognized as being a prominent feature of the woodlands of the wheatbelt (Keighery et al., 2000; Beard et al., 2000; Gibson et al., 2004). Elements of the Asteraceae (e.g. Angianthus sensu lato, Epitriche, Haegiela and Gnephosis) have also shown significant radiation at the generic and species level within saline environments particularly in south-west Western Australia (Short, 1982; Keighery, in press a).

The Apiaceae recorded during the survey occupied a variety of habitats and were dominated by Hydrocotyle (14 taxa) and Eryngium (six taxa) with Apium and Neosciadium also being prominent. Hydrocotyle was recorded in a broader array of habitats. Of particular note is a suite of species associated with naturally saline wetlands (Hydrocotyle hexaptera ms, $H$. coorowensis ms, $H$. medicaginoides, and H. sp Truslove (M.A. Burgman 4419)). Eryngium was associated with non-saline wetlands in the higher rainfall parts of the study area (see assemblages 14 and 15 and quadrat group 20; Appendix 4). Keighery (in press b) recognises seven taxa of Eryngium in Western Australia: six are associated with clay based wetland habitats and the seventh (Eryngium pinnatifidum subsp. pinnatifidum) generally occurs in coastal non-wetland habitats between Geraldton and Albany. The Eryngium not recorded during the current survey, Eryngium subdecumbens, is a rare taxon restricted to the clay based wetlands of the Swan Coastal Plain. Members of the genus occupy similar habitats in eastern Australia (Michael, 1999) and California (Hickman. 1993).

Several other groups have centres of diversity in the wetlands of the wheatbelt. For annual members of the genus Triglochin (Juncaginaceae) the wetlands of the study area are a world centre of diversity 
(Keighery and Keighery, 2003). They occur in damp habitats, commonly in multi-species stands on the margins of naturally saline wetlands. Other damp habitat specialists such as annual Cyperaceae (Schoenus, Isolepis), Drosera, annual Stylidium and Centrolepis are also prominent genera within the wetlands of the study area. These genera are abundant in fresh, seasonally wet habitats throughout the south-west, particularly in the higher rainfall zone (Gibson et al., 1994; Gibson and Keighery, 2000). Like Triglochin, members of these genera many also occur in damp areas on the margins of inland saline wetlands (e.g. Drosera salina, D. zigzagia, Centrolepis eremica, C. humillima, Isolepis polygyna and Stylidium sp. aff. obtusatum (M.N. Lyons 2926).

The genus Frankenia was also a major component of the flora of coastal and inland saline wetlands. The genus is in great need of taxonomic revision and the identity of many specimens was difficult to determine. Due in part to the poor state of the taxonomy, the group is poorly collected. Collections of presumed extinct and previously unrecognised taxa were made during the survey. The Frankeniaceae includes four genera and ca. 100 species worldwide. They are distributed in temperate and sub-tropical regions and are mostly halophytes of coastal and arid inland habitats (Barnsley, 1982; Watson and Dallwitz, 2000). In Australia the family is represented by 47 species of Frankenia and the SWBP contains $36 \%$ of these. Further taxonomic work is likely to increase the number of taxa known from the SWBP.

The representation of Acacia, Melaleuca, Eucalyptus and Stylidium in wetlands parallels their importance in the SWBP as a whole (Beard et al., 2000; Gibson et al., 2004). Several taxa of Melaleuca (M. strobophylla and $M$. atroviridis $\mathrm{ms}$ ) and Eucalyptus (E. occidertalis and $E$. camaldulensis) are structural dominants of wetlands in the study area.

The wetlands of the wheatbelt are rich in herbaceous annuals and poor in shrub species, compared to the wheatbelt landscape as a whole (Gibson et al., 2004) although herbs are an important component of woodland vegetation. Both woodlands and many wetland habitats are open and provide gaps that can be colonised by annual herbs. Herbaceous annuals represented $29.3 \%$ of the total wetland flora recorded (1436 taxa) and this proportion increased at lower wetland elevations (Table 2). Shrubs comprised $31.5 \%$ of the wetland flora, with the proportion increasing at higher elevations (Table 2).

Aquatic and amphibious taxa were $9.6 \%$ of the flora recorded (51 aquatic and 88 amphibious taxa) for the study area (Appendix 1) and sites with low EC were the richest in these taxa (Figure 6). The definition of aquatic as used here is narrower than employed by Jacobs and Wilson (1996) in an evaluation of the freshwater plants of Australasia. They regarded the wetland flora of the south-west of Australia (effectively the SWBP, Beard, 1980), as biogeographically distinct, with high levels of endemism. Speciation in genera such as Villarsia, Isoetes and Utricularia has been noted as distinguishing the south-west freshwater aquatic flora from other parts of Australia (Jacobs and Wilson, 1996).

The more extensive sampling in the current survey suggest the wetland flora of the SWBP is less biographically distinctive than previously thought (Jacobs and Wilson, 1996). Our data shows three biogeographical groups within the aquatic and amphibious taxa. The first group was dominated by species with distributions centred in the higher rainfall south-west and coastal parts of the study area as suggested by Jacobs and Wilson (1996). A second group comprising taxa such as Wurmbea murchisoniana, Peplidium sp. C. (N.T. Burbidge \& A. Kanis 8158), Elatine macrocalyx and Juncus aridicola have distributions centred in the semi-arid and arid zone. The third group, including Ottelia ovalifolia, Damasonium minus, Marsilea mutica and $M$. drummondii, represents a widespread element whose distributions extend into central and northern Australia. The wetland flora of the SWBP, while having a south-west element, cannot be considered uniquely south-western.

\section{Quadrat analysis}

The quadrat classification primary division largely corresponded to the separation of quadrats of inland naturally saline and some secondarily saline wetlands from a heterogeneous group of predominantly fresh swamps, fresh and saline sedgelands, claypans and wooded swamps distributed largely in the western and southern parts of the study area (Figure 7). Coastal saline wetland margins also fell in the latter half of the dendrogram. The quadrat ordination strongly reflected the primary division of the dendrogram and the ordination was strongly correlated with temperature and rainfall vectors.

At the 26 and 39-group level most quadrat groups showed strong geographic patterning (Table 3). Quadrats groups of lunettes and inter-wetland flats showed strong geographic patterning (groups $1.2-$ southern, 1.3 - northern/central and 1.4 - western). The lower margins of inland salt lakes also showed strong geographic patterning, with groups 6 and 9.2 having discreet southern distributions. In addition communities of gypsum beds also show a strong northern and southern change in species composition (quadrat group 3 cf. group 6). Similarly fresh wetland quadrat groups showed strong geographic patterning, with inland (groups 16 and 17) and southwestern clusters (groups 19, 20 and 21). The geographic patterning was poorly reflected in 
the IBRA subregional occurrences for quadrat groups (Table 3). Only two small quadrat groups were restricted to a single IBRA region or sub-region (groups 6 and 24), although groups did tend to occur in adjacent regions and sub-regions. Major catchments also showed little one to one correlation with quadrat groupings (Table 5). These results suggest that neither IBRA or major catchments are particularly useful tools for conservation planning for the wetland vegetation of the study area.

At the 39-group level quadrat groups showed strong patterning related to edaphic factors (EC, $\mathrm{pH}$ and the presence of gypsum) and local elevation (Table 4, Figure 8).

\section{Species classification and assemblage modeling}

Twenty-nine models could be constructed from the 34 assemblages identified from the cluster analysis. For five assemblages, modeling iterations did not converge and models could not be constructed. Three of these (assemblages 1.1, 2.2 and 2.9) were regarded as artifactual and represented very few terrestrial taxa recorded from few quadrats. The remaining two assemblages 2.5 and 12) also had very few taxa yet were regarded as being ecologically coherent and well sampled. They were small clusters of species restricted to very discrete wetland habitats.

Twenty assemblages showed an acceptable unimodal relationship between species richness and a one-dimensional ordination of species composition and conformed to model assumptions (Table 9). The explanatory power of these models ranged from $26.1 \%$ to $73.8 \%$. Of these, 17 of models were generally consistent with the broader habitat preferences and distributions of their component taxa and were regarded as robust. Models that were highly consistent with known habitats and distributions did not necessarily have the greatest explanatory power. The model for assemblage 3.1 explained only $26.1 \%$ of the total scaled deviance, although the assemblage was well sampled during our study. This assemblage was ubiquitous at lower salinity damp/wet habitats, at a wide variety of wetlands. Many of the taxa in the assemblage were very shallow rooted small annual taxa (e.g. Centrolepis polyguna, Isolepts spp. and luncus bufonius) occupying the very surface lavers of the soil $(<1 \mathrm{~cm})$. Richness in the assemblage may be responding to local moisture availability or soil conditions not adequately reflected in our soil data. This may account for the low explanatory power of the model. Models for assemblages 10,17 and 19 had the greatest explanatory power $(72.4 \%, 73.0$ and $73.8 \%$ respectively). These assemblages had strong geographic patterning and climatic terms probably contributed to the explanatory power of the models.

Four assemblages (5,9,10 and 13) conformed to model assumptions and showed unimodal relationships between species composition and richness. These models however were only partially consistent with extrinsic knowledge of habitats and distributions. The edaphic model terms for assemblages 5, 9 and 13 were consistent with known habitat preferences, while the climate terms were partly artifacts of the location of the limited number of species rich quadrats in our data (assemblage 5), or the current study not sampling the assemblages major centre of distribution (assemblages 10 and 13).

A further seven assemblages $(2.3,2.6,4.2,8,11,15$ and 22) displayed an acceptable relationship between species richness and composition, however their richness data did not conform to the Poisson distribution. Assemblages 11 and 22 were regarded as artifacts. Three assemblages $(2.3,2.6$ and 4.2$)$ had low richness maxima and relatively few taxa. The explanatory power of these models was relatively low, although the model terms were generally consistent with known distributions and habitat preferences. Species rich occurrences of these assemblages were somewhat scattered and this may account for the low explanatory power of the models (i.e. poor correlation with regional climatic gradients). Models for assemblages 8 and 15 explained $67.5 \%$ and $66.9 \%$ of the total scaled deviance respectively, despite not conforming to model assumptions.

Assemblages 2.4 and 2.7 had very few taxa and showed little relationship between species richness and composition. Despite this, the component taxa were considered to form ecologically coherent assemblages, i.e. they contained taxa with similar habitat preferences, similar general distributions and were well sampled. Both assemblages occurred at quadrats that were species poor (assemblage 2.4, $10.2 \mathrm{taxa} /$ quadrat; assemblage 2.7, 13.15 taxa/ quadrat). The taxa within assemblage 2.4 occur in species poor stands with variable composition within southern sedge swamps and small freshwater bodies. No quadrats approached maximum assemblage richness. The model accounted for $66.3 \%$ of the variation in species richness (Table 9). Assemblage 2.7 also contained a collection of taxa regarded as ecologically meaningful. Again species richness and composition were not clearly related and model assumptions were not met, probably due to the small number of taxa.

For several assemblages the study area did not sample major parts of their known distributions. Coastal wetlands, particularly saline quadrats, were only sampled along the southern and northern coasts (i.e. models for assemblages 2.8, 3.3 and 7).

Some assemblages of essentially upland taxa were poorly sampled in the present dataset and were regarded as artifacts. Predictably, model terms were not consistent with the known distributions of these 
species (e.g. assemblage 11). In contrast, some terrestrial elements were well sampled in the context of their occurrence within wetland specific landforms such as lunettes. The model for assemblage 16 for example explained only $46.3 \%$ of the total scaled deviance and includes a group of species typical of mesic sandy lunettes, yet most taxa occur widely in non-wetland related landforms (see Gibson et al., 2004).

Elevation (ELCODE) was a significant term in 21 of the 29 assemblages for which models were constructed and 14 of the most robust 17 models. Clearly elevation is a surrogate for several other physical attributes we did not directly measure. Of primary importance is the frequency and duration of inundation. Elevation is also likely to be related to $\mathrm{EC}$, the degree of soil profile waterlogging and, particularly within large naturally saline systems, the level of soil profile development (berms versus old stabilized lunettes). Except for some ubiquitous annuals (e.g. elements of assemblage 18) no assemblages showed high richness at all elevations. These short-lived taxa with high powers of dispersal can utilize all elevations when conditions are favourable (e.g. drying lake floors).

EC and/or exchangeable sodium featured in 19 of the modeled groups and 15 of the 17 robust models (Table 8). The quadrat sampling captured the extreme ends of several environmental gradients, including fresh to highly saline substrates (soil EC ranged from $0.8 \mathrm{mS} \mathrm{m}^{-1}$ to $2980 \mathrm{mS} \mathrm{m}^{-1}$ ). Few assemblages occurred across a broad range of EC values (except assemblage 3.1).

Eleven models and seven of the most robust included $\mathrm{pH}$ as a significant term. For assemblages of naturally saline playas and pans, richness responses to $\mathrm{pH}$ could be related to assemblages of evaporites and soils derived from them, versus those of leached sand flats, sandy lunettes and granite derived soils. Other model variables were more assemblage specific. Gypsum was an explanatory variable for assemblage 2.6 and 3.3 ; and soil texture variables (percent sand, silt or clay) for assemblage $2.3,3.1,14,15$ and 16 (Table 8 ).

The most common climate terms in models were annual precipitation and annual temperature range. These terms highlight the strong geographic patterning of many assemblages (Figure 10).

\section{Singletons and rare species}

In the current survey, $31 \%$ of taxa were recorded from a single quadrat and $59 \%$ from less than five quadrats. In a survey of wetlands within the Carnarvon Basin Gibson et al. (2000) found $46 \%$ of taxa occurred in a single quadrat and $55 \%$ of taxa restricted to a single wetland. Similarly high numbers of singletons (ca. $33 \%$ ) were found by Halse et al. (2004) in a combined data matrix including plants (a subset of data from the current study), aquatic invertebrate and waterbird records from each of 197 wheatbelt and south coast wetlands. High numbers of rare species are also a common feature of quadrat based studies of nonwetland environments and have been widely reported in southwestern Australia (Gibson et al., 1994; Wardell-Johnson and Williams, 1996; Gibson et al., 2004).

Quadrats that showed high numbers of singletons were distributed at the periphery of the study area (Figure 4), particularly in the high rainfall south-west corner. A major biogeographical boundary (between the Jarrah Forest and Warren bioregions) occurs in this part of the study area. This boundary is more correctly described as a steep section of the compositional gradient (Gibson et al., 2004). The high number of rare taxa may relate to a phytogeographical edge effect in this area.

\section{Wetland plant biodiversity and secondary salinisation}

Occurring almost exclusively in the bottom of the landscape, wheatbelt wetlands and their riparian zones are regarded as being the communities most at risk from the impacts of dryland salinity (Cramer and Hobbs, 2002; Halse et al., 2003). An increase in shallow saline water tables, saline surface flows, soil profile waterlogging and altered flooding regimes will continue to have major impacts on wetlands and their associated vegetation. The impacts of altered hydrology on vegetation have been documented for some wheatbelt wetlands (Froend et al., 1987, Froend and McComb, 1991; Sanders, 1991, Halse et al., 1993).

Not all components of the wheatbelt wetland flora will be equally sensitive to secondary salinisation. In simple terms, plant species assemblages intolerant of flooding and/or increased root zone or water column salinity are likely to be threatened and tolerant assemblages will expand in spatial extent. Although we have limited detailed knowledge of the salinity tolerances of individual species, in broad terms assemblages associated with freshwater wetlands are likely to be those most at risk. In the current study five assemblages (2.1, 2.4, 3.1, 14 and 15) can readily be identified as being at risk of being lost from the study area. Collectively these assemblages contain 131 native taxa.

Assemblages 2.1 and 3.1 (41 native taxa) in particular were associated with quadrat groups 16 - northern claypan margins and basins, 17 margins of wooded freshwater swamps, 25 northern wooded freshwater basins and margins and 26 - Yate and Melaleuca strobophylla basins. These wetlands have already been recognized as being under immediate threat from salinisation. The Western Australian Department of 
Conservation and Land Management currently lists "Perched wetlands of the wheatbelt region with stands of living Swamp Sheoak (Casuarina obesa) and Paperbark (Melaleuca strobophylla) across the lake floor" as a critically endangered ecological community. Another, "Perched freshwater wetlands of the northern Wheatbelt dominated by extensive stands of living Eucalyptus camaldulensis (River Red Gum) across the lake floor" is also listed as a threatened ecological community. Morrila Swamp a Eucalyptus camaldulensis swamp in the northern wheatbelt was reported by George et al. (1995) as showing signs of stress and now the lakebed community is regarded as destroyed. Assemblages 2.4, 14 and 15 (90 taxa native) were associated with southwestern claypans, basins and riparian margins of non-saline wetlands (quadrat groups 19,20.1, 20.2 and 21) in the higher rainfall parts of the study area. The wetlands at which these assemblages occur are also being impacted by secondary salinisation (Keighery et al., 2002).

While the impacts of hydrological change on freshwater wetlands are primarily a matter of increasing salinity, likely scenarios for impacted naturally saline wetlands are more difficult to predict. The current study has highlighted the strong relationship between local elevation, substrate EC and $\mathrm{pH}$ and the floristic composition of the vegetation of naturally saline wetlands and their surrounds. Naturally saline wetlands of the study area will continue to experience greater inundation, through amplified flood events and greater groundwater discharge (George and Coleman, 2002). The shrublands surrounding these systems will be impacted when plant communities intolerant of flooding are inundated or when elevated groundwater levels lead to increased root zone salinity of more elevated vegetation. For much of the vegetation shallow saline water tables may act by preventing soil profile leaching by rainfall (Cramer and Hobbs, 2002)

Four assemblages $(3.2,4.1,4.2$, and 12) occur at the margins of naturally saline wetlands and are at particular risk from altered hydrology. In total they contain 60 native taxa. These assemblages were strongly associated with quadrat groups 8.2 and 9.2 and occurred at low elevations around the periphery of playas and saline pans. The substrates they occupy are relatively non-saline and they probably persist where lateral and vertical leaching of salts occurs. These assemblages are vulnerable to even small changes in wetland water depth and hydroperiod that might increase the risk of flooding and alter soil profile salinity.

Six assemblages $(16,17,18,19,20$ and 21) were associated with more elevated woodlands and shrublands within naturally saline wetlands. These plant communities are mostly elevated and unlikely to be directly inundated but are impacted by the loss of deep-rooted dominants such as Melaluaca and Eucalyptus due to increased root zone salinity. The native flora of these assemblages totaled 281 taxa. Even if localised site features protect some vegetation, it seems likely that even these more elevated wetland plant communities will continue to be fragmented and degraded.

In summary 15 plant assemblages of non-saline and naturally saline wetlands containing 472 native taxa are identified as being at particular risk from secondary salinisation. This represents a third of the taxa recorded during the current survey. Given the pervasiveness of the problem of dryland salinity in south-west Western Australia, the long-term conservation of these assemblages and their component species appears bleak.

\section{ACKNOWLEDGEMENTS}

We wish to thank the numerous private landholders who assisted in locating wetlands to sample and provided access to their land. P.G. Wilson, L. Craven, B.J. Lepschi, B.R. Maslin, B.L. Rye, K.A. Sheppard and M.E. Trudgen assisted in the identification of specimens and provided information on their distributions. J.K. Rolfe assisted with multivariate analyses. P. Gioia ran the ANUCLIM software package to generate climate attributes. D. Allen and R. Harper provided advice on the interpretation of soils data and the Western Australian Chemistry Centre (chemical) and the Department of Conservation and Land Management Science Division soil laboratory (mechanical) conducted the soils analyses. Funding for this study was provided as part of the State Salinity Strategy.

\section{REFERENCES}

Anonymous (1996). Western Australian salinity action plan. Government of Western Australia, Perth.

Atkins, K.J. (2003). Declared rare and priority flora list for Western Australia. Department of Conservation and Land Management, Perth.

Austin, M.P. and Belbin, L. (1982). A new approach to the species classification problem in floristic analysis. Australian foumal of Ecology 7: 75-89.

Barnsley, B. (1982). Frankeniaceae In A.S. George (ed), Flora of Australin, wol. 8. Lecythidales to Batales: 112-146. Bureau of Flora and Fauna, Canberra.

Beard, J.S. (1980). A new phytogeographic map of Western Australia. Western Australian Herbarium Reserarch Notes 3: 37-58

Beard, J.S. (1982). Late Pleistocene aridity and aeolian landforms in Western Australia. In W.R. Barker and P.J.M. Greenslade (eds), Evolution of the flora and fanm of arid Australia: 101-106. Peacock Publications, South Australia.

Beard, JS (1999). Evolution of the river systems of the 
South-west drainage division, Western Australia. Journal of the Royal Society of Western Australia 82: 147 164.

Beard. J.S. (2000). Drainage evolution in the Moore Monger system, Western Australia. Journal of the Royal Society of Western Australia 83: 29-38.

Beard, J.S., Chapman, A.R. and Gioia P. (2000). Species richness and endemism in the Western Australian flora. Journal of Biogeography 27: 1257-1268.

Belbin, L. (1995). PATN technical reference. CSIRO, Canberra.

Bettenay, E. (1962) The salt lake systems and their associated aeolian features in the semi-arid regions of Western Australia. Journal of Soil Science 13: 10-18.

Beresford, Q., Bekle, H., Philips, H. and Mulcock, J. (2001). The salinity crisis: landscapes, communities and politics. University of Western Australia Press, Perth.

Bowler, J.M. (1983). Lunettes as indices of environmental change: a review of Australian evidence. Proceedings of the Royal Society of Victoria 95: 147-168.

Brock, M.A. and Lane, J.A.K. (1983). The aquatic macrophyte flora of saline wetlands in Western Australia in relation to salinity and permanence. Hydrobiologia 105: 63-76.

Burbidge, A.H., Harvey, M.S. and McKenzie, N.L. (eds). (2000). Biodiversity of the southern Carnarvon Basin. Records of the Western Australian Museum Supplement 61.

Czekanowski, J. (1909). Zur Differentialdiagnose der Neandertalgruppe. Korrespondenz-Blatt der deutschen Gesellschaft für Anthropologie, Ethnologie und Urgeschichte 40: 44-47.

Cope, R.N. (1975). Tertiary epeirogeny in the southern part of Western Australia. Annual Report 1974: 40-46. Geological Survey of Western Australia, Perth.

Cramer, V.A. and Hobbs, R.J. (2002). Ecological consequences of altered hydrological regimes in fragmented ecosystems in southern Australia: impacts and possible management responses. Austral Ecology 27: 546-564.

Craven, L.A., Lepsch,i B.J., Broadhurst, L. and Byrne M. (2004). Taxonomic revision of the broombush complex in Western Australia (Myrtaceae, Melaleuca uncinata s.1.). Australian Systematic Botany 17: 255-271.

Dufrene, M. and Legendre, P. (1997). Species assemblages and indicator species: the need for a flexible asymmetrical approach. Ecological Monographs 67 : 345-366.

Environment Australia (2000). Revision of the interim biogeographic regionalisation of Australia (IBRA) and development of version 5.1. Environment Australia, Canberra.

Froend, R.H. and van der Moezel, P.G. (1994) The impact of prolonged flooding on the vegetation of Coomalbidgup Swamp, Western Australia. Journal of the Royal Society of Western Australian 77: 15-22.

Froend, R.H., Heddle, E.M., Bell, D.T. and McComb, A.J. (1987). Effects of salinity and waterlogging on the vegetation of Lake Toolibin, Western Australia. Australian Journal of Ecology 12: 281-298.

Froend, R.H. and McComb, A.J. (1991). An account of the decline of Lake Towerrining, a wheatbelt wetland.
Journal of the Royal Society of Western Australia. 73: 123128.

George, R.J., McFarlane, D.J. and Speed, R.J. (1995). The consequences of changing hydrologic environment for native vegetation in Western Australia. In D.A. Saunders, J.L. Craig and E.M. Mattiske (eds), The role of networks: 9-22. Surrey Beatty \& Sons, Sydney.

George, R.J. and Coleman, M. (2002). Hidden menace or opportunity - groundwater hydrology, playas, and commercial options for salinity in wheatbelt valleys. In V. Read \& Associates (eds), Dealing with salinity in wheatbelt valleys: processes, prospects and practical options: 1-21. Waters and Rivers Commission Perth. (Available on CD).

Gibson, N., Keighery, B.J., Keighery, G.J., Burbidge, A.H. and Lyons, M.N. (1994). A floristic survey of the southern Swan Coastal Plain. Unpublished report. Department of Conservation and Land Management, Perth.

Gibson, N. Keighery, G.J. and Lyons, M. N. (2000). The flora and vegetation of the seasonal and perennial wetlands of the southern Carnarvon Basin, Western Australia. Records of the Western Australian Museum Supplement 61: 175-199.

Gibson, N. and Keighery, G.J. (2000) Flora and Vegetation of the Byenup - Muir reserve system, south-western Western Australia. CALMScience 3: 323-402.

Gibson, N, Keighery, G.J, Lyons, M.N. and Webb, A. (2004) The terrestrial flora and vegetation of the Western Australian wheatbelt. Records of the Western Australian Museum Supplement 67: 139-189.

Halse, S.A., Pearson, G.B. and Patrick, S. (1993). Vegetation of depth-gauged wetlands in nature reserves of the south-west Western Australia. Technical Report 30. Department of Conservation and Land Management, Perth.

Halse, S.A., Ruprecht, J.K. and Pinder, A.M. (2003). Salinisation and prospects for biodiversity in rivers and wetlands of south-west Western Australia. Australian Journal of Botany 51: 673-688.

Halse, S.A., Lyons, M.N., Pinder, A.M. and Shiel, R.J. (2004) Biodiversity patterns and their conservation in wetlands of the Western Australian wheatbelt. Records of the Western Australian Museum Supplement 67: 337-364.

Hatton, T. and Ruprecht, J. (2002). Watching the rivers flow: hydrology of the wheatbelt. In V. Read \& Associates (eds), Dealing with salinity in Wheatbelt Valleys: processes, prospects and practical options: 1-15. Waters and Rivers Commission, Perth. (Available on CD).

Hickman, J.C. (ed) (1993). The Jepson manual of higher plants of California. University of California Press, Berkeley.

Hobbs, R.J., Saunders, D.A., Lobry de Bruyn, L.A. and Main, A.R. (1993). Changes in biota. In R.J. Hobbs and D.A. Saunders (eds), Reintergrating fragmented landscapes: towards sustainable production and nature conservation: 65-106. Springer-Verlag, New York.

Hopper, S.D., Harvey, M.S., Chappill, J.A., Main, A.R. and Main B.Y. (1996) The Western Australian biota as Gondwanan heritage - a review. In S.D. Hopper, J.A. 
Chappill, M.S. Harvey and A.S. George (eds), Gondzanan heritage: past, present, and future of the Western Australian biota: 1-46. Surrey Beatty \& Sons, Sydney.

Houlder, D.J., Hutchinson, M.F., Nix, H. A. and McMahon, J.P. (1999). ANUCLIM user guide, version 5.0. Centre for Resource and Environmental Studies, Australian National University, Canberra.

Jacobs, S.W.L. and Brock, M. (1982). A revision of the genus Ruppia (Potamogetonaceae) in Australia. Aquatic Botany 14: 325-337.

Jacobs, S.W.L. and Wilson, K.L. (1996). A biogeographical analysis of the freshwater plants of Australasia. Australian Systematic Botany 9: 169-183.

Jones, D.C. (1994). Gypsum deposits of Western Australia. Record 1993/5. Geological Survey of Western Australia, Perth.

Keeley, J.E. and Zedler, P.H. (1998). Characterization and global distribution of vernal pools. In C.W. Witham, E.T. Bauder, D. Belk, W.R. Ferren and R. Ornduff (eds), Ecology, conserontion, and management of vernal pool ecosystems. Procedings from a 1996 conference: 114. California Native Plant Society, Sacramento CA.

Keighery, G.J., Gibson, N., Lyons, M.N. and Burbidge, A.H. (2000). Flora and vegetation of the southern Camarvon Basin, Western Australia. Records of the Western Australinn Museum 61: 77-154.

Keighery, G.J., Gibson, N., Webb, A.. and Muir, W.P (2002). A biological survey of the agricultural zone vegetation and flora of Drummond Nature Reserve. Conservation Science Western Australia 4: 63-78.

Keighery, G.J. and Keighery, B.J. (2003). Juncaginaceae in Western Australia. Centre of species diversity of another family in this mega-diverse region. Conference abstract. Monocots III. Third International Moncots Conference, Rancho Santa Ana Botanic Garden, Claremont, California.

Keighery, G.J. (in press a). Taxonomic notes on the Angianthus drummondii complex (Asteraceae: Gnaphalinae). Nuytsin.

Keighery, G.J. (in press b) Taxonomic notes on Actinotus, Apilim, and Erymgium (Apiaceae) from Western Australia. Nuytsin.

Kühn, U., Bittrich, V., Carolin, R., Reitag, H., Hedge, I.C., Uotila P. and Wilson, P. (1993). Chenopodiaceae. In K. Kubitzki, J. G. Rohwer, and V. Bittrich (eds), The fanilies and genera of vascular plants. Flowering plants dicotyledons: 253-280. Springer-Verlag, Berlin.

Lane, J.A.K and McComb, A.J. (1988). Western Australian wetlands. In A.J. McComb and P.S. Lake (eds), The conservation of Australian wetlands: 127-146. Surrey Beatty \& Sons, Sydney.

Michael, P.W. (1999). Eryngium. In N.G. Walsh and T.J Entwisle (eds), Flora of Victoria vol 4: 260-263. Inkata Press, Melbourne.

Mckenzie, N.L. Gibson, N., Keighery, G.J. and Rolfe, J.K. (2004) Patterns in the terrestrial environments of the Western Australian wheatbelt. Records of the Western Australian Museum Supplement 67: 293-335
Mulcahy, M.J. (1967). Landscapes, laterites and soils in southwestern Australia. In J.N. Jennings and J.A. Mabbutt (eds), Landform studies from Australia and New Guinea: 211-230. Australian National University, Canberra.

Myers, N., Mittermeler, C.G., da Fonseca, G.A.B. and Kent, 1. (2000). Biodiversity hotspots for conservation priorities Nature 403: 853-858.

NAG (1986). The generalized linear interactive modeling system. Release 4.0 manual. Numerical Algorithms Group, Oxford.

Public Works Department (1984). Streamflow records of Western Australia to 1982. Volume 2: Basins 613-617. Public Works Department, Western Australia,

Sanders, A. (1991). Oral histories documenting changes in wheatbelt wetlands. Occasional Paper $2 / 91$. Department of Conservation and Land Management, Perth.

Schofield, N.J., Ruprecht, J.K. and Loh, I.C. (1988). The impact of agricultural development on the salinity of surface waters resources of the south-west of Western Australia. Report WS 27. Water Authority of Western Australia, Perth.

Sheppard, D.P. Beeston, G.R. and Hopkins, A.J.M. (2002). Native vegetation in Western Australia: extent, type and status. Resource Management Technical Report Department of Agriculture, Perth..

Short, P.S. (1983). A revision of Angianthus Wendl., sensu lato (Compositae: Inuleae: Gnaphaliinae). Muelleria 5 $143-214$.

Short, R.J. and McConnell, C. (2001). Extent and impacts of dryland salinity. Resource Management Technical Report 202. Department of Agriculture, Perth.

Sneath, P.H.A. and Sokal, R.R. (1973). Numerical taxonomy. The principles and practice of numerical classification. W.H. Freeman, San Francisco.

Teakle, L.J.H. and Burvill, G.H. (1938). The movement of soluble salts in soils under light rainfall conditions. Journal of Agriculture of Western Australia 15: 218-245.

Thackaway R. and Cresswell, I.D. (eds) (1995) An interim biogeographic regionalisation for Australia: a framework for setting priorities in the National Reserves System Cooperative Program, version 4.0. Australian Nature Conservation Agency, Canberra

Wardell-Johnson, G.W. and Williams, M. (1996). A floristic survey of the Tingle mosaic, south-western Australia: applications in land use planning and management. Journal of the Royal Society of Western Australia 79: 241-276.

Watson, L. and Dallwitz, M.J. (2000). The families of flowering plants: descriptions, illustrations, identification, and information retrieval, version 14 th December 2000. http//biodiversity uno.edu/delta/

Wilson, P.G. (1980). A revision of the Australian species of Salicomieae (Chenopodiaceae). Nuytsia 3: 3-154.

Wood, W.E. (1924). Increase of salt in soil and streams following the destruction of native vegetation. Jourmal of the Royal Society of Western Australia 10: $35-48$.

\section{Electronic appendices are on $C D$ inside the back cover}

